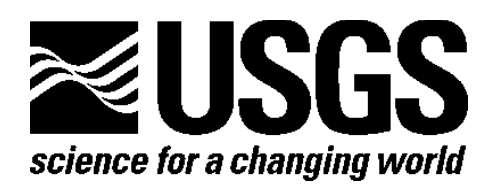

\title{
Coalbed Natural Gas Exploration, Drilling Activities, and Geologic Test Results, 2007-2010, Wainwright, Alaska
}

By Arthur C. Clark

Open-File Report 2014-1004

U.S. Department of the Interior

U.S. Geological Survey 


\section{U.S. Department of the Interior \\ SALLY JEWELL, Secretary}

\section{U.S. Geological Survey \\ Suzette M. Kimball, Acting Director}

U.S. Geological Survey, Reston, Virginia: 2014

For more information on the USGS—the Federal source for science about the Earth, its natural and living resources, natural hazards, and the environment-visit http://www.usgs.gov or call 1-888-ASK-USGS

For an overview of USGS information products, including maps, imagery, and publications, visit $h$ ttp://www.usgs.gov/pubprod

To order this and other USGS information products, visit $h$ ttp://store.usgs.gov

Suggested citation:

Clark, A.C., 2014, Coalbed natural gas exploration, drilling activities, and geologic test results, 2007-2010, Wainwright, Alaska: U.S. Geological Survey Open-File Report 2014-1004, 65 p., http://dx.doi.org/10.3133/ofr20141004.

ISSN 2331-1258 (online)

Any use of trade, firm, or product names is for descriptive purposes only and does not imply endorsement by the U.S. Government.

Although this information product, for the most part, is in the public domain, it also may contain copyrighted materials as noted in the text. Permission to reproduce copyrighted items must be secured from the copyright owner. 


\section{Contents}

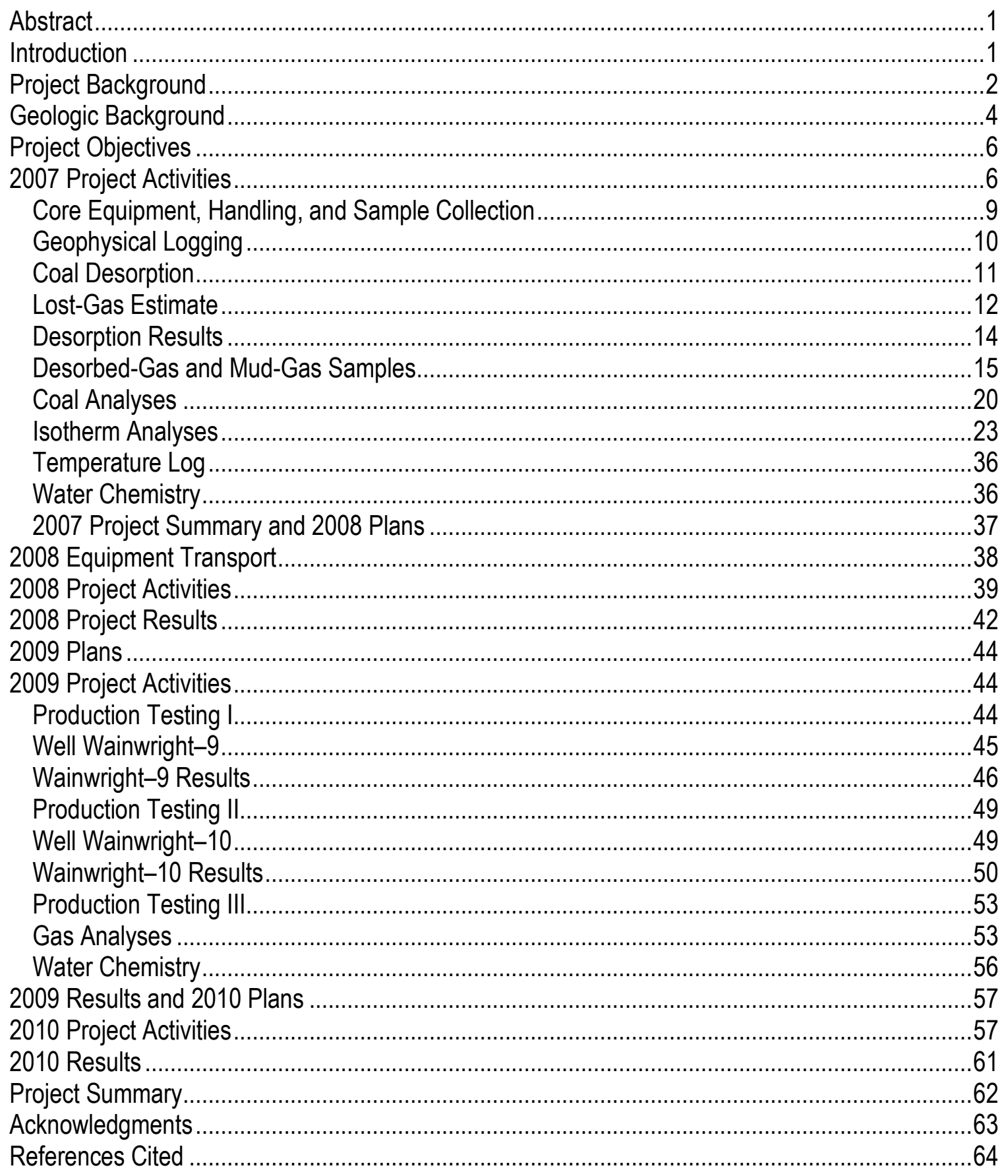

\section{Figures}

Figure 1. Map of Alaska showing location of Alaska Rural Energy Project coalbed natural gas study areas in green 
Figure 2. Map showing location of drill holes, seismic lines, and coal isopach used to determine approximate depth of coal-bearing strata and net thickness of coalbeds beneath Wainwright

Figure 3. Aerial photograph of Wainwright, Alaska, and the location of site

Wainwright-1.....

Figure 4. Alaska Rural Energy Project rig drilling the Wainwright-1 coalbed natural gas exploration core hole.

Figure 5. Project geologists describing and boxing core samples from the

Wainwright-1 core hole.

Figure 6. Wainwright-1 coal desorption canisters in thermal baths ........................................12

Figure 7. Graph depicting desorption regression line used to determine lost-gas value. ...........13

Figure 8. Isotherm chart from well Wainwright-1, desorption canister W1-4, analyzed

by Ticora Laboratories

Figure 9. Isotherm chart from well Wainwright-1, desorption canister W1-9, analyzed

by Ticora Laboratories

Figure 10. Isotherm chart from well Wainwright-1, desorption canister W1-11, analyzed

by Ticora Laboratories

Figure 11. Isotherm chart from well Wainwright-1, desorption canister W1-15, analyzed

by Ticora Laboratories

Figure 12. Isotherm chart from well Wainwright-1, desorption canister W1-18, analyzed

by Ticora Laboratories

Figure 13. Isotherm chart from well Wainwright-1, desorption canister W1-19, analyzed

by Ticora Laboratories.

Figure 14. Isotherm chart from well Wainwright-1, desorption canister W1-19, analyzed

by RMB Earth Science Consultants, Inc.

Figure 15. Isotherm chart from well Wainwright-1, desorption canister W1-21, analyzed

by Ticora Laboratories

Figure 16. Isotherm chart from well Wainwright-1, desorption canister W1-21, analyzed

by RMB Earth Science Consultants, Inc.

Figure 17. Isotherm chart from well Wainwright-1, desorption canister W1-23, analyzed

by Ticora Laboratories

Figure 18. Isotherm chart from well Wainwright-1, desorption canister W1-25, analyzed

by Ticora Laboratories

Figure 19. Wainwright-2 temperature log collected by the University of Alaska Fairbanks......36

Figure 20. Diagram of proposed Wainwright Olgoonik Corporation ware-yard

production-test-well array......

Figure 21. Unloading truck-mounted drill rig from C-130 transport plane in

Wainwright, Alaska.

Figure 22. Wainwright Olgoonik Corporation ware-yard production-test-well array

Figure 23. Installing the variable-frequency-drive pump control for production testing

of the W-OCP (Wainwright-3) well.

Figure 24. Fluid and ice produced from submersible-pump discharge pipe during initial 2009 production testing in Wainwright well W-OCP.

Figure 25. Drilling the Wainwright-10 delineation well

Figure 26. Surface-drive head assembly installed on W-OCP production well.......................59

Figure 27. Caliper log collected from uncased portion of the W-OCP well on July $29,2010 \ldots .61$ 


\section{Tables}

Table 1. Summary of Wainwright-1 canister desorption results............................................15

Table 2. Wainwright-1 desorbed-gas compositional analysis ..............................................17

Table 3. Wainwright-1 as-received desorbed-gas isotopic analyses ....................................18

Table 4. Wainwright-1 mud-gas analysis .......................................................................19

Table 5. As-received geochemical analysis of well Wainwright-1 desorbed-coal samples.......21

Table 6. Mud-gas analysis from Wainwright well W-OC1 (Wainwright-8)................................43

Table 7. Raw desorption results from Wainwright-9 coal core samples .................................46

Table 8. As-received geochemical analysis of well Wainwright-9 desorbed-coal samples.......47

Table 9. Dry-ash-free (DAF) desorption results from two Wainwright-9 coal samples .............48

Table 10. Raw desorption results from Wainwright-10 coal core samples ..............................51

Table 11. As-received geochemical analysis for two Wainwright-10 desorbed-coal samples ....51

Table 12. Dry-ash-free (DAF) desorption results from two Wainwright-10 coal samples............52

Table 13. Compositional analysis of gas samples collected during 2009 season .......................55

Table 14. As-received isotopic analyses of gas samples collected during 2009 season.............56

Table 15. Chemical analysis of water samples collected from a sandstone in well

Wainwright-10 and the Wainwright coalbed in well W-OCP ...................................................56

\section{Plates}

Plate 1. Slabbed core photos ..................................................................................... link

Plate 2. Geophysical logs from wells Wainwright -1 and Wainwright-2 .................................... link 


\section{Initialisms}

AOGCC Alaska Oil and Gas Conservation Commission

AREP Alaska Rural Energy Project

ASRC Arctic Slope Regional Corporation

ASTM American Society for Testing and Materials

BLM Bureau of Land Management

CBNG coalbed natural gas

DAF dry-ash-free

DGGS Department of Geological and Geophysical Surveys

DOE U.S. Department of Energy

NSB North Slope Borough

OC Olgoonik Corporation

PC progressive cavity

PVC polyvinyl chloride

STP standard temperature and pressure

TDS total dissolved solids

UAF University of Alaska Fairbanks

USGS U.S. Geological Survey 


\title{
Coalbed Natural Gas Exploration, Drilling Activities, and Geologic Test Results, 2007-2010, Wainwright, Alaska
}

\author{
By Arthur C. Clark
}

\section{Abstract}

The U.S. Geological Survey, in partnership with the U.S. Bureau of Land Management, the North Slope Borough, and the Arctic Slope Regional Corporation conducted a four-year study designed to identify, define, and delineate a shallow coalbed natural gas $(\mathrm{CBNG})$ resource with the potential to provide locally produced, affordable power to the community of Wainwright, Alaska. From 2007 through 2010, drilling and testing activities conducted at three sites in or near Wainwright, identified and evaluated an approximately 7.5 -ft-thick, laterally continuous coalbed that contained significant quantities of CBNG. This coalbed, subsequently named the Wainwright coalbed, was penetrated at depths ranging from 1,167 ft to 1,300 $\mathrm{ft}$ below land surface. Core samples were collected from the Wainwright coalbed at all three drill locations and desorbed-gas measurements were taken from seventeen 1-ft-thick sections of the core. These measurements indicate that the Wainwright coalbed contains enough CBNG to serve as a long-term energy supply for the community.

Although attempts to produce viable quantities of CBNG from the Wainwright coalbed proved unsuccessful, it seems likely that with proper well-field design and by utilizing currently available drilling and reservoir stimulation techniques, this CBNG resource could be developed as a long-term economically viable energy source for Wainwright.

\section{Introduction}

Alaska has an essential need to identify and develop local, clean energy alternatives for remote communities that currently rely on diesel fuel for power generation. Alaska's North Slope is underlain by vast coal deposits estimated to hold between 7 and 36 trillion cubic feet (TCF) of coalbed natural gas (CBNG) according to a U.S. Geological Survey (USGS) assessment of North Slope coalbed natural gas resources (Roberts and others, 2008). This widespread resource has the potential to serve as a longterm, locally produced energy supply for North Slope communities that overlie coal deposits. Geologic and petrophysical evaluation of CBNG is needed to determine production capability and viability in a given location. Analyses of parameters such as depth to coal, coalbed thickness, coal rank, gas content, reservoir storage capacity, depth of permafrost, and reservoir pressure and permeability are needed to provide an accurate 
assessment. These critical data can only be collected through exploratory drilling, testing, and analytical procedures.

The U.S. Department of the Interior Alaska Rural Energy Project (AREP) is a collaborative research project between the USGS and the Alaska Bureau of Land Management (BLM) that is tasked to identify and evaluate shallow subsurface resources, such as CBNG, as a power alternative in remote Alaskan communities. A 4-year study was conducted in the North Slope community of Wainwright, Alaska, a Native community of approximately 600 people located next to the Chukchi Sea $70 \mathrm{mi}$ southwest of Barrow (fig. 1). The AREP, in partnership with the North Slope Borough (NSB) and the Arctic Slope Regional Corporation (ASRC), conducted drilling and testing activities designed to identify, define, and delineate a shallow CBNG resource with the potential to provide locally produced, affordable power to the community.

A 1,613-ft-deep core hole drilled in Wainwright in 2007 penetrated numerous coalbeds, ranging in thickness from several inches to $7.6 \mathrm{ft}$, nearly all of which contained significant levels of methane gas. Although most beds were located within the approximately 1,000-ft-thick zone of permafrost, a 7.6-ft-thick coalbed was encountered below the permafrost at a depth of 1,242 ft. This bed, informally named the Wainwright coalbed, became the target of subsequent delineation drilling and production testing activities conducted during the summers of 2008, 2009, and 2010. This report provides a project timeline, describes activities and procedures, presents geologic data, and provides gas-desorption, isotherm, and chemical test results from the 4-year study.

\section{Project Background}

As summarized by Tyler and others (2000), the Colville (North Slope), Yukon Flats, and Alaska Peninsula Basins (fig. 1), in descending order, have been identified as having the greatest $\mathrm{CBNG}$ development potential for areas in which primary off-grid Alaskan communities are located. In 2000, the U.S. Department of Energy (DOE) funded the Alaska Department of Geological and Geophysical Surveys (DGGS) and the USGS to conduct field studies in these basins near the communities of Wainwright, Fort Yukon, and Chignik, Alaska (fig. 1), in advance of possible CBNG drilling and testing activities near these villages. After completing these studies, the Alaska BLM and the USGS formed the AREP and, in 2004, purchased a portable diamond-core drilling rig to conduct shallow CBNG and other alternative energy drilling and testing activities near remote Alaskan communities. In 2004, the AREP, with primary funding from DOE and in collaboration with the DGGS, conducted exploratory CBNG drilling and testing activities at Fort Yukon (for results, see Clark and others, 2009). Another test hole was drilled in the permafrost on Alaska's North Slope near Franklin Bluffs in 2005. The equipment was stored in Deadhorse, Alaska (fig. 1), and in the summer of 2006 was barged from Prudhoe Bay to Wainwright where exploratory CBNG drilling and testing activities began in the summer of 2007. Primary funding for the 2007 activities was furnished by BLM with supplemental funding provided by USGS and ASRC. 


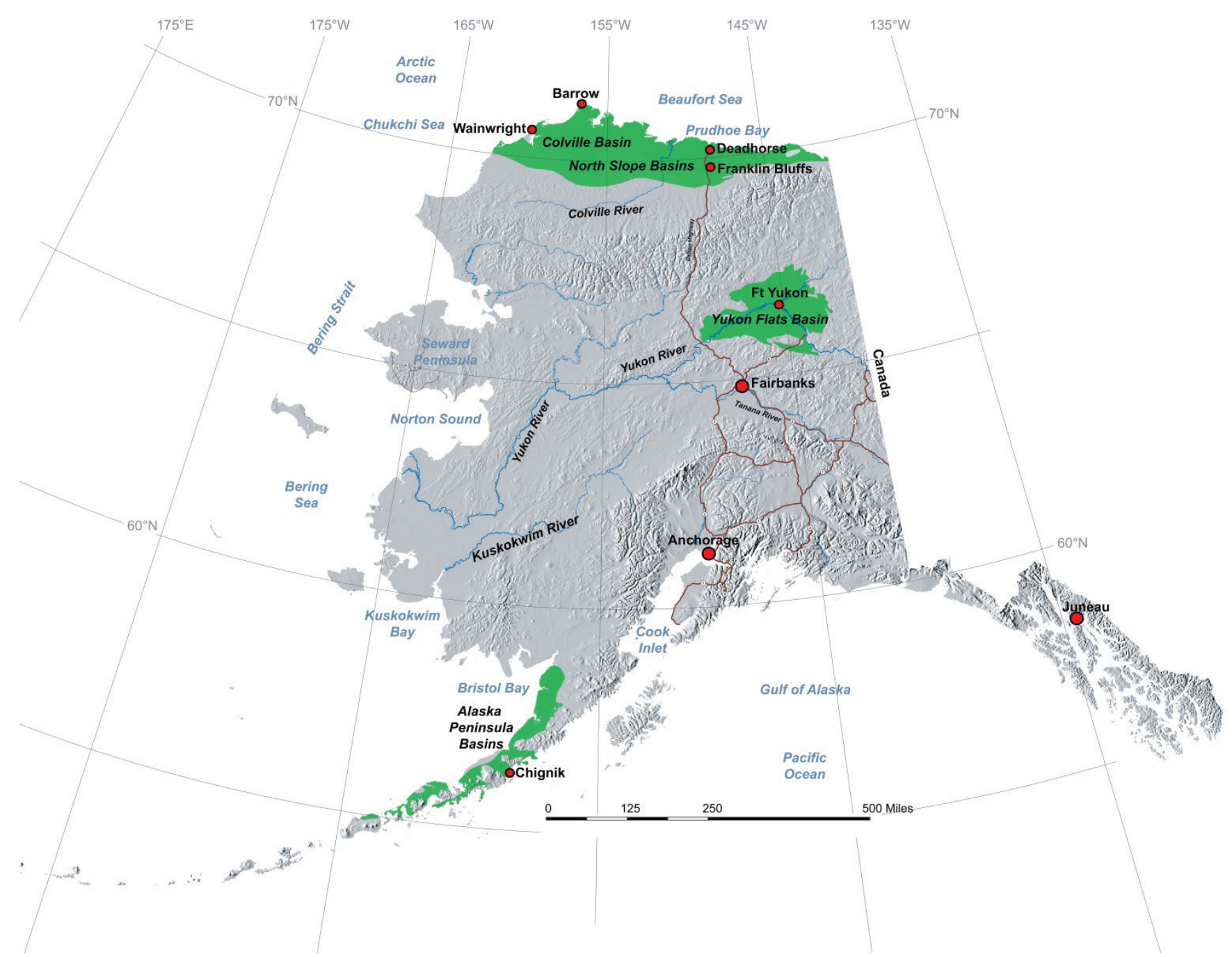

Figure 1. Map of Alaska showing location of Alaska Rural Energy Project coalbed natural gas study areas in green. 


\section{Geologic Background}

The village of Wainwright sits atop a nonmarine sedimentary facies of the Cretaceous (lower Albian to Cenomanian) Nanushuk Formation (Mull and others, 2003; Ahlbrandt and others, 1979), which contains up to 150 bituminous and subbituminous coalbeds ranging in thickness from a few inches to $20.2 \mathrm{ft}$ (Flores and others, 2004). In the western part of the North Slope, the Nanushuk Formation includes the interval that was formerly designated the Corwin and Kukpowruk Formations (now abandoned) of the Nanushuk Group (see Molenaar1985; Wahrhaftig and others, 1994) and is composed of a thick deltaic sequence of intertonguing marine and nonmarine deposits (Molenaar, 1985). The upper nonmarine unit, primarily composed of coal-bearing delta- and alluvial-plain deposits (Chapman and Sable, 1960; Ahlbrandt and others, 1979), and the lower marine unit, consisting of offshore sands, silts, and shale (Molenaar, 1985), is separated by an intertonguing transitional unit that also contains coal deposits (Molenaar, 1985; Ahlbrandt and others, 1979; Moore and others, 1994; Callahan and Sloan, 1978; Wahrhaftig and others, 1994).

Geophysical log data from three oil and gas wells drilled in the general vicinity of Wainwright, Tunalik-1 (lat $70^{\circ} 12^{\prime} 21.45^{\prime \prime} \mathrm{N}$., long $161^{\circ} 05^{\prime} 09.16^{\prime \prime} \mathrm{W}$.), Kugrua-1 (lat $70^{\circ} 35^{\prime} 13.28^{\prime \prime} \mathrm{N}$., long $158^{\circ} 39^{\prime} 43.25^{\prime \prime} \mathrm{W}$.), and Peard-1 (lat 70 $42^{\prime} 56.32^{\prime \prime} \mathrm{N}$., long $159^{\circ} 00^{\prime} 02.52^{\prime \prime} \mathrm{W}$.) (fig. 2) were combined with data from two seismic lines (WM-WM', $\mathrm{TP}-\mathrm{TP}^{\prime}$ ) near Wainwright (fig. 2). These data indicate that coal-bearing strata exists beneath the community to a depth of between 1,500 and 2,000 ft before grading into the lower noncoal-bearing part of the Nanushuk Formation and marine-dominated sediments of the underlying Torok Formation (Molenaar, 1985; Sable and Stricker, 1987). USGS coal isopach maps (Roberts, 2008) based on these and other oil and gas logs suggest that between 50 and $100 \mathrm{ft}$ of net coal lies beneath Wainwright (fig. 2) and mud-gas logs from the three oil and gas wells indicate that coalbeds, both in and below the permafrost zone, likely contain methane gas. Although cross sections show that much of the coal lies within the permafrost zone, it also appeared likely that coal-bearing strata extended into the subpermafrost as well. Data contained within USGS borehole temperature logs from Arctic Alaska pre-1989 database (http://esp.cr.usgs.gov/data/bht_alaska/) indicate that the zone of permafrost, based on a $0{ }^{\circ} \mathrm{C}$ isotherm, extends to a depth of approximately $1,000 \mathrm{ft}$ beneath the Wainwright community. 


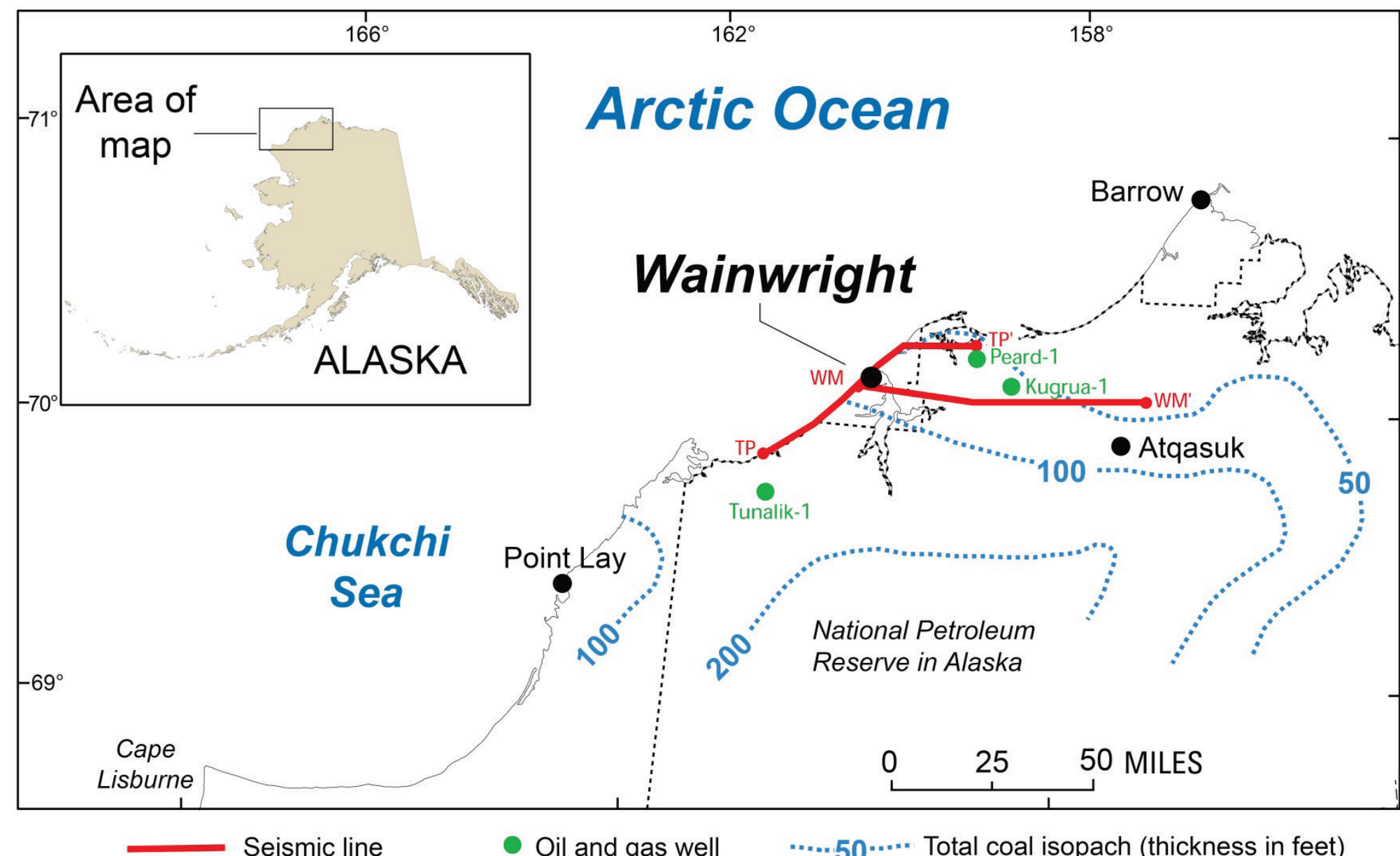

Figure 2. Map showing location of drill holes, seismic lines, and coal isopach used to determine approximate depth of coal-bearing strata and net thickness of coalbeds beneath Wainwright (modified from Roberts, 2008). 


\section{Project Objectives}

Based on the available data, initial project plans were to drill a continuous core hole near Wainwright to a depth of between 2,000 and 2,500 ft that would allow AREP to conduct a preliminary evaluation of local CBNG occurrence. Priority 2007 objectives included the following:

1. Determining the depth to, and thickness of, individual coalbeds.

2. Determining the sorbed-gas content of coal cores using canister desorption techniques.

3. Collecting borehole geophysical logs.

4. Collecting hydraulic data from significant ( $>5 \mathrm{ft}$ thick) subpermafrost coalbeds.

5. Collecting desorbed-gas samples for compositional analyses.

6. Determining gas storage capacity of tested coalbeds at reservoir temperature and pressure.

7. Determining geochemical composition and rank of coalbeds.

8. Collecting borehole temperature data to determine approximate permafrost thickness.

Based on the evaluation of these data, the AREP and project partners would determine if further CBNG investigations in the Wainwright vicinity were warranted.

\section{Project Activities}

Due to the spongy tundra that encompasses Wainwright and vicinity, there are few locations that provide the access and space required to conduct summer drilling and testing activities. Notwithstanding, the decision was made to conduct project operations during the summer months due to the increased difficulties and costs incurred when operating in arctic winter conditions. After previewing the area in 2006, a suitable site was chosen in the Olgoonik Corporation (OC) ware-yard on the northeast edge of the community. An estimated 2,500-ft well was permitted through the Alaska Oil and Gas Conservation Commission (AOGCC) and designated as Wainwright-1 (lat 70 $38^{\prime}$ $41.0^{\prime \prime} \mathrm{N}$., long $160^{\circ} 01^{\prime} 18.7^{\prime \prime} \mathrm{W}$.) (fig. 3). 


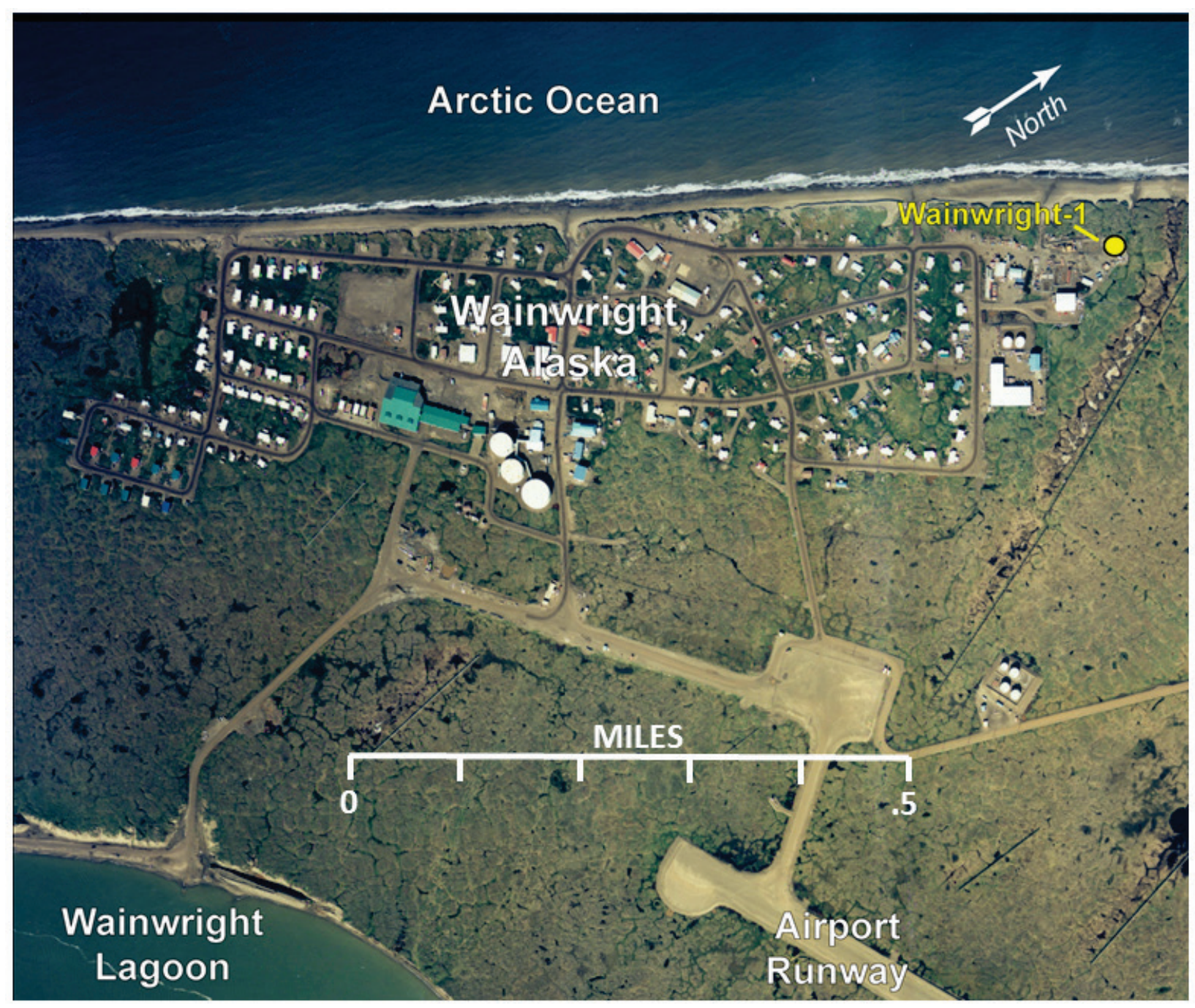

Figure 3. Aerial photograph of Wainwright, Alaska, and the location of site Wainwright-1. 
After arriving in Wainwright on June 8, 2007, with additional equipment and supplies flown in from Deadhorse via C-130 air transport, snow was removed from around the over-wintered equipment, and the site was prepared for drilling operations. Drilling began on June 11 (fig. 4) and 6-in. diameter surface casing was set and cemented to a depth of $75 \mathrm{ft}$. Coring operations began on June 14 and continued without incident through beds of siltstone, sandstone, mudstone, and coal to a depth of 1,605 ft (slabbedcore photos shown in plate 1). On June 27, while drilling at 1,613 ft, fluid circulation to the core bit was accidently interrupted, which caused the bit to become stuck in the bottom of the hole and the drill rods to fracture. Subsequent retrieval operations recovered the rods to a depth of $957 \mathrm{ft}$, but after additional unsuccessful recovery attempts, it was concluded that the remaining rods were irretrievably stuck. While collecting geophysical logs through the open portion of the well, it was discovered that the logging tools could be lowered through the top of the broken rods and run through the rods to the bottom of the hole. Logs that could be collected through the steel rods (natural gamma and density) were subsequently run from 1,613 to $957 \mathrm{ft}$, and a full set of logs was collected from $957 \mathrm{ft}$ to land surface. On July 5, after a failed attempt to deflect past the broken rods and drill a parallel bore to a greater depth, a 2.5-in.-diameter temperature-monitor casing was set and grouted to a depth of $956 \mathrm{ft}$ for the University of Alaska Fairbanks (UAF) and filled with a propylene glycol/water antifreeze mixture. The drill rig was then moved west approximately $25 \mathrm{ft}$ to drill a second well from which to collect hydraulic data from a 7.6-ft-thick coalbed, informally named the Wainwright coalbed, penetrated in Wainwright-1 at a depth of 1,242 ft. An open-hole bore, subsequently permitted as Wainwright-2 (lat $70^{\circ} 38^{\prime} 41.0^{\prime \prime} \mathrm{N}$., long $160^{\circ} 01^{\prime} 19.3^{\prime \prime} \mathrm{W}$.), was drilled to a depth of 1,295 ft between July 7 and 11 . Geophysical logs were collected on July 12, and the Wainwright coalbed was isolated with a set of pneumatic packers. Hydraulic data and water samples were collected from the Wainwright bed on July 12 and 13. After deflating and removing the packer string, a second temperature-monitor casing was set, grouted, and filled with antifreeze to a depth of 1,260 ft on July 14. Core samples were flown from Wainwright to Anchorage on a DC-6 charter on July 14, and the remaining equipment was winterized and stored in the OC yard for possible re-use in 2008. 


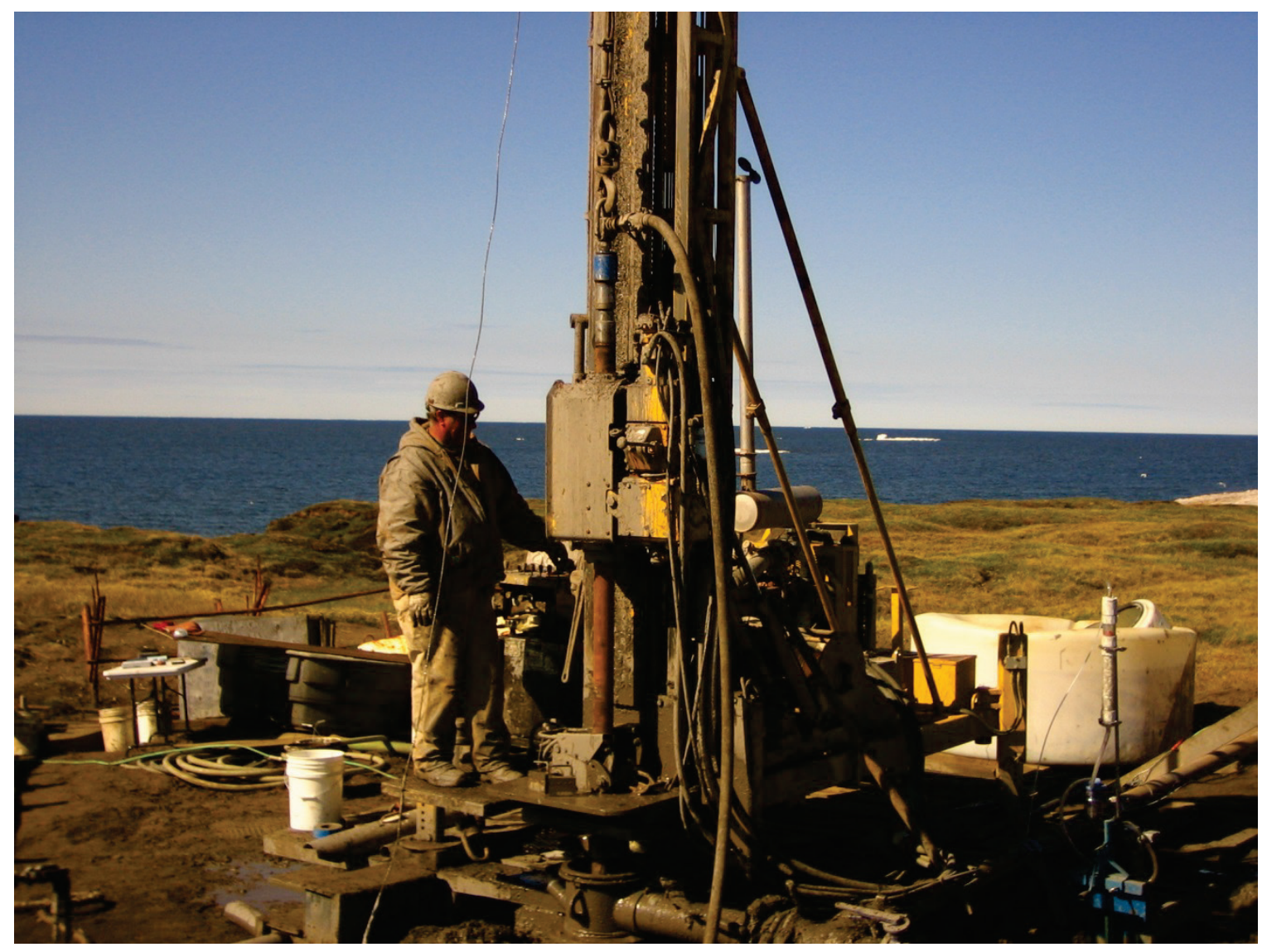

Figure 4. Alaska Rural Energy Project rig drilling the Wainwright-1 coalbed natural gas exploration core hole.

\section{Core Equipment, Handling, and Sample Collection}

Wainwright coring operations were conducted using an HWL (2.5-in.-diameter core) wireline system. Rather than removing drill rods from the well to retrieve core, the wireline system allowed for drilled core to be retrieved from the well-bore by lowering an "overshot" device attached to a thin steel cable through the core rods to the bottom of the hole. The overshot latched to the core barrel innertube containing the drilled core sample and was winched to land surface and laid out for core extraction. A second innertube was then lowered through the core rods and latched into place so that drilling could continue while the retrieved core was extracted, described, and sampled. After unthreading the "head" and "shoe" from either end of the retrieved innertube, the core was carefully extruded from the tube into a core tray using a 10-ft rod or, when the core was tight, a pressure-controlled fluid pump. Once in the tray, the core was washed, measured, described, sampled, and boxed (fig. 5). When coal was present in the core, it was immediately washed, measured, cut into 1-ft lengths, and placed in gas-desorption canisters. After weighing and sealing the canisters, they were placed in a thermal bath for desorption. Only after the gas-desorption process had started were coal-sample depth intervals determined and other critical data such as sample depth and weight, time off bottom, time at surface, and time canister sealed, entered into desorption spreadsheets. The remaining portion of the core was then measured, described, and boxed. 


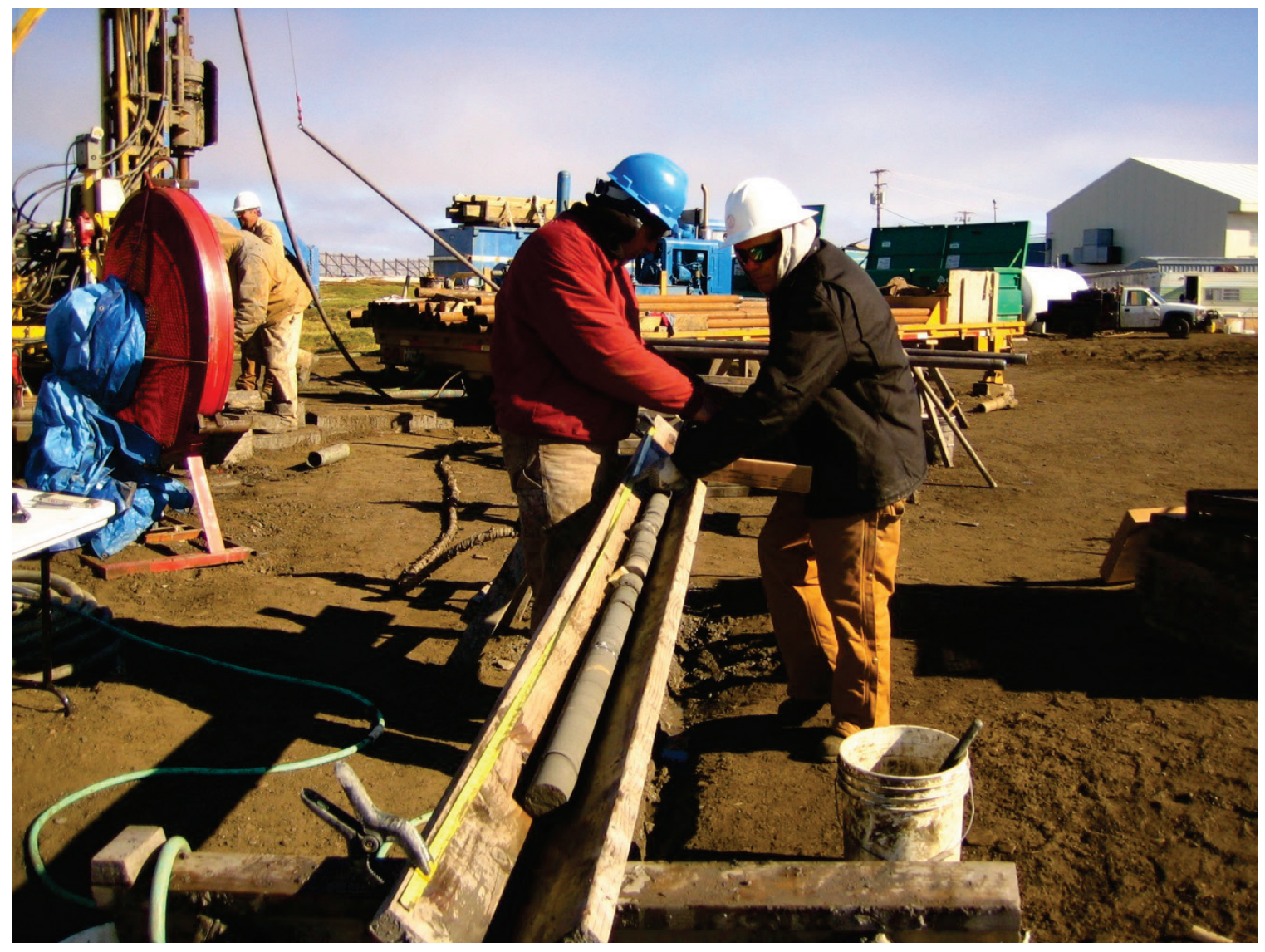

Figure 5. Project geologists describing and boxing core samples from the Wainwright-1 core hole.

A net thickness of $70 \mathrm{ft}$ of coal was penetrated in the Wainwright-1 well, with bed thickness ranging from several inches to $7.6 \mathrm{ft}$. Although all coal cores were not desorbed, most coalbeds with a thickness exceeding $1 \mathrm{ft}$ had at least one desorption sample taken. As drilling extended below the estimated depth of permafrost, two desorption samples were taken from several of the thicker coalbeds. Four 1-ft desorption samples were taken from the 7.6-ft-thick Wainwright coalbed penetrated at 1,242 ft. A total of twenty-five 1-ft coal samples were desorbed, ranging in depth from 105.0 to $1,477.5 \mathrm{ft}$. Core was drilled in full 10-ft runs whenever possible, and total core recovery was approximately 95 percent. A generalized lithologic core column was constructed by Stephen B. Roberts of the USGS and is included in cross sections depicted in Clark and others (2010). Detailed core descriptions and depositional interpretations were made by David L. LePain of the Alaska DGGS and Paul L. Decker of the Alaska Department of Natural Resources and are awaiting publication.

\section{Geophysical Logging}

Geophysical logs were collected using a project-owned Century Geophysical Corporation portable logging system. Due to the broken core rods lost in well Wainwright-1, natural gamma and density were the only logs that could be collected from the bottom of the well $(1,613 \mathrm{ft})$ to the top of the broken rods $(957 \mathrm{ft})$. The suite of 
natural gamma, density, resistivity, and caliper logs were collected from $957 \mathrm{ft}$ to land surface. Natural gamma, resistivity, and caliper logs were also collected from well Wainwright-2 from the bottom depth of 1,295 $\mathrm{ft}$ to land surface. A combination of the geophysical logs from wells Wainwright-1 and Wainwright-2 and a logged-well-location map are shown in plate 2 .

\section{Coal Desorption}

Coal-gas content was determined using direct desorption methods as described in Diamond and Levine (1981), Mavor and Nelson (1997), Diamond and Schatzel (1998), and the American Society for Testing and Materials (ASTM) Standard Practice for Determination of Gas Content of Coal (ASTM, 2011), among others. Cores were desorbed in sealed 3-in.-diameter polyvinyl chloride (PVC) canisters filled with distilled water to allow for zero headspace measurements as described in Barker and Dallegge (2005). Once canisters were sealed and placed in thermal baths (fig. 6), regular readings were taken for (1) time, (2) ambient temperature, (3) thermal bath temperature, (4) barometric pressure, and (5) desorbed-gas volume. In general, these readings were taken every 5 minutes for the first half-hour, every 10 minutes for the next hour, and every 15 minutes for the next 2 hours. Subsequent readings were taken at progressively longer intervals depending on the amount of gas being measured. The coal cores were still desorbing at the mid-July conclusion of field operations, and samples needed to be airfreighted from Wainwright to the USGS laboratory in Denver, Colo., for continued desorption. Ten days prior to shipping, the thermal bath temperatures were significantly increased to desorb as much gas as possible from the coal before shipment. Final readings were taken in Wainwright on July 14 with no additional readings taken until the canisters arrived in Denver on July 20. In Denver, the canisters were kept at room temperature (approximately $70^{\circ} \mathrm{F}$ ) and measured on a weekly, biweekly, and monthly basis until desorption was completed. The total desorption period for the majority of coal samples, especially those collected from depths in excess of $500 \mathrm{ft}$, was approximately 7 months. Data were entered into spreadsheets as described in Barker and others (2002), and measured values were corrected to standard temperature and pressure (STP), desorption graphs were generated, and lost-gas values were determined. 


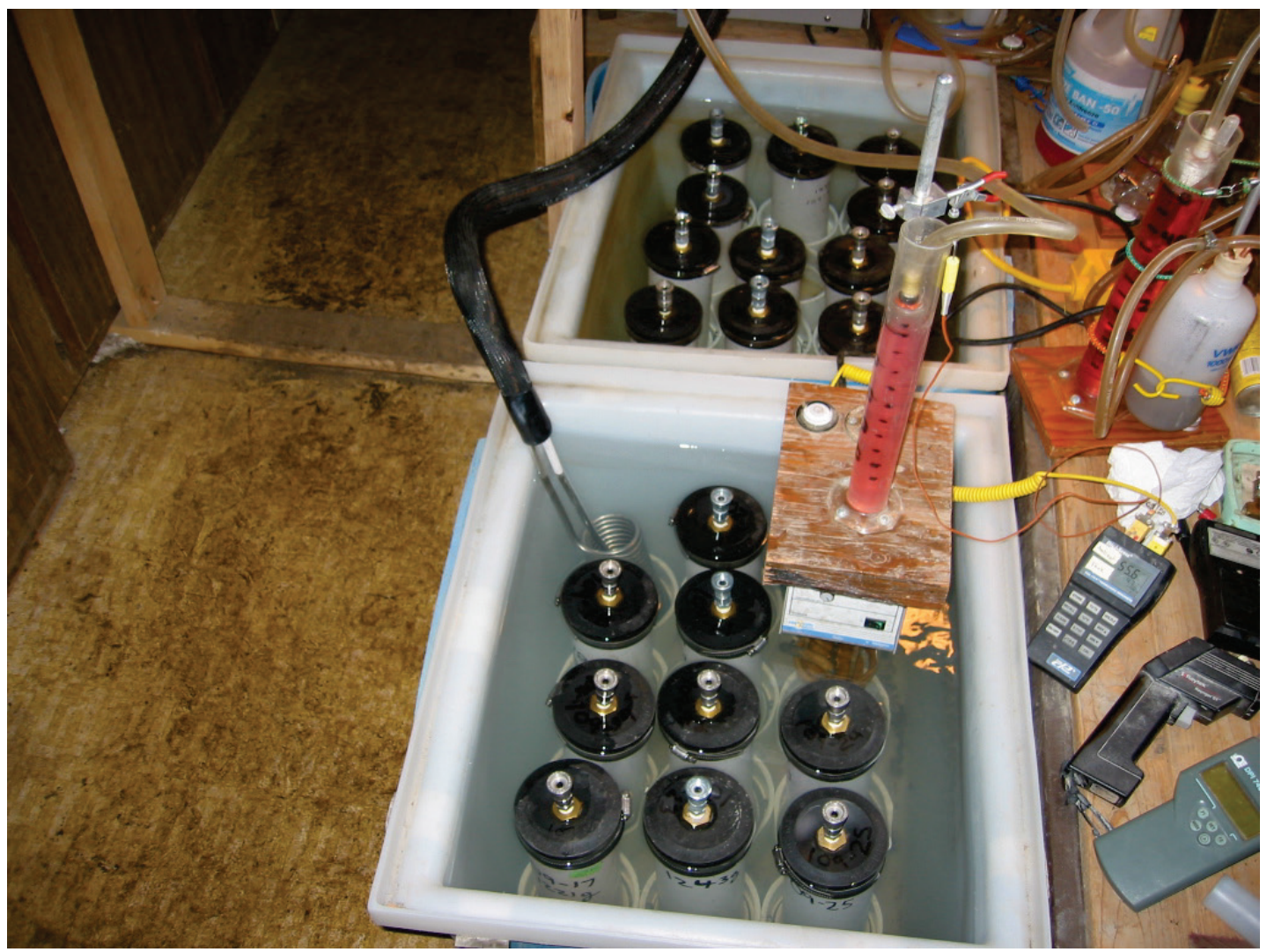

Figure 6. Wainwright-1 coal desorption canisters in thermal baths.

\section{Lost-Gas Estimate}

Desorption begins from a gas-bearing coal core as soon as the sample is lifted from well bottom (entered in the desorption spreadsheet as "time zero") and continues, unmeasured, at land surface until the sample is sealed in a desorption canister. The actual total sorbed-gas content of a coal sample is determined by adding this "lost-gas" value to the cumulative measured values. For this report, lost-gas values were calculated by examining the graph of cumulative desorbed gas versus the square root of elapsed time (the time between time zero and the desorption readings) and selecting the early-time points that plot in a linear manner. These points were used to establish a regression line that intersects the y-axis (time zero) at the negative lost-gas value (fig. 7). The absolute value of this number is added to the measured gas values to determine the actual desorbed-gas volume. By using a wireline system to quickly retrieve cores from the well, the lapsed time from when coal cores were lifted off the bottom to when they were sealed in desorption canisters was generally less than 30 minutes. 


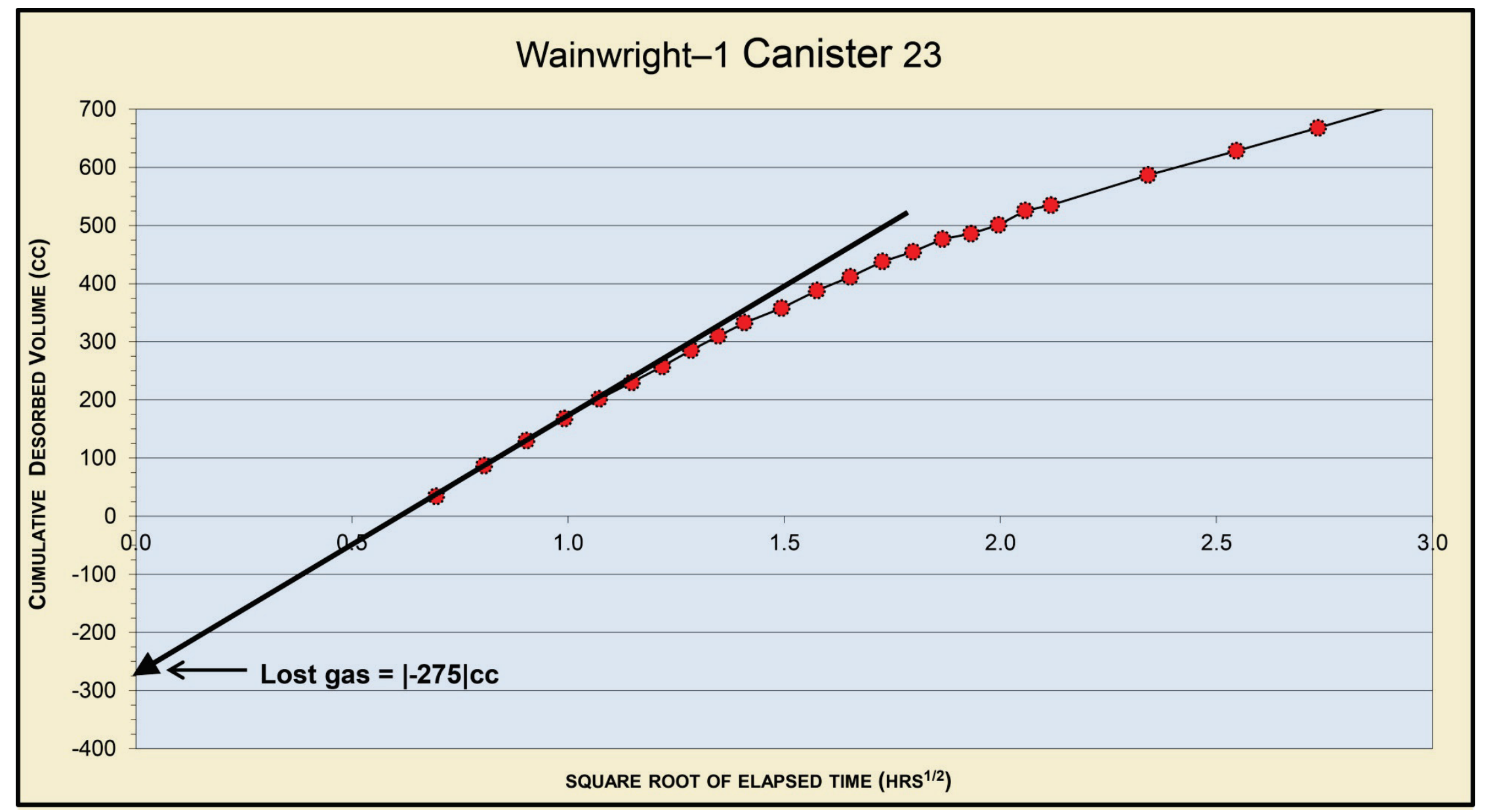

Figure 7. Graph depicting desorption regression line used to determine lost-gas value. Abbreviation: cc, cubic centimeters. 
Because gas-desorption rates are highly temperature-dependent, it is important that the early-time desorption readings used for lost-gas calculations be made at temperatures as close to the actual lost-gas desorption temperature as possible. Although a majority of coalbeds cored for this study occur within the zone of permafrost (for this report defined as ground temperature $\left.\leq 32^{\circ} \mathrm{F}\left(0{ }^{\circ} \mathrm{C}\right)\right)$, temperature measurements taken at the core face immediately after retrieval indicated that the core samples had thermally equilibrated to the drilling-fluid during the drilling and core-retrieval process. It was therefore assumed that lost-gas desorption occurred at drilling-fluid temperature rather than at coal reservoir temperature. Consequently, desorption baths were kept near drilling-fluid temperature, which generally ranged from $45-55^{\circ} \mathrm{F}\left(7.2-12.8^{\circ} \mathrm{C}\right)$. Although drilling-fluid temperatures did fluctuate over time (warmer ambient temperature equaled warmer drilling-fluid temperature), fluid temperatures generally remained in this range.

\section{Desorption Results}

With the exception of a 7.2-ft-thick coalbed penetrated just below $100 \mathrm{ft}$, all coalbeds penetrated in the Wainwright-1 well contained significant volumes of gas. Raw gas-desorption values ranged from a low of 52.8 standard cubic feet of gas per ton (scf/ton) of coal at a depth of $249 \mathrm{ft}$ to a high of $187.3 \mathrm{scf} /$ ton at a depth of 1,477 ft. On a dry-ash-free (DAF) basis, these raw numbers increased from 74.2 to $232.9 \mathrm{scf} /$ ton, respectively. Full gas-desorption values are given in table 1. 
Table 1. Summary of Wainwright-1 canister desorption results.

[Abbreviations: ft, foot; g, grams; cc, cubic centimeters; scf/ton, standard cubic feet per ton; DAF, dry-ashfree; STP, standard temperature and pressure]

\begin{tabular}{lccccrrrr}
\hline Canister & $\begin{array}{c}\text { Depth } \\
\text { interval } \\
\text { (ft) }\end{array}$ & $\begin{array}{c}\text { Coal mass } \\
\text { raw } \\
\text { (g) }\end{array}$ & $\begin{array}{c}\text { Coal mass } \\
\text { DAF } \\
\text { (g) }\end{array}$ & $\begin{array}{c}\text { Lost } \\
\text { gas } \\
\text { (cc) }\end{array}$ & $\begin{array}{r}\text { Desorbed } \\
\text { gas @ STP } \\
\text { (cc) }\end{array}$ & $\begin{array}{c}\text { Desorbed } \\
\text { gas } \\
\text { (scf/ton) }\end{array}$ & $\begin{array}{c}\text { DAF } \\
\text { gas } \\
\text { (scf/ton) }\end{array}$ \\
\hline W1-1 & from & \multicolumn{1}{c}{ to } & & & & & & \\
W1-2 & 105.0 & 106.0 & 1149 & 726 & 7 & 74 & 2.3 & 3.6 \\
W1-3 & 247.9 & 218.4 & 514 & 328 & 50 & 863 & 56.9 & 89.1 \\
W1-4 & 249.0 & 246.0 & 1095 & 757 & 200 & 2027 & 65.1 & 94.2 \\
W1-5 & 309.8 & 310.8 & 1089 & 732 & 120 & 1816 & 56.9 & 74.2 \\
W1-6 & 365.1 & 366.1 & 1132 & 683 & 120 & 1937 & 58.2 & 96.5 \\
W1-7 & 470.9 & 471.7 & 869 & 461 & 300 & 1526 & 67.2 & 126.9 \\
W1-8 & 478.2 & 479.3 & 1108 & 786 & 140 & 2697 & 81.9 & 115.5 \\
W1-9 & 512.4 & 513.3 & 1046 & 770 & 260 & 2808 & 93.9 & 127.5 \\
W1-10 & 569.8 & 570.8 & 1073 & 796 & 100 & 2784 & 86.0 & 116.0 \\
W1-11 & 700.7 & 701.7 & 1028 & 779 & 100 & 3343 & 107.2 & 141.4 \\
W1-12 & 787.6 & 788.6 & 1124 & 803 & 200 & 3903 & 116.8 & 163.5 \\
W1-13 & 899.1 & 900.0 & 1088 & 818 & 240 & 3852 & 120.4 & 160.0 \\
W1-14 & 906.0 & 906.9 & 1533 & 674 & 600 & 2496 & 64.6 & 147.0 \\
W1-15 & 957.7 & 958.7 & 1006 & 753 & 200 & 4455 & 148.1 & 197.8 \\
W1-16 & 956.1 & 957.1 & 976 & 742 & 250 & 3411 & 120.0 & 157.8 \\
W1-17 & 984.7 & 985.7 & 1701 & 1297 & 250 & 4489 & 89.1 & 116.9 \\
W1-18 & 983.0 & 984.0 & 1140 & 843 & 240 & 3959 & 117.9 & 159.3 \\
W1-19 & 1028.2 & 1029.2 & 1027 & 749 & 260 & 4277 & 141.4 & 193.9 \\
W1-20 & 1244.9 & 1245.9 & 1108 & 780 & 400 & 2283 & 77.5 & 110.1 \\
W1-21 & 1245.9 & 1246.9 & 1046 & 747 & 250 & 4911 & 157.9 & 221.0 \\
W1-22 & 1248.6 & 1249.6 & 891 & 559 & 600 & 3604 & 151.0 & 240.7 \\
W1-23 & 1250.9 & 1251.9 & 1079 & 875 & 275 & 4861 & 152.3 & 187.7 \\
W1-24 & 1313.6 & 1314.6 & 1006 & 715 & 200 & 4718 & 156.4 & 220.0 \\
W1-25 & 1477.5 & 1478.5 & 1073 & 863 & 200 & 6081 & 187.3 & 232.9 \\
\hline & & & & & & & &
\end{tabular}

\section{Desorbed-Gas and Mud-Gas Samples}

Desorbed-gas samples were collected from six desorption canisters with depths ranging from 245 to $1,477 \mathrm{ft}$. The samples were collected by fitting a $75-\mathrm{mm}$ stainless steel canister, at atmospheric pressure, inline between the PVC desorption canister and the manometer used to measure desorbed-gas volume. When a desorption measurement was made, gas released from the desorption canister flowed through the sample canister before entering the manometer. Closing the needle valve on each end of the sample canister thus captured a 75-mm volume of the desorbed gas. The gas samples were sent to Isotech Laboratories in Champaign, Ill., for compositional and isotopic analyses. Because the sample canisters were not purged of atmospheric gas prior to sampling, it is assumed that all oxygen $\left(\mathrm{O}_{2}\right)$ gas reported in the analyses was the result of atmospheric contamination (a gas sample collected during Wainwright coalbed production testing in 2009 contained only minor $\mathrm{O}_{2}(0.24$ percent $)$, thereby confirming this assumption). After 
normalizing data to an air-free basis, all samples had a methane $\left(\mathrm{CH}_{4}\right)$ content in excess of 99 mol-percent. As-received and normalized desorbed-gas compositional analyses are shown in table 2.

The $\delta^{13} \mathrm{C}_{1}$ of the six desorbed-gas samples ranges from -59.97 to $-53.97 \%$ oo with an average of $-56.38 \%$. The $\delta \mathrm{DC}_{1}$ for the samples ranges from -258.2 to $-244.1 \%$ and averages $-251.6 \%$. This isotopic signature suggests that the methane is a mixture of biogenically and thermogenically derived gases (Whiticar, 1999). The general increase in $\delta^{13} \mathrm{C}_{1}$ with depth may indicate an increased thermogenic component as depth increases. Desorbed-gas isotopic analyses are shown in table 3. 
Table 2. Wainwright-1 desorbed-gas compositional analysis.

[Abbreviations: \%, percent]

\begin{tabular}{|c|c|c|c|c|c|c|c|c|c|c|c|c|c|c|c|c|c|}
\hline \multicolumn{18}{|c|}{ Gas composition as-received } \\
\hline $\begin{array}{c}\text { Sample } \\
\text { name }\end{array}$ & $\begin{array}{c}\text { Sample } \\
\text { depth }\end{array}$ & $\begin{array}{c}\mathrm{He} \\
\% \\
\end{array}$ & $\begin{array}{l}\mathrm{H}_{2} \\
\% \\
\end{array}$ & $\begin{array}{l}\mathrm{Ar} \\
\% \\
\end{array}$ & $\begin{array}{l}\mathrm{O}_{2} \\
\%\end{array}$ & $\begin{array}{c}\mathrm{CO}_{2} \\
\%\end{array}$ & $\begin{array}{l}\mathrm{N}_{2} \\
\% \\
\end{array}$ & $\begin{array}{c}\text { CO } \\
\%\end{array}$ & $\begin{array}{l}\mathrm{C}_{1} \\
\% \\
\end{array}$ & $\begin{array}{l}\mathrm{C}_{2} \\
\%\end{array}$ & $\begin{array}{c}\mathrm{C}_{2} \mathrm{H}_{4} \\
\% \\
\end{array}$ & $\begin{array}{l}\mathrm{C}_{3} \\
\% \\
\%\end{array}$ & $\begin{array}{c}\mathrm{iC}_{4} \\
\% \\
\end{array}$ & $\begin{array}{c}\mathrm{nC}_{4} \\
\% \\
\end{array}$ & $\begin{array}{c}\mathrm{iC}_{5} \\
\% \\
\end{array}$ & $\begin{array}{c}\mathrm{nC}_{5} \\
\% \\
\end{array}$ & $\begin{array}{c}\mathrm{C}_{6}+ \\
\% \\
\end{array}$ \\
\hline W1-3 & 245.0 & 0.00 & 0.01 & 0.14 & 3.09 & 0.45 & 12.04 & 0.00 & 84.27 & 0.00 & 0.00 & 0.00 & 0.00 & 0.00 & 0.00 & 0.00 & 0.00 \\
\hline W1-6 & 365.1 & 0.00 & 0.02 & 0.05 & 0.96 & 0.32 & 3.92 & 0.00 & 94.72 & 0.01 & 0.00 & 0.00 & 0.00 & 0.00 & 0.00 & 0.00 & 0.00 \\
\hline W1-11 & 700.7 & 0.00 & 0.00 & 0.07 & 1.37 & 0.05 & 5.62 & 0.00 & 92.88 & 0.01 & 0.00 & 0.00 & 0.00 & 0.00 & 0.00 & 0.00 & 0.00 \\
\hline W1-19 & 1028.2 & 0.00 & 0.00 & 0.10 & 2.25 & 0.06 & 8.39 & 0.00 & 89.19 & 0.01 & 0.00 & 0.00 & 0.00 & 0.00 & 0.00 & 0.00 & 0.00 \\
\hline W1-22 & 1248.6 & 0.00 & 0.00 & 0.11 & 2.45 & 0.05 & 9.52 & 0.00 & 87.87 & 0.01 & 0.00 & 0.00 & 0.00 & 0.00 & 0.00 & 0.00 & 0.00 \\
\hline W1-25 & 1477.5 & 0.00 & 0.00 & 0.16 & 3.62 & 0.11 & 13.92 & 0.00 & 82.18 & 0.01 & 0.00 & 0.00 & 0.00 & 0.00 & 0.00 & 0.00 & 0.00 \\
\hline \multicolumn{18}{|c|}{ Gas composition with atmospheric contamination removed } \\
\hline $\begin{array}{c}\text { Sample } \\
\text { name }\end{array}$ & $\begin{array}{c}\text { Sample } \\
\text { depth }\end{array}$ & $\begin{array}{c}\mathrm{He} \\
\% \\
\end{array}$ & $\begin{array}{c}\mathrm{H}_{2} \\
\% \\
\end{array}$ & $\begin{array}{c}\mathrm{Ar} \\
\%\end{array}$ & $\begin{array}{l}\mathrm{O}_{2} \\
\%\end{array}$ & $\begin{array}{c}\mathrm{CO}_{2} \\
\%\end{array}$ & $\begin{array}{l}\mathrm{N}_{2} \\
\% \\
\end{array}$ & $\begin{array}{c}\text { CO } \\
\%\end{array}$ & $\begin{array}{l}\mathrm{C}_{1} \\
\%\end{array}$ & $\begin{array}{l}\mathrm{C}_{2} \\
\% \\
\end{array}$ & $\begin{array}{c}\mathrm{C}_{2} \mathrm{H}_{4} \\
\% \\
\end{array}$ & $\begin{array}{l}\mathrm{C}_{3} \\
\%\end{array}$ & $\begin{array}{c}\mathrm{iC}_{4} \\
\% \\
\end{array}$ & $\begin{array}{c}\mathrm{nC}_{4} \\
\%\end{array}$ & $\begin{array}{c}\mathrm{iC}_{5} \\
\% \\
\end{array}$ & $\begin{array}{c}\mathrm{nC}_{5} \\
\% \\
\end{array}$ & $\begin{array}{c}\mathrm{C}_{6}+ \\
\% \\
\end{array}$ \\
\hline W1-3 & 245.0 & 0.00 & 0.01 & 0.00 & 0.00 & 0.44 & 0.52 & 0.00 & 99.03 & 0.00 & 0.00 & 0.00 & 0.00 & 0.00 & 0.00 & 0.00 & 0.00 \\
\hline W1-6 & 365.1 & 0.00 & 0.02 & 0.01 & 0.00 & 0.32 & 0.36 & 0.00 & 99.30 & 0.01 & 0.00 & 0.00 & 0.00 & 0.00 & 0.00 & 0.00 & 0.00 \\
\hline W1-11 & 700.7 & 0.00 & 0.00 & 0.01 & 0.00 & 0.05 & 0.51 & 0.00 & 99.43 & 0.01 & 0.00 & 0.00 & 0.00 & 0.00 & 0.00 & 0.00 & 0.00 \\
\hline W1-19 & 1028.2 & 0.00 & 0.00 & 0.00 & 0.00 & 0.05 & 0.00 & 0.00 & 99.95 & 0.01 & 0.00 & 0.00 & 0.00 & 0.00 & 0.00 & 0.00 & 0.00 \\
\hline W1-22 & 1248.6 & 0.00 & 0.00 & 0.00 & 0.00 & 0.05 & 0.39 & 0.00 & 99.57 & 0.01 & 0.00 & 0.00 & 0.00 & 0.00 & 0.00 & 0.00 & 0.00 \\
\hline W1-25 & 1477.5 & 0.00 & 0.00 & 0.00 & 0.00 & 0.10 & 0.43 & 0.00 & 99.48 & 0.01 & 0.00 & 0.00 & 0.00 & 0.00 & 0.00 & 0.00 & 0.00 \\
\hline $\begin{array}{c}\text { Sample } \\
\text { name }\end{array}$ & $\begin{array}{c}\text { Sample } \\
\text { depth }\end{array}$ & $\begin{array}{c}\delta^{13} \mathrm{CO}_{2} \\
\% 0\end{array}$ & $\begin{array}{c}\delta^{13} C_{1} \\
\% 0\end{array}$ & $\begin{array}{c}\delta \mathrm{DC}_{1} \\
\% 0\end{array}$ & $\begin{array}{c}\text { Specific } \\
\text { gravity }\end{array}$ & BTU & & & & & & & & & & & \\
\hline W1-3 & 245.0 & -11.37 & -59.97 & -249.0 & 0.626 & 854 & & & & & & & & & & & \\
\hline W1-6 & 365.1 & -7.04 & -58.35 & -257.2 & 0.579 & 961 & & & & & & & & & & & \\
\hline W1-11 & 700.7 & & -56.99 & -258.2 & 0.586 & 942 & & & & & & & & & & & \\
\hline W1-19 & 1028.2 & & -53.97 & -244.1 & 0.603 & 904 & & & & & & & & & & & \\
\hline W1-22 & 1248.6 & & -54.10 & -251.7 & 0.608 & 891 & & & & & & & & & & & \\
\hline W1-25 & 1477.5 & -6.41 & -54.91 & -249.1 & 0.634 & 833 & & & & & & & & & & & \\
\hline
\end{tabular}


Table 3. Wainwright-1 as-received desorbed-gas isotopic analyses.

[Abbreviations: Btu, British thermal units; o/oo, per mil]

\begin{tabular}{lcccccc}
\hline $\begin{array}{c}\text { Sample } \\
\text { name }\end{array}$ & $\begin{array}{c}\text { Sample } \\
\text { depth }\end{array}$ & $\begin{array}{c}\boldsymbol{\delta}^{13} \mathrm{CO}_{2} \\
\%_{0}\end{array}$ & $\begin{array}{c}\boldsymbol{\delta}^{13} \mathrm{C}_{1} \\
{ }^{1}\end{array}$ & $\begin{array}{c}\delta \mathrm{\delta D}_{1} \\
\%_{0}\end{array}$ & $\begin{array}{c}\text { Specific } \\
\text { gravity }\end{array}$ & BTU \\
\hline $\mathrm{W} 1-3$ & 245.0 & -11.37 & -59.97 & -249.0 & 0.626 & 854 \\
$\mathrm{~W} 1-6$ & 365.1 & -7.04 & -58.35 & -257.2 & 0.579 & 961 \\
$\mathrm{~W} 1-11$ & 700.7 & & -56.99 & -258.2 & 0.586 & 942 \\
$\mathrm{~W} 1-19$ & 1028.2 & & -53.97 & -244.1 & 0.603 & 904 \\
$\mathrm{~W} 1-22$ & 1248.6 & & -54.10 & -251.7 & 0.608 & 891 \\
$\mathrm{~W} 1-25$ & 1477.5 & -6.41 & -54.91 & -249.1 & 0.634 & 833 \\
\hline
\end{tabular}

Mud-gas was monitored using a system rented from Mudlogging Systems, Inc., of Grand Junction, Colo. Mud-gas samples were collected at 100-ft intervals as well as from several gas-bearing coalbeds and sent to Isotech Laboratories for compositional and isotopic analyses. Mud-gas analyses are shown in table 4. 
Table 4. Wainwright-1 mud-gas analysis.

[Abbreviations: ppm, parts per million; $n-a$, not available]

\begin{tabular}{|c|c|c|c|c|c|c|c|c|c|c|c|c|c|c|c|c|c|c|c|}
\hline $\begin{array}{c}\text { Sample } \\
\text { name }\end{array}$ & $\begin{array}{l}\text { Sample } \\
\text { date }\end{array}$ & $\begin{array}{c}\text { Sample } \\
\text { time }\end{array}$ & $\begin{array}{l}\text { Depth } \\
\text { feet }\end{array}$ & $\begin{array}{c}\text { Gas } \\
\text { units }\end{array}$ & $\begin{array}{c}\mathrm{O}_{2}+\mathrm{Ar} \\
\mathrm{ppm}\end{array}$ & $\begin{array}{l}\mathrm{CO}_{2} \\
\mathrm{ppm}\end{array}$ & $\begin{array}{c}\mathrm{N}_{2} \\
\mathrm{ppm}\end{array}$ & $\begin{array}{c}\mathrm{CO} \\
\mathrm{ppm}\end{array}$ & $\begin{array}{c}\mathrm{C}_{1} \\
\mathrm{ppm}\end{array}$ & $\begin{array}{c}\mathrm{C}_{2} \\
\mathrm{ppm}\end{array}$ & $\begin{array}{l}\mathrm{C}_{2} \mathrm{H}_{4} \\
\mathrm{ppm}\end{array}$ & $\begin{array}{c}\mathrm{C}_{3} \\
\mathrm{ppm}\end{array}$ & $\begin{array}{l}\mathrm{C}_{3} \mathrm{H}_{6} \\
\mathrm{ppm}\end{array}$ & $\begin{array}{c}\mathrm{iC}_{4} \\
\mathrm{ppm}\end{array}$ & $\begin{array}{l}\mathrm{nC}_{4} \\
\mathrm{ppm}\end{array}$ & $\begin{array}{c}\mathrm{iC}_{5} \\
\mathrm{ppm}\end{array}$ & $\begin{array}{l}\mathrm{nC}_{5} \\
\mathrm{ppm}\end{array}$ & $\begin{array}{l}\mathrm{C}_{6+}+ \\
\mathrm{ppm}\end{array}$ & $\begin{array}{c}\delta^{13} C_{1} \\
\text { per mil }\end{array}$ \\
\hline W1-MG-1 & $6 / 14 / 2007$ & $13: 57$ & 100 & 0 & 213400 & 150 & 786400 & 40 & 29 & 1 & 0 & 0 & 0 & 0 & 0 & 0 & 0 & 1 & $\mathrm{n}-\mathrm{a}$ \\
\hline W1-MG-2 & $6 / 14 / 2007$ & 23:00 & 200 & 35.9 & 217400 & 140 & 779200 & 70 & 3170 & 0 & 0 & 0 & 0 & 0 & 0 & 0 & 0 & 0 & -60.3 \\
\hline W1-MG-3 & $6 / 15 / 2007$ & $11: 30$ & 220 & 280 & 207300 & 150 & 773000 & 70 & 19500 & 0 & 0 & 0 & 0 & 0 & 0 & 0 & 0 & 0 & -59.1 \\
\hline W1-MG-4 & $6 / 15 / 2007$ & $14: 53$ & 250 & 1340 & 186300 & 160 & 754100 & 110 & 59300 & 0 & 0 & 0 & 0 & 0 & 0 & 0 & 0 & 0 & -59.7 \\
\hline W1-MG-5 & $6 / 15 / 2007$ & $22: 05$ & 320 & 195 & 200800 & 170 & 784100 & 90 & 14800 & 2 & 0 & 0 & 0 & 0 & 0 & 0 & 0 & 0 & -57.8 \\
\hline W1-MG-6 & $6 / 16 / 2007$ & $6: 52$ & 400 & 46 & 200100 & 230 & 795200 & 40 & 4450 & 0 & 0 & 0 & 0 & 0 & 0 & 0 & 0 & 0 & -57.5 \\
\hline W1-MG-7 & $6 / 16 / 2007$ & $21: 30$ & 500 & 85 & 191700 & 290 & 801600 & 60 & 6390 & 1 & 0 & 0 & 0 & 0 & 0 & 0 & 0 & 0 & -56.9 \\
\hline W1-MG-8 & $6 / 17 / 2007$ & $9: 35$ & 600 & 6.5 & 219700 & 390 & 779900 & 0 & 7 & 0 & 0 & 0 & 0 & 0 & 0 & 0 & 0 & 0 & $\mathrm{n}-\mathrm{a}$ \\
\hline W1-MG-9 & $6 / 17 / 2007$ & $23: 17$ & 703 & 210 & 195400 & 340 & 746200 & 0 & 58100 & 0 & 0 & 0 & 0 & 0 & 0 & 0 & 0 & 0 & -54.7 \\
\hline W1-MG-10 & $6 / 18 / 2007$ & $12: 35$ & 805 & 80 & 204800 & 330 & 787600 & 130 & 7140 & 1 & 0 & 0 & 0 & 0 & 0 & 0 & 0 & 0 & -55.2 \\
\hline W1-MG-11 & $6 / 19 / 2007$ & $0: 31$ & 900 & 998 & 194200 & 390 & 759400 & 30 & 46000 & 5 & 0 & 1 & 0 & 0 & 0 & 0 & 0 & 0 & -54.3 \\
\hline W1-MG-12 & 6/19/2007 & $7: 45$ & 985 & 3350 & 167900 & 390 & 699300 & 0 & 132400 & 0 & 0 & 0 & 0 & 0 & 0 & 0 & 0 & 0 & -54.1 \\
\hline W1-MG-13 & $6 / 19 / 2007$ & $21: 40$ & 1000 & 165 & 190500 & 350 & 795500 & 60 & 13600 & 1 & 0 & 0 & 0 & 0 & 0 & 0 & 0 & 0 & -54.4 \\
\hline W1-MG-14 & $6 / 20 / 2007$ & $10: 57$ & 1100 & 247 & 195700 & 340 & 784600 & 0 & 19400 & 2 & 0 & 0 & 0 & 0 & 0 & 0 & 0 & 0 & -53.8 \\
\hline W1-MG-15 & $6 / 21 / 2007$ & $11: 55$ & 1200 & 65 & 203900 & 350 & 788200 & 0 & 7570 & 1 & 0 & 0 & 0 & 0 & 0 & 0 & 0 & 0 & -53.8 \\
\hline W1-MG-16 & $6 / 21 / 2007$ & $20: 10$ & 1250 & 1650 & 179500 & 490 & 722300 & 0 & 97700 & 0 & 0 & 0 & 0 & 0 & 0 & 0 & 0 & 0 & -53.2 \\
\hline W1-MG-17 & $6 / 22 / 2007$ & $5: 50$ & 1300 & 70 & 208200 & 400 & 787100 & 0 & 4250 & 1 & 0 & 0 & 0 & 0 & 0 & 0 & 0 & 0 & -54.3 \\
\hline W1-MG-18 & $6 / 24 / 2007$ & $15: 25$ & 1400 & 50 & 203300 & 230 & 791700 & 0 & 4770 & 0 & 0 & 0 & 0 & 0 & 0 & 0 & 0 & 0 & -53.6 \\
\hline W1-MG-19 & $6 / 26 / 2007$ & $6: 55$ & 1500 & 41 & 219200 & 330 & 780300 & 0 & 184 & 0 & 0 & 0 & 0 & 0 & 0 & 0 & 0 & 0 & $\mathrm{n}-\mathrm{a}$ \\
\hline W1-MG-20 & $6 / 27 / 2007$ & $13: 59$ & 1600 & 57 & 196600 & 440 & 797500 & 0 & 5460 & 1 & 0 & 0 & 0 & 0 & 0 & 0 & 0 & 0 & -52.2 \\
\hline
\end{tabular}




\section{Coal Analyses}

Once gas desorption was completed, splits from each of the 25 desorbed-coal cores were sent to Geochemical Testing in Somerset, Penn., for coal analyses. Analyses run included proximate (moisture, ash, volatile matter, fixed carbon), ultimate (carbon, hydrogen, oxygen, nitrogen, sulfur), Btu, free-swelling index, equilibrium moisture, specific gravity, and forms of sulfur. Moist, mineral-matter-free Btu and coal rank were determined according to ASTM standard specifications for classification of coals by rank (ASTM, 1981). On this basis, coal rank ranged from subbituminous $C$ to subbituminous A. Coal analyses are shown in table 5 . 
Table 5. As-received geochemical analysis of well Wainwright-1 desorbed-coal samples.

[Abbreviations: ft, feet; Wt.-\%, weight percent; lb, pound; Btu, British thermal units; Sub A, subbituminous A; Sub B, subbituminous B; Sub C, subbituminous C]

\begin{tabular}{|c|c|c|c|c|c|c|c|c|c|c|c|c|c|}
\hline \multicolumn{14}{|c|}{ Wainwright-1 } \\
\hline Sample name & W1-1 & W1-2 & W1-3 & W1-4 & W1-5 & W1-6 & W1-7 & W1-8 & W1-9 & W1-10 & W1-11 & W1-12 & W1-13 \\
\hline Sample depth (ft) & 105 & 218 & 245 & 249 & 310 & 365 & 471 & 478 & 512 & 570 & 701 & 788 & 899 \\
\hline Air Dry Loss: Wt.-\% & 22.21 & 20.64 & 21.99 & 22.75 & 23.87 & 20.6 & 19.77 & 21.58 & 21.27 & 19.16 & 19.46 & 21.27 & 18.01 \\
\hline Residual Moisture: Wt.-\% & 4.18 & 4.39 & 3.76 & 5.19 & 3.52 & 4.21 & 3.51 & 4.95 & 3.91 & 4.57 & 3.42 & 3.95 & 3.65 \\
\hline Moisture: Wt.-\% & 25.46 & 24.12 & 24.92 & 26.76 & 26.55 & 23.94 & 22.59 & 25.46 & 24.35 & 22.85 & 22.21 & 24.38 & 21 \\
\hline Ash: Wt.-\% & 11.35 & 12.05 & 5.98 & 2.15 & 6.27 & 15.76 & 24.41 & 3.6 & 2.02 & 2.98 & 1.97 & 4.16 & 3.8 \\
\hline Volatile Matter: Wt.-\% & 26.35 & 26.83 & 26.05 & 27.28 & 26.77 & 24.33 & 21.2 & 28.35 & 28.42 & 31.11 & 30.51 & 29.89 & 29.65 \\
\hline Fixed Carbon: Wt.\% & 36.84 & 37 & 43.05 & 43.81 & 40.41 & 35.97 & 31.8 & 42.59 & 45.21 & 43.06 & 45.31 & 41.57 & 45.55 \\
\hline Hydrogen: Wt.\% & 3.21 & 3.39 & 3.26 & 3.54 & 3.36 & 3.07 & 2.62 & 3.6 & 3.64 & 3.89 & 3.94 & 3.74 & 3.79 \\
\hline Carbon: Wt.-\% & 46.4 & 47.46 & 52.76 & 53.39 & 50.56 & 45.62 & 39.99 & 54.02 & 56.64 & 56.71 & 58.19 & 54.8 & 58.15 \\
\hline Nitrogen: Wt.-\% & 1.1 & 1.11 & 0.91 & 1.38 & 1.05 & 1.05 & 0.69 & 1.06 & 0.99 & 1.22 & 1.3 & 1.14 & 1.16 \\
\hline Sulfur: Wt.-\% & 0.35 & 0.63 & 0.38 & 0.49 & 0.4 & 0.45 & 0.38 & 0.48 & 0.42 & 0.51 & 0.54 & 0.57 & 0.84 \\
\hline Oxygen: Wt.-\% & 12.13 & 11.24 & 11.79 & 12.29 & 11.81 & 10.11 & 9.32 & 11.78 & 11.94 & 11.84 & 11.85 & 11.21 & 11.26 \\
\hline Heating Value: Btu/lb & 7905 & 8209 & 8973 & 8930 & 8727 & 7756 & 6845 & 9364 & 9767 & 9906 & 10188 & 9568 & 10160 \\
\hline Sulfate Sulfur: Wt.-\% & 0.02 & 0.02 & 0.02 & 0.02 & 0.03 & 0.02 & 0.02 & 0.01 & 0.03 & 0.01 & 0.03 & 0.04 & 0 \\
\hline Pyritic Sulfur: Wt.-\% & 0.03 & 0.01 & 0.02 & 0.02 & 0 & 0.01 & 0.01 & 0 & 0 & 0.02 & 0.01 & 0.01 & 0.1 \\
\hline Organic Sulfur: Wt.-\% & 0.3 & 0.6 & 0.34 & 0.45 & 0.37 & 0.42 & 0.35 & 0.47 & 0.39 & 0.48 & 0.5 & 0.52 & 0.74 \\
\hline Equilibrium Moisture: Wt.-\% & 24.83 & 22.39 & 21.28 & 24.29 & 23.72 & 22.21 & 17.14 & 22.85 & 20.83 & 20.86 & 20.55 & 20.86 & 18.37 \\
\hline Lbs Sulfur/Million BTU & 0.44 & 0.77 & 0.42 & 0.55 & 0.46 & 0.58 & 0.56 & 0.51 & 0.43 & 0.51 & 0.53 & 0.6 & 0.83 \\
\hline True Specific Gravity & 1.56 & 1.55 & 1.5 & 1.48 & 1.5 & 1.63 & 1.73 & 1.46 & 1.46 & 1.46 & 1.49 & 1.48 & 1.43 \\
\hline MoistMineralMatterFree: BTU & 9009 & 9439 & 9594 & 9142 & 9362 & 9348 & 9296 & 9745 & 9987 & 10239 & 10413 & 10021 & 10602 \\
\hline Apparent Rank: & Sub C & Sub C & Sub B & Sub C & Sub C & Sub C & Sub C & Sub B & Sub B & Sub B & Sub B & Sub B & Sub A \\
\hline
\end{tabular}


Table 5. As-received geochemical analysis of well Wainwright-1 desorbed-coal samples.-Continued

[Abbreviations: ft, feet; Wt.-\%, weight percent; lb, pound; Btu, British thermal units; Sub A, subbituminous A; Sub B, subbituminous B; Sub C, subbituminous C]

\begin{tabular}{|c|c|c|c|c|c|c|c|c|c|c|c|c|}
\hline \multicolumn{13}{|c|}{ Wainwright-1 } \\
\hline Sample name & W1-14 & W1-15 & W1-16 & W1-17 & W1-18 & W1-19 & W1-20 & W1-21 & W1-22 & W1-23 & W1-24 & W1-25 \\
\hline Sample depth (ft) & 906 & 958 & 957 & 985 & 983 & 1028 & 1245 & 1246 & 1249 & 1251 & 1314 & 1478 \\
\hline Air Dry Loss: Wt.- $\%$ & 19.93 & 18.09 & 18.71 & 19.42 & 21.07 & 17.26 & 17.81 & 24.11 & 18.88 & 14.28 & 23.82 & 14.97 \\
\hline Residual Moisture: Wt.-\% & 3.57 & 3.28 & 4.07 & 3.21 & 4.14 & 3.69 & 3.93 & 3.29 & 3.91 & 3.42 & 4.01 & 3.11 \\
\hline Moisture: Wt.-\% & 22.79 & 20.78 & 22.02 & 22.01 & 24.34 & 20.31 & 21.04 & 26.61 & 22.05 & 17.21 & 26.87 & 17.61 \\
\hline Ash: Wt.-\% & 33.24 & 4.36 & 1.93 & 1.76 & 1.69 & 6.78 & 8.58 & 1.94 & 15.22 & 1.67 & 2.03 & 1.96 \\
\hline Fixed Carbon: Wt.\% & 24.1 & 41.84 & 44.4 & 43.57 & 45.15 & 41.08 & 39.5 & 42.16 & 34.94 & 46.1 & 43.01 & 44.26 \\
\hline Hydrogen: Wt.\% & 2.51 & 4.13 & 3.99 & 4.01 & 3.7 & 3.87 & 3.9 & 3.77 & 3.52 & 4.41 & 3.73 & 4.45 \\
\hline Carbon: Wt.-\% & 32.45 & 57.97 & 59.53 & 59.14 & 56.76 & 55.55 & 53.77 & 54.91 & 47.64 & 62.54 & 54.88 & 61.96 \\
\hline Nitrogen: Wt.-\% & 0.77 & 1.27 & 1.12 & 1.2 & 1.37 & 1.19 & 1.22 & 1.25 & 1.18 & 1.36 & 1.16 & 1.48 \\
\hline Sulfur: Wt.-\% & 0.35 & 0.46 & 0.42 & 0.37 & 0.39 & 0.43 & 0.55 & 0.39 & 0.32 & 0.34 & 0.53 & 1.34 \\
\hline Oxygen: Wt.-\% & 7.89 & 11.03 & 10.99 & 11.51 & 11.75 & 11.87 & 10.94 & 11.13 & 10.07 & 12.47 & 10.8 & 11.2 \\
\hline Sulfate Sulfur: Wt.-\% & 0.02 & 0 & 0.01 & 0 & 0.02 & 0 & 0 & 0.01 & 0.02 & 0.01 & 0.02 & 0.03 \\
\hline Pyritic Sulfur: Wt.-\% & 0.01 & 0 & 0 & 0.01 & 0.01 & 0.01 & 0.01 & 0.01 & 0.01 & 0.01 & 0.01 & 0.65 \\
\hline Organic Sulfur: Wt.-\% & 0.32 & 0.46 & 0.41 & 0.36 & 0.36 & 0.42 & 0.54 & 0.37 & 0.29 & 0.32 & 0.5 & 0.66 \\
\hline Equilibrium Moisture: Wt.-\% & 16.54 & 17.12 & 16.54 & 18.88 & 21.42 & 17.88 & 15.48 & 17.32 & 15.62 & 15.3 & 17.69 & 12.97 \\
\hline Lbs Sulfur/Million BTU & 0.61 & 0.45 & 0.4 & 0.36 & 0.4 & 0.44 & 0.57 & 0.41 & 0.38 & 0.31 & 0.55 & 1.22 \\
\hline True Specific Gravity & 1.79 & 1.42 & 1.38 & 1.43 & 1.46 & 1.5 & 1.44 & 1.41 & 1.58 & 1.48 & 1.47 & 1.45 \\
\hline MoistMineralMatterFree: BTU & 8943 & 10795 & 10670 & 10522 & 10031 & 10440 & 10588 & 9834 & 10160 & 11291 & 9902 & 11237 \\
\hline Apparent Rank: & Sub C & Sub A & Sub A & Sub A & Sub B & Sub B & Sub A & Sub B & Sub B & Sub A & Sub B & Sub A \\
\hline
\end{tabular}




\section{Isotherm Analyses}

Isotherm analyses are used to determine the maximum volume of gas that a coal body can adsorb at reservoir temperature and pressure. By introducing methane gas to a crushed, gas-free, coal sample maintained at reservoir temperature, and measuring the volume required to saturate the sample at increasing pressures, a sorption isotherm curve can be constructed. Using this curve in conjunction with desorbed-gas measurements allows a determination to be made as to whether a coalbed reservoir is saturated with gas and, if not, the pressure drop required for saturation to occur and desorption to begin.

Once canister desorption was complete, samples from 9 of the 25 desorbed-coal cores were sent to Ticora Laboratories in Golden, Colo., for isotherm analysis. Sample depths ranged from 249 to 1,477.5 ft. Estimated reservoir temperatures were based on the temperature log collected from well Wainwright-2 by UAF on Sept. 19, 2007, and ranged from a low of $19^{\circ} \mathrm{F}$ at $249 \mathrm{ft}$ to a high of $38^{\circ} \mathrm{F}$ at $1,477.5 \mathrm{ft}$. Reservoir pressures were calculated by taking the sample depth, subtracting the approximate local potentiometric surface of $30 \mathrm{ft}$ (Edwin Weeks, USGS, oral commun., Nov. 13, 2007), multiplying the result by the freshwater hydrostatic pressure of 0.433 pounds per square inch (psi), and adding a sea-level atmospheric pressure of 14.7 psi. The resulting reservoir pressures ranged from a low of $110 \mathrm{psi}$ at a depth of $249 \mathrm{ft}$ to a high of $641 \mathrm{psi}$ at a depth of $1,477.5 \mathrm{ft}$.

The isotherm graphs are shown in figures 8 through 18 . Theoretically, the Langmuir curve indicates the maximum volume of sorbed-gas a coal body can hold at reservoir temperature and a given pressure. Therefore, assuming that reservoir temperature and pressure are correct, a coal sample should not be capable of desorbing more gas than what the Langmuir curve indicates its maximum storage limit to be. However, in the nine samples on which isotherm analyses were conducted, the amount of gas desorbed from the core samples consistently exceeded their calculated storage capacity, with excesses ranging from a low of 9 percent to a high of 93 percent. Due to the discordant nature of the desorption and isotherm data, samples from two of the previously analyzed coal cores were sent to RMB Earth Science Consultants, Inc. (RMB), in Delta, British Columbia, for comparative isotherm analyses. The RMB reported storage values were 78 percent higher for a sample from a depth of 1,028 $\mathrm{ft}$ (130 vs. $73 \mathrm{scf} /$ ton) and 60 percent higher for a sample from a depth of 1,246 ft (230 versus $144 \mathrm{scf} /$ ton) than the Ticora reported values (figs. 13-16). The RMB results indicated that the measured desorbed-gas content of the coalbed at $1,028 \mathrm{ft}$ was 8 percent greater than its measured storage capacity (fig. 14), whereas the measured desorbed-gas content of the coalbed at 1,246 ft was 31 percent less than its measured storage capacity (fig 16).

It is unclear why such unusual and discordant results occurred. If, in fact, reservoir temperature was significantly overestimated and (or) reservoir pressure was significantly underestimated, the desorption results would better fit the Langmuir curves. However, the temperature log collected from well Wainwright-2 provided a detailed temperature profile to a depth of 1,260 ft, and hydraulic testing in the same well provided an accurate reservoir pressure for the 1,242-ft-deep Wainwright coalbed. It was postulated that perhaps some of the core samples contained some amount of methane-rich gas hydrate that, upon melting, would release appreciable amounts of additional methane 
gas in the desorption canisters. However, no sign of gas hydrate was ever noted during visual inspection and, more telling, desorption data did not indicate elevated early-time gas release as would be expected if hydrates were indeed present in the core.

Additionally, many of the coalbeds, including three from which isotherms were run, are located above the area's estimated hydrate-stability zone (Tim Collett, USGS, written commun., Oct. 12, 2007). Given the discrepancies, it was postulated that having isotherm analyses run at such anomalously low temperatures might lead to analytical complications and inaccuracies and that this was the likely explanation for the unusual results. Although it cannot be said with certainty, for this report, it is assumed that the coal reservoirs are either at or near gas-saturation levels. The production of methane in the early stages of subsequent-year reservoir testing (see 2008 and 2009 project results) suggests that the Wainwright coalbed is, in fact, saturated with methane gas. 


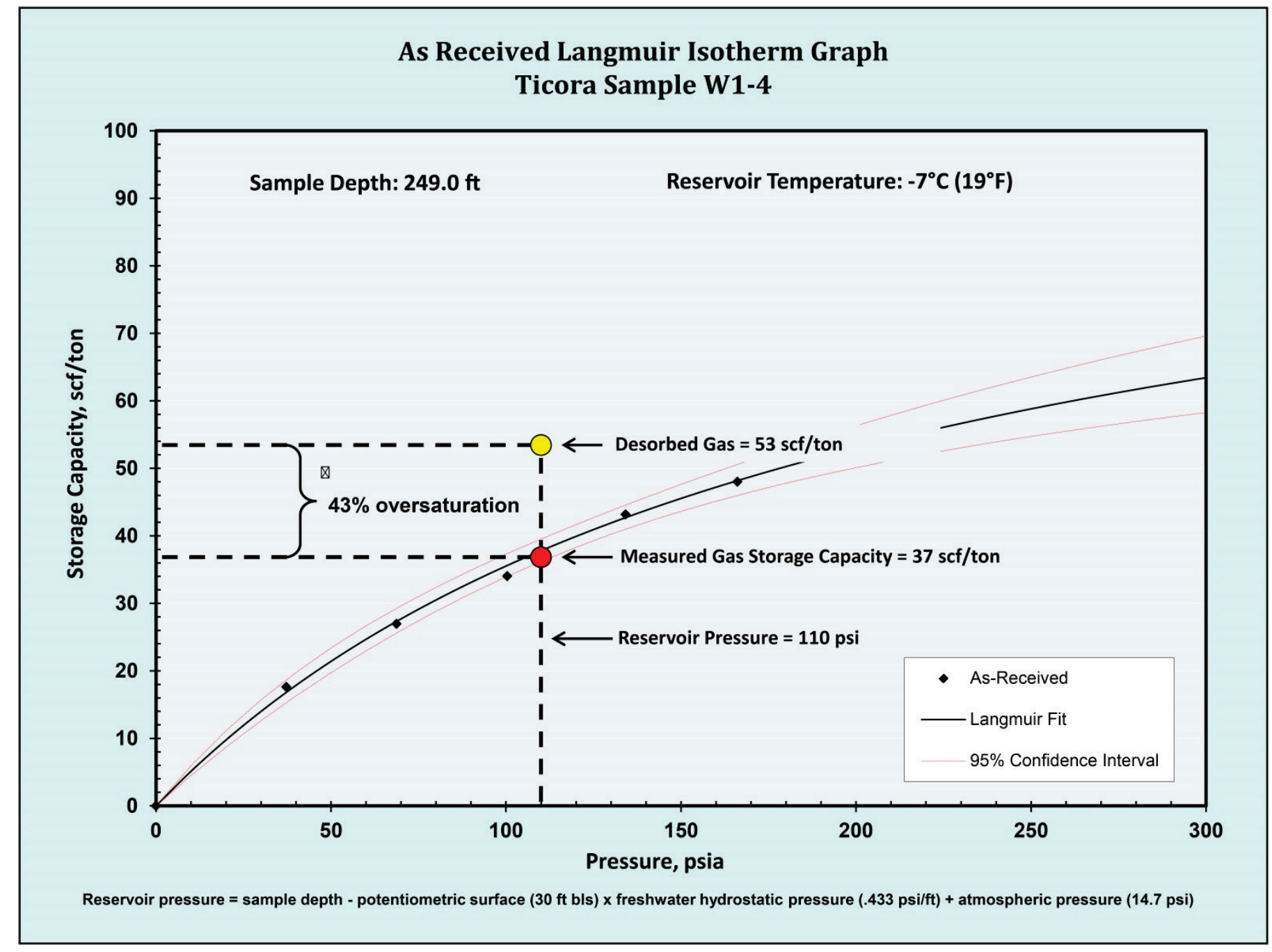

Figure 8. Isotherm chart from well Wainwright-1, desorption canister W1-4, analyzed by Ticora Laboratories. Abbreviations: scf/ton, standard cubic feet per ton; psi, pounds per square inch; psia, pounds per square inch absolute. 


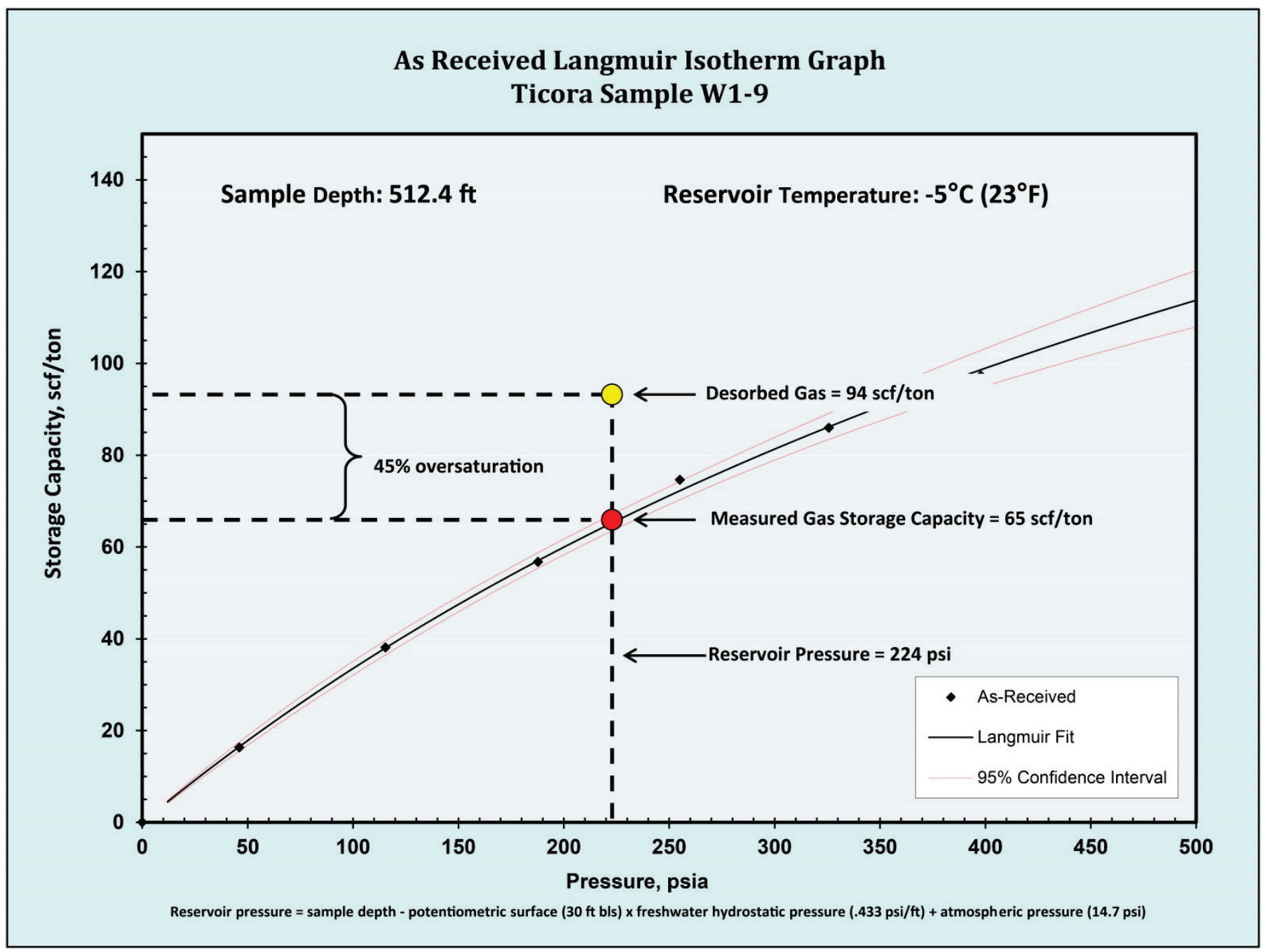

Figure 9. Isotherm chart from well Wainwright-1, desorption canister W1-9, analyzed by Ticora Laboratories. Abbreviations: scf/ton, standard cubic feet per ton; psi, pounds per square inch; psia, pounds per square inch absolute. 


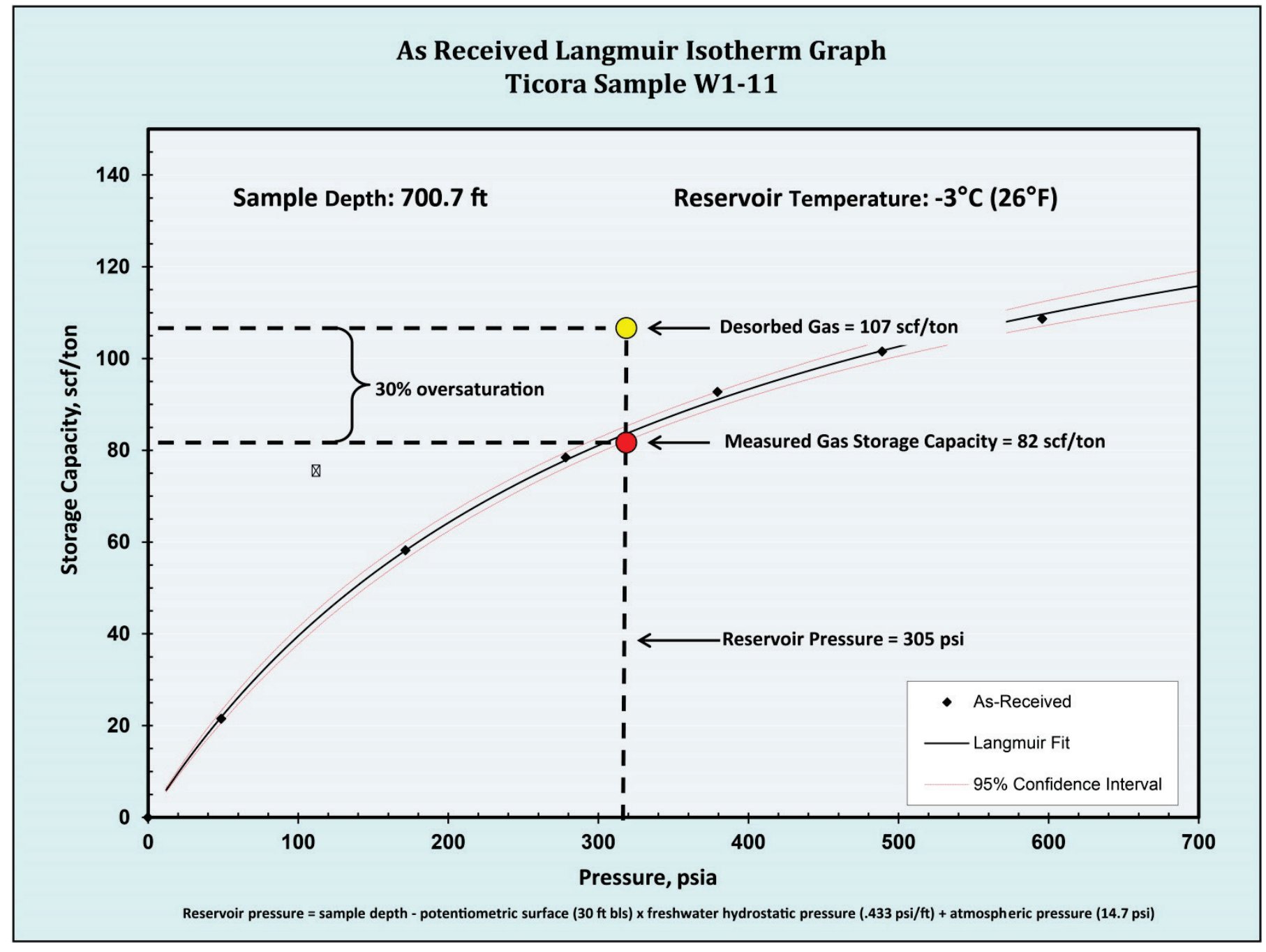

Figure 10. Isotherm chart from well Wainwright-1, desorption canister W1-11, analyzed by Ticora Laboratories. Abbreviations: scf/ton, standard cubic feet per ton; psi, pounds per square inch; psia, pounds per square inch absolute. 


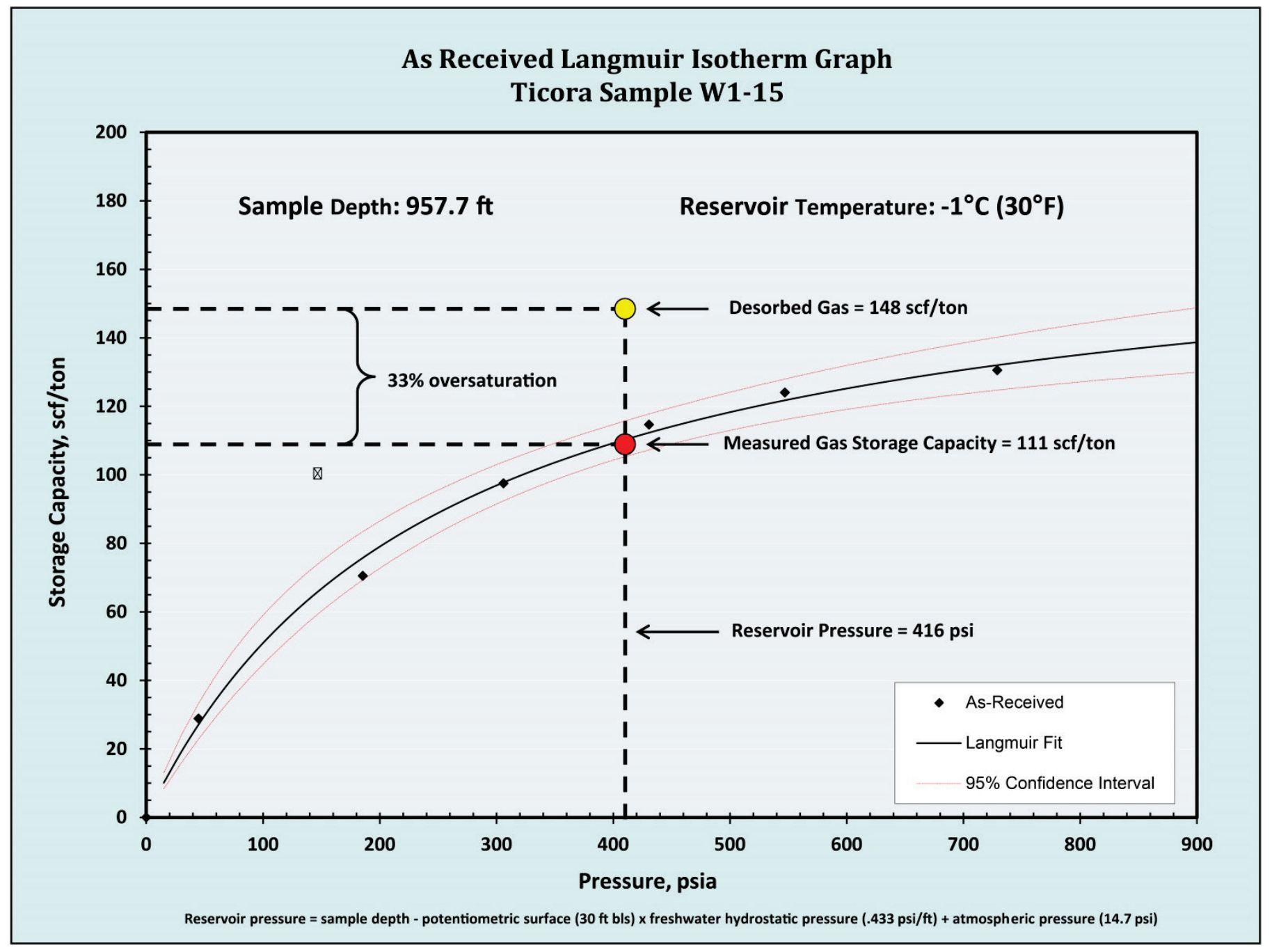

Figure 11. Isotherm chart from well Wainwright-1, desorption canister W1-15, analyzed by Ticora Laboratories. Abbreviations: scf/ton, standard cubic feet per ton; psi, pounds per square inch; psia, pounds per square inch absolute. 


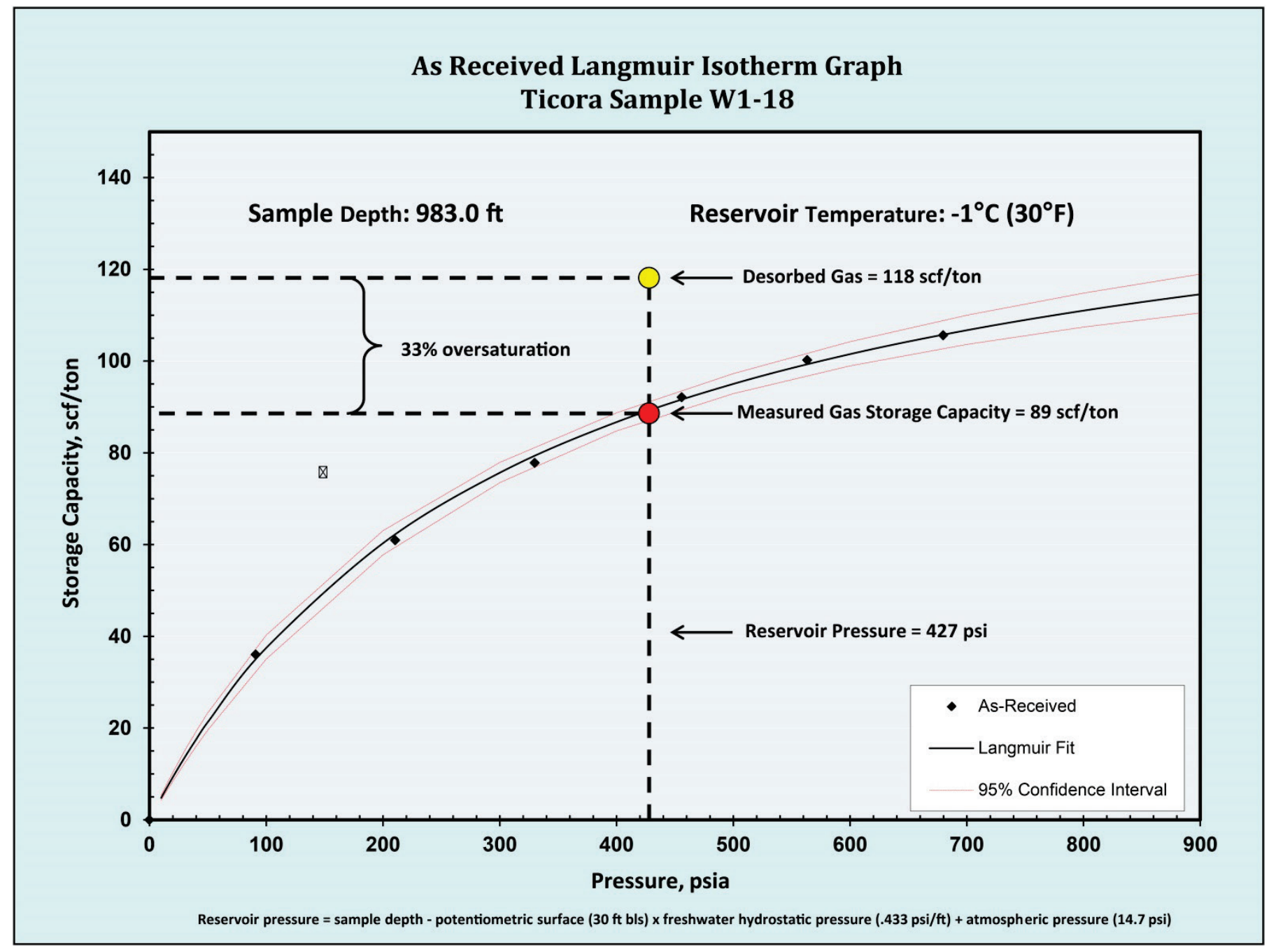

Figure 12. Isotherm chart from well Wainwright-1, desorption canister W1-18, analyzed by Ticora Laboratories. Abbreviations: scf/ton, standard cubic feet per ton; psi, pounds per square inch; psia, pounds per square inch absolute. 


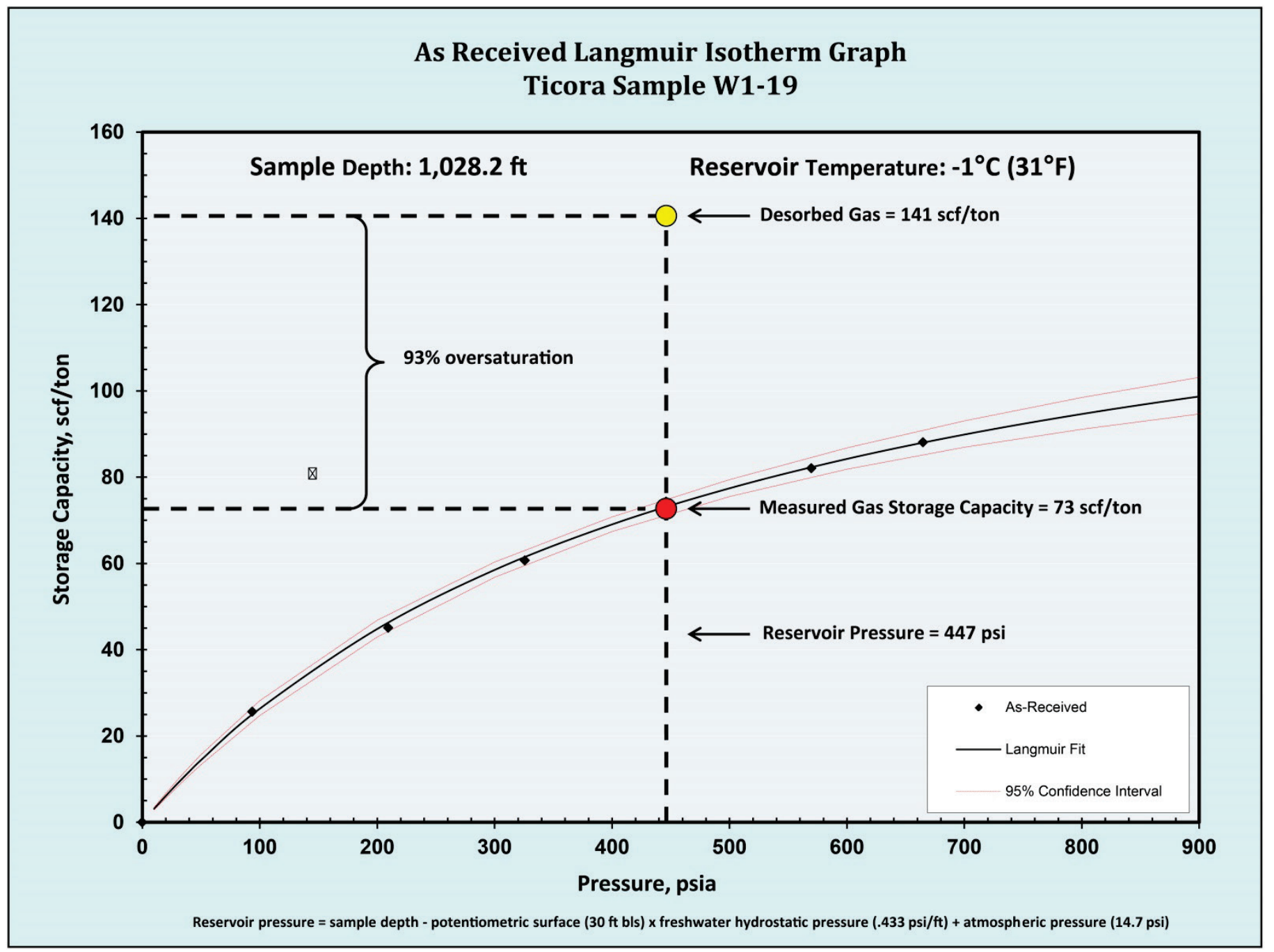

Figure 13. Isotherm chart from well Wainwright-1, desorption canister W1-19, analyzed by Ticora Laboratories. Abbreviations: scf/ton, standard cubic feet per ton; psi, pounds per square inch; psia, pounds per square inch absolute. 


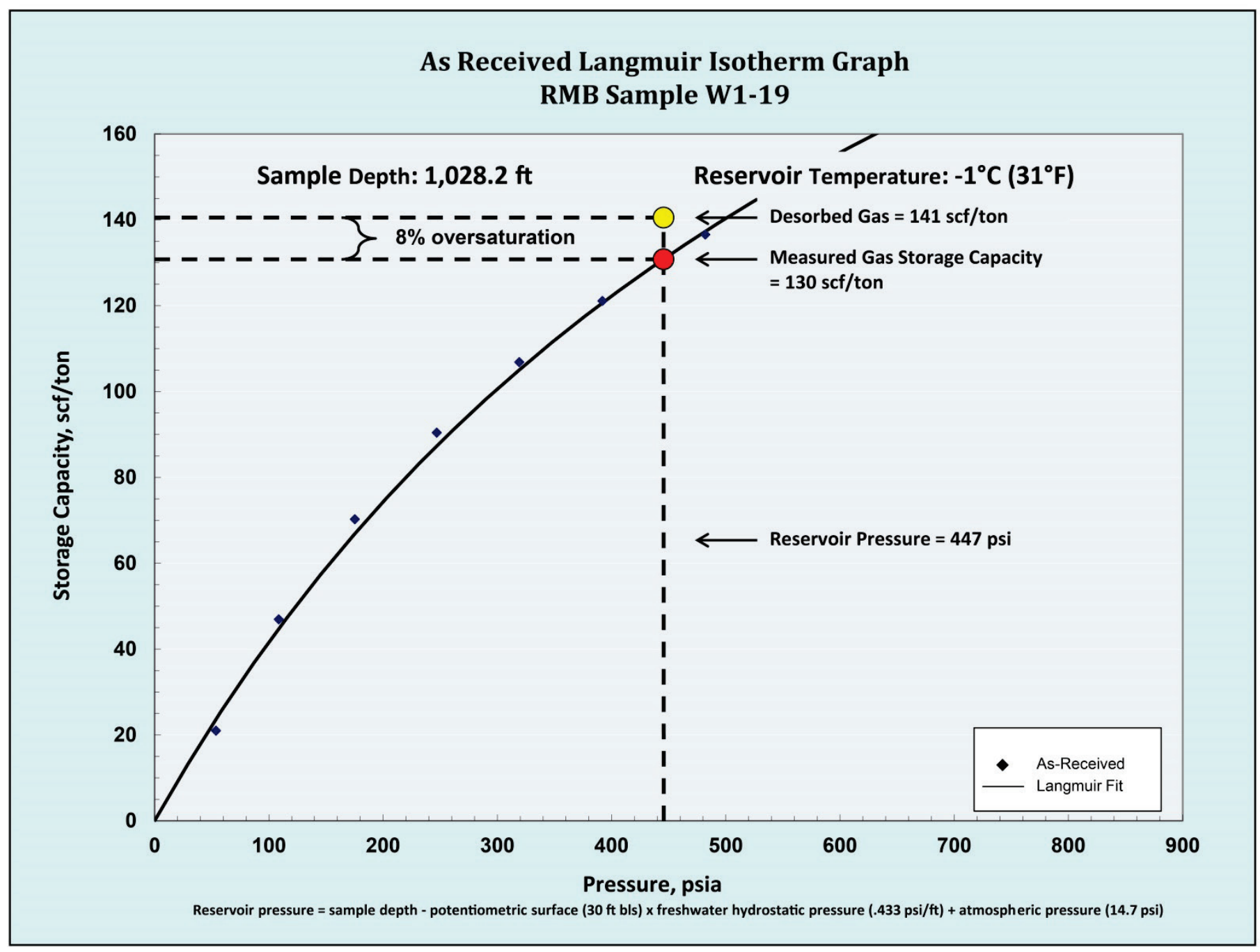

Figure 14. Isotherm chart from well Wainwright-1, desorption canister W1-19, analyzed by RMB Earth Science Consultants, Inc. Abbreviations: scf/ton, standard cubic feet per ton; psi, pounds per square inch; psia, pounds per square inch absolute. 


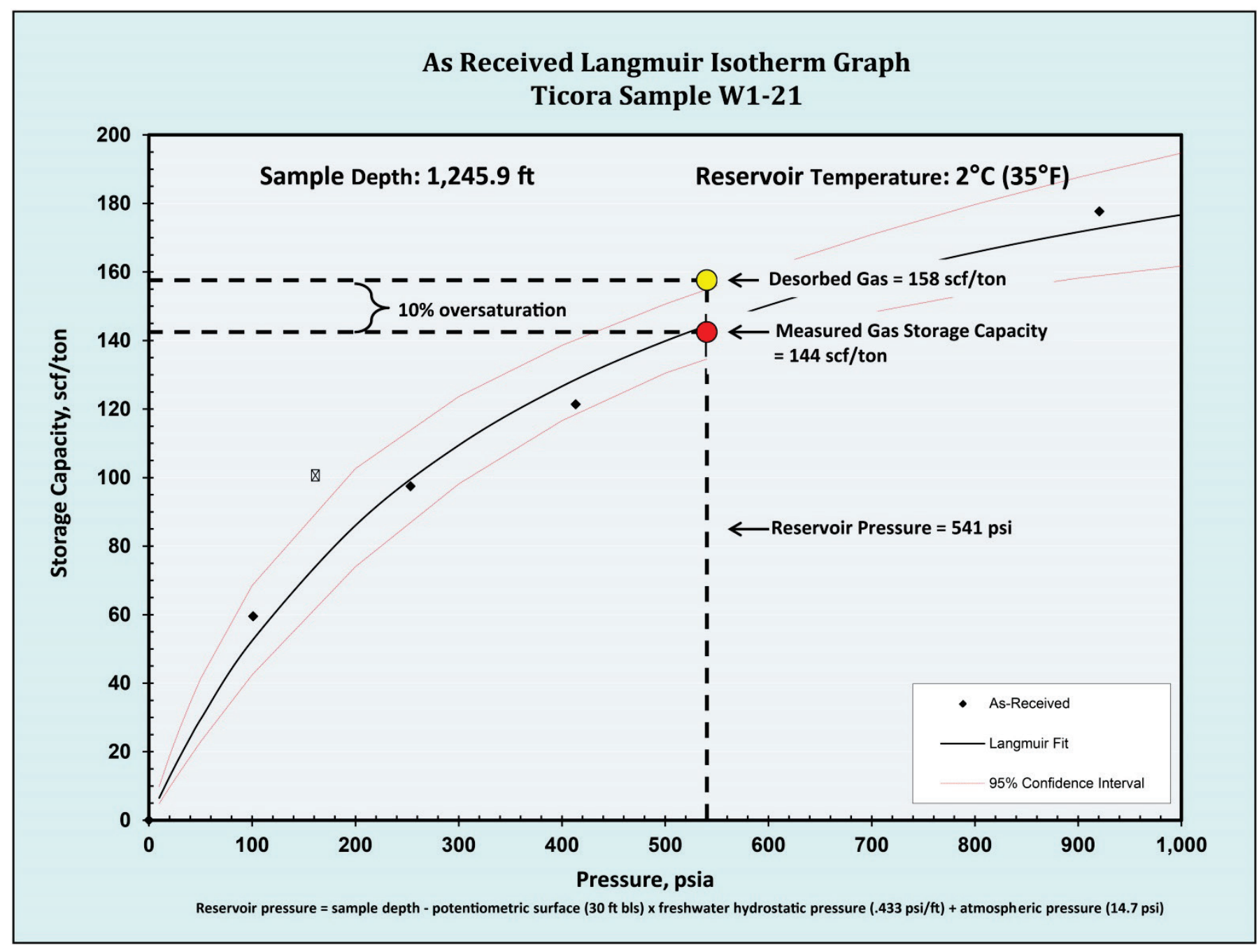

Figure 15. Isotherm chart from well Wainwright-1, desorption canister W1-21, analyzed by Ticora Laboratories. Abbreviations: scf/ton, standard cubic feet per ton; psi, pounds per square inch; psia, pounds per square inch absolute. 


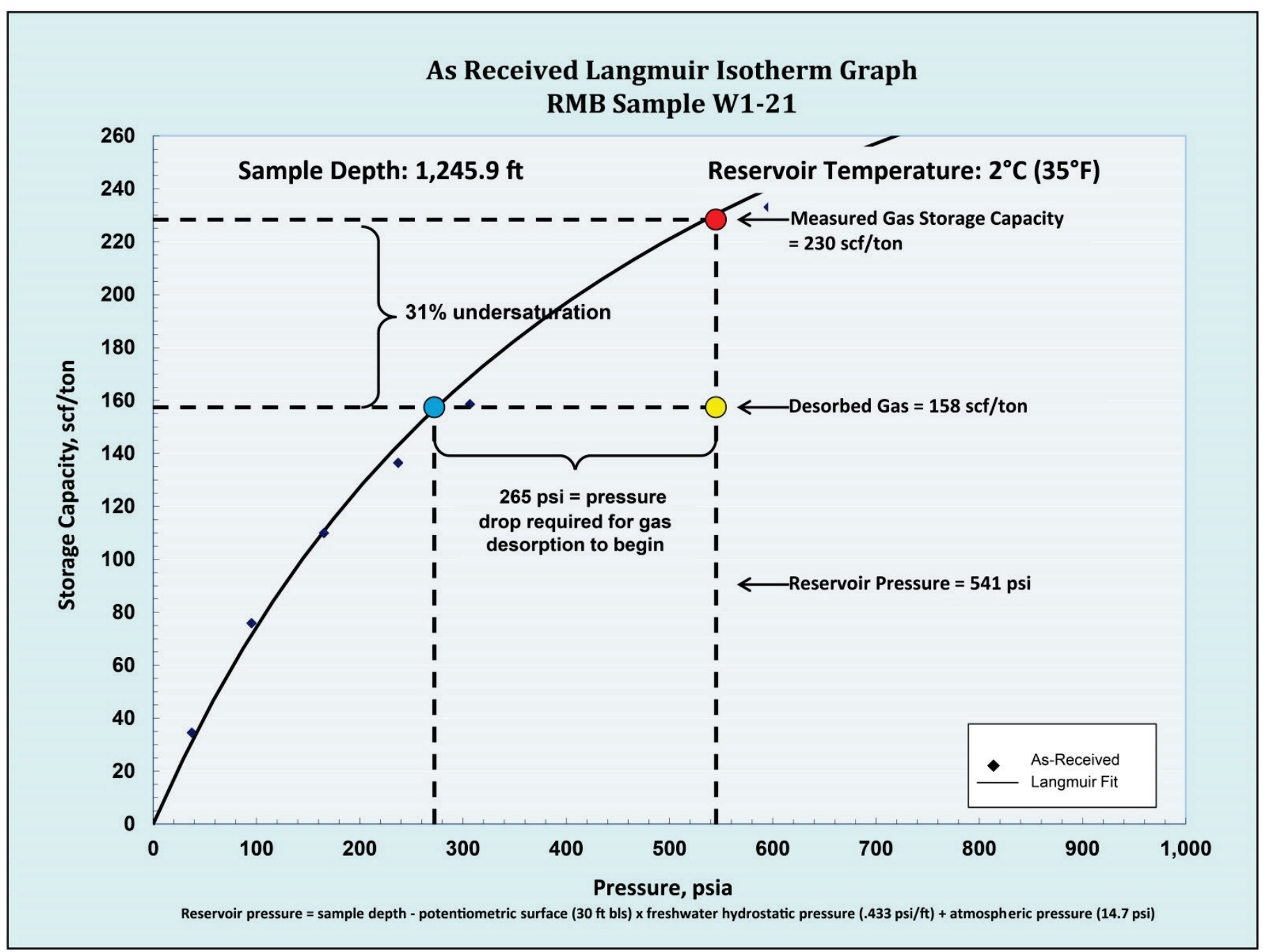

Figure 16. Isotherm chart from well Wainwright-1, desorption canister W1-21, analyzed by RMB Earth Science Consultants, Inc. Abbreviations: scf/ton, standard cubic feet per ton; psi, pounds per square inch; psia, pounds per square inch absolute. 


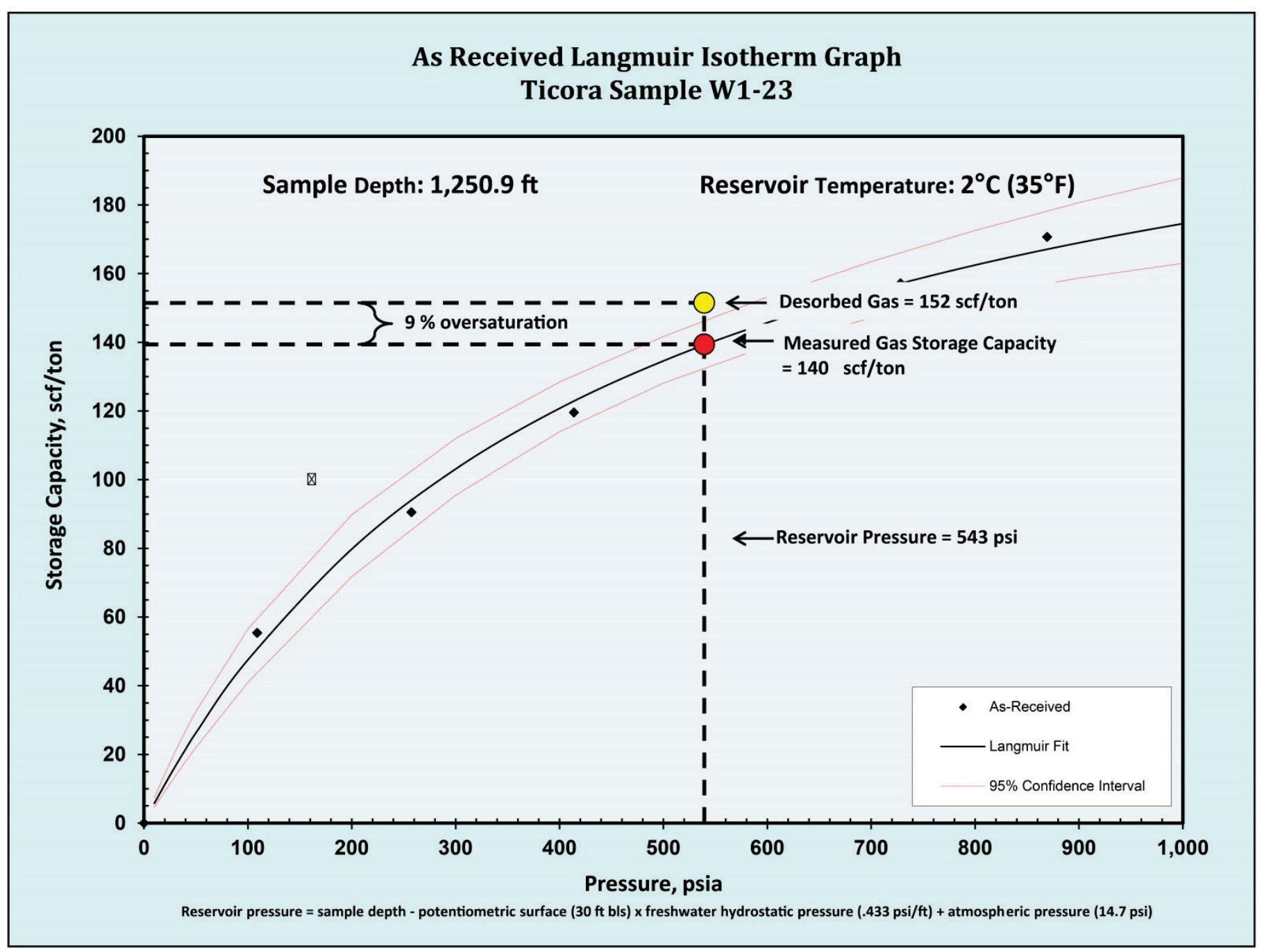

Figure 17. Isotherm chart from well Wainwright-1, desorption canister W1-23, analyzed by Ticora Laboratories. Abbreviations: scf/ton, standard cubic feet per ton; psi, pounds per square inch; psia, pounds per square inch absolute. 


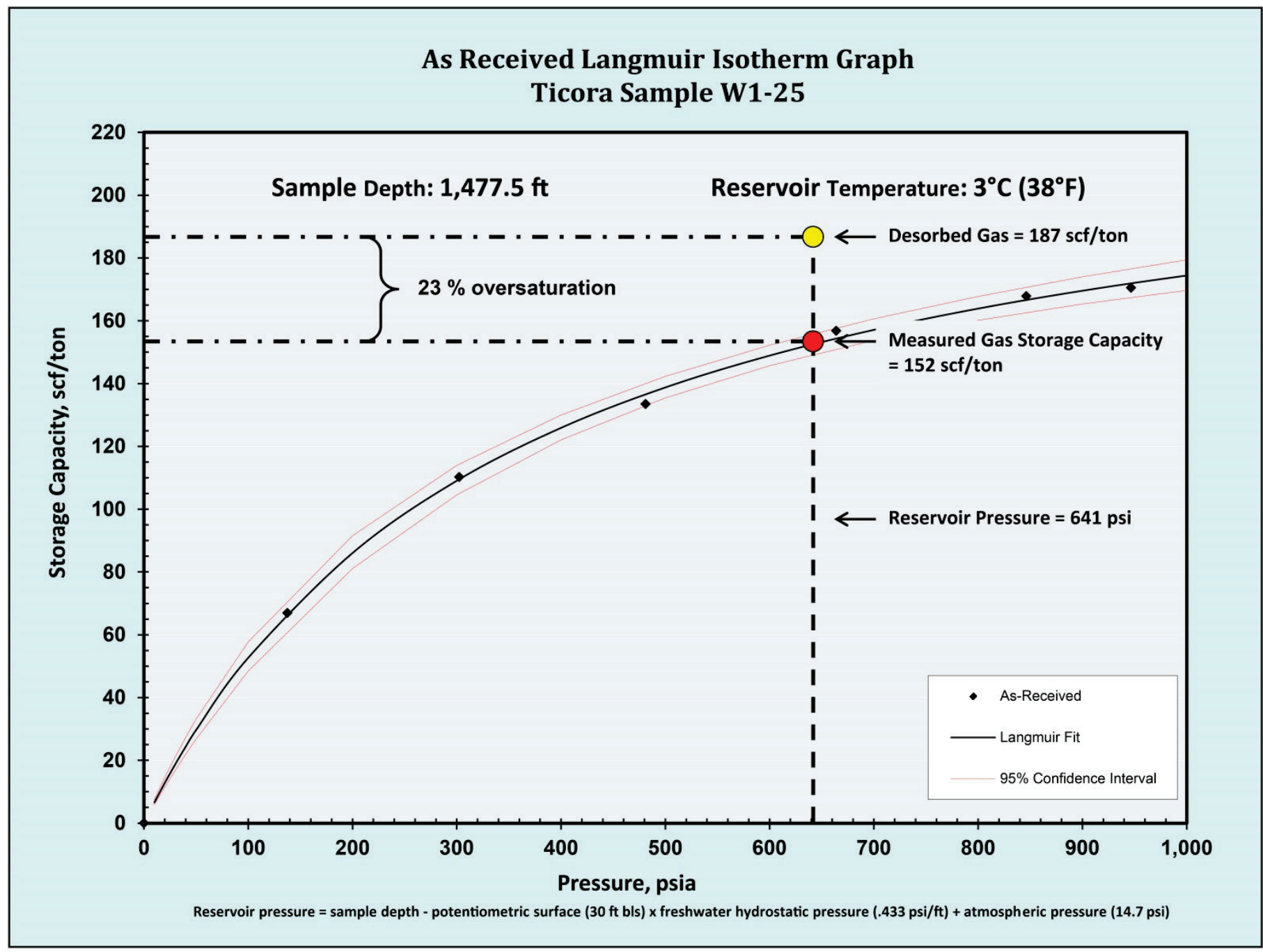

Figure 18. Isotherm chart from well Wainwright-1, desorption canister W1-25, analyzed by Ticora Laboratories. Abbreviations: scf/ton, standard cubic feet per ton; psi, pounds per square inch; psia, pounds per square inch absolute. 


\section{Temperature Log}

A string of thermistors was placed in monitor well Wainwright-2 by UAF personnel on Sept. 19, 2007, and subsurface temperature measurements were collected. A diagram of the temperature readings is shown in figure 19.

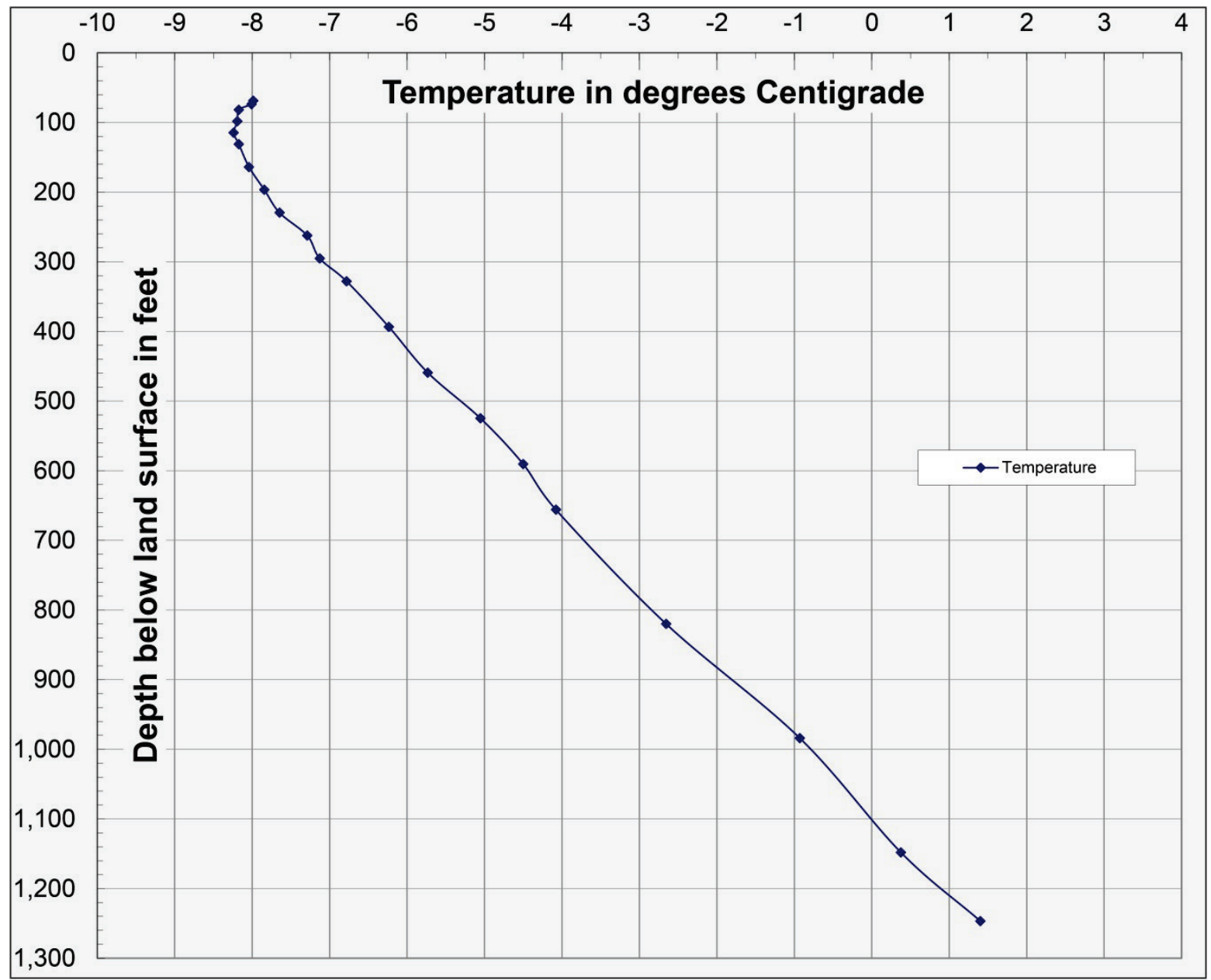

Figure 19. Wainwright-2 temperature log collected by the University of Alaska Fairbanks.

\section{Water Chemistry}

Although water samples were collected from the Wainwright coalbed in the Wainwright-2 well, analytical results suggest that surface water used in the mixing of drilling fluids had not been fully purged from the well prior to sampling. Water samples collected from the Wainwright coalbed during 2009 production testing and presented later in this report (see 2009 water chemistry section) confirmed this contamination. Therefore, analyses of the 2007 water samples are not presented. 


\section{Project Summary and 2008 Plans}

The 2007 drilling and testing effort demonstrated that coalbeds underlying Wainwright, Alaska, contain significant volumes of methane gas and that they are probably at or near full-saturation levels. Preliminary in-place resource calculations for the Wainwright coalbed, based on a 7.5-ft-bed thickness and four core samples with an average desorbed-gas content of $135 \mathrm{scf} /$ ton and an average specific gravity of 1.48 , indicated that each square mile of the coalbed holds approximately 1.18 billion standard cubic feet (bscf) of gas. Based on an energy balance of 1,000 scf of natural gas equaling 7.2 gallons (gal) of diesel fuel, replacing the annual community usage of approximately $1,000,000$ gal of diesel fuel would require approximately 0.14 bscf of gas. These numbers suggested that the Wainwright coalbed, if laterally expansive, contains enough gas to serve as a possible long-term energy source for the community.

After reviewing these results with project partners, a 2008 plan was formulated to (1) drill at least one well at as great a distance from Wainwright-1 as possible to delineate the extent of the Wainwright coalbed and to confirm its gas content, (2) install an array of a single-production well with 4 or 5 monitor wells at the OC ware-yard site to allow preliminary reservoir production testing of the Wainwright coalbed, and (3) drill one of these wells to a depth of approximately $2,500 \mathrm{ft}$ to explore for deeper coalbeds.

On Nov. 13, 2007, results of the 2007 project activities were presented at a meeting of the NSB general Assembly in Barrow, Alaska. In February 2008, after reviewing the 2008 project proposal, the General Assembly voted to commit funds for the project. Subsequently, a not-to-exceed 5-year collaborative agreement between the USGS and NSB was drafted and signed.

A CBNG well-field production class, funded by the ASRC and presented by MHA Petroleum Consultants of Lakewood, Colo., was attended by USGS, BLM, ASRC, and NSB personnel in Golden, Colo., on Apr. 9-10, 2008. The primary purpose of the class was to examine the data collected during 2007 Wainwright drilling activities and design a production test that would effectively define reservoir properties and establish production potential. During discussions, it became evident that a longer test than that outlined in the initial 2008 proposal would be required to adequately establish reservoir properties. Subsequent to the class, 2008 project plans were therefore modified to drill a single delineation well and install a six-well array (fig. 20) to conduct a short production test, test methods, and collect preliminary reservoir data in preparation for a more extensive production test in 2009. In April 2008, permit applications were submitted to AOGCC to drill one delineation well, one production well, and five monitor wells. These wells were permitted by AOGCC as Wainwright-3, $-4,-5,-6,-7,-8$ and -9 . 


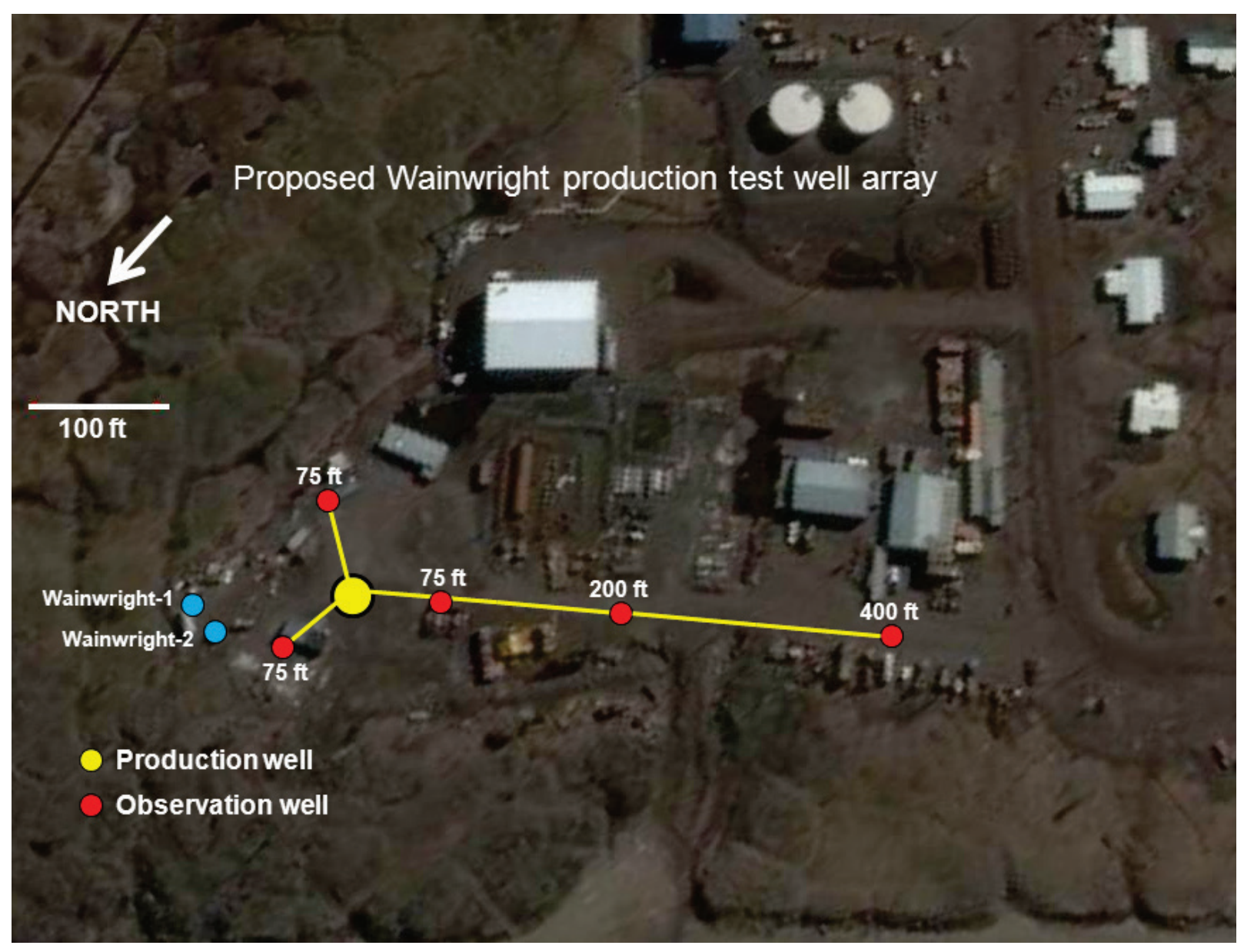

Figure 20. Diagram of proposed Wainwright Olgoonik Corporation ware-yard production-test-well array.

\section{Equipment Transport}

Upon signing the collaborative agreement with the North Slope Borough, the USGS began assembling equipment and purchasing supplies for the upcoming 2008 field season. Because the core rig was inadequate to effectively drill the noncore wells, a larger truck-mounted drilling rig and 3,000 ft of rotary-core drill pipe were transported from Denver to Prudhoe Bay for air transport to Wainwright. PVC monitor-well casing was purchased and shipped to Prudhoe Bay from Manhattan, Kans., and drill mud, cement, well-completion supplies, 6-in. steel pipe, and drums of propylene glycol for use as antifreeze were purchased and shipped to Prudhoe Bay from Fairbanks, Alaska. During transport, $500 \mathrm{ft}$ of the drill pipe was severely damaged leaving 2,500 ft of pipe available for 2008 project use. USGS project personnel arrived in Prudhoe Bay on May 29, 2008, prepared equipment for air transport, and flew to Wainwright on May 31. On June 1-2, six C-130 cargo flights of equipment and supplies were flown from Prudhoe Bay to Wainwright (fig. 21) where it was unloaded, reassembled, and prepared for drilling activities. 


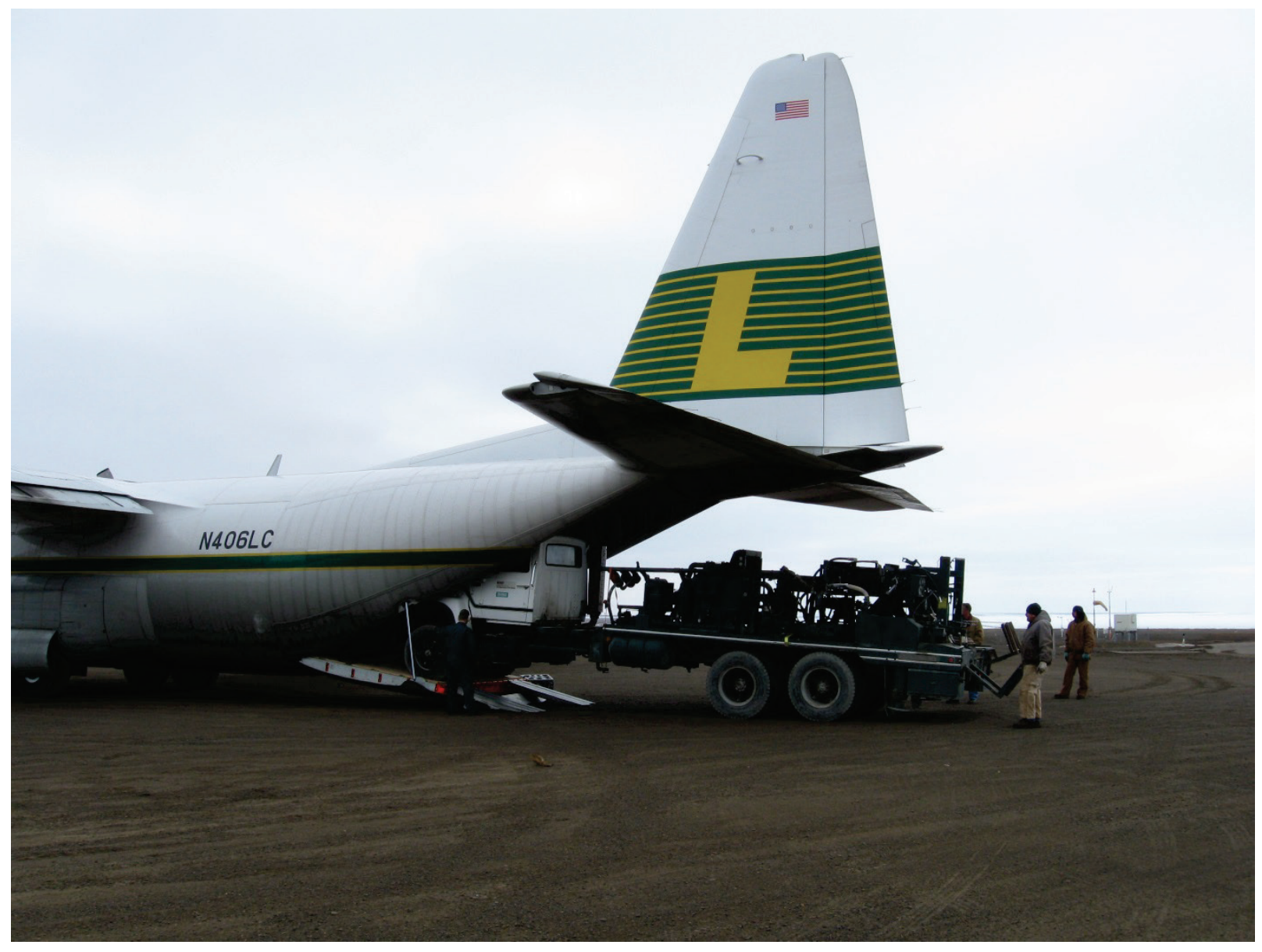

Figure 21. Unloading truck-mounted drill rig from C-130 transport plane in Wainwright, Alaska.

\section{Project Activities}

Initial plans were to begin 2008 activities at the delineation site chosen near the Wainwright landfill approximately 1.9 mi northeast of the Wainwright -1 test well. Upon arrival, however, it was clear that access to the permitted site would not be possible for at least several weeks while remaining snow drifts melted and the area dried. Rather than delay, it was decided to begin drilling at the $\mathrm{OC}$ ware-yard where surface conditions allowed immediate equipment access for the 2,500-ft W-OC1 well (AOGCC Wainwright8 , lat $70^{\circ} 38^{\prime} 37.1^{\prime \prime} \mathrm{N}$., long $160^{\circ} 01^{\prime} 25.9^{\prime \prime} \mathrm{W}$.). Drilling commenced on June 6 . The well was drilled from June 7-13 to a depth of 2,450 ft, and geophysical logs (natural gamma, density, caliper, and resistivity) were collected on June 14. After plugging the bottom $1,185 \mathrm{ft}$ of the hole with abandonment grout, the monitor well was set and completed in the Wainwright coalbed from June 15-17. After filling the monitor well with a propylene glycol/water antifreeze mixture, equipment was moved to the second well site. Monitor well W-OC2 (AOGCC Wainwright-6, lat $70^{\circ} 38^{\prime} 39.0^{\prime \prime} \mathrm{N}$., long $160^{\circ} 01^{\prime} 22.9^{\prime \prime} \mathrm{W}$.) was drilled to a depth of $1,265 \mathrm{ft}$, and a monitor well was set and completed in the Wainwright coalbed from June 18-24. Work began on well W-OC3 (AOGCC Wainwright- 4 , lat $70^{\circ} 38^{\prime} 40.3^{\prime \prime} \mathrm{N}$., long $160^{\circ} 01^{\prime} 21.2^{\prime \prime} \mathrm{W}$. ) on June 25 , but equipment problems on June 27 required the crews to cease operations and return to Denver while 
repair parts were purchased and shipped to Wainwright. Drill-crew personnel returned to Wainwright on July 11 and drilling resumed on July 12 . Monitor well W-OC3 was set and completed on July 14. Monitor well W-OC4 (AOGCC Wainwright-5, lat $70^{\circ} 38^{\prime} 39.5^{\prime \prime} \mathrm{N}$., long $160^{\circ} 01^{\prime} 18.3^{\prime \prime} \mathrm{W}$.) was drilled, set, and completed from July $15-19$. Given the close proximity between wells Wainwright $-1,-2$, and W-OC1 and the excellent correlation of strata between these wells, geophysical logs were not collected from the $\mathrm{W}-\mathrm{OC} 2,-\mathrm{OC} 3$, and -OC4 wells. Due to the extended time required to drill, set, and complete the four monitor wells, a decision was made to forego the fifth monitor well (AOGCC well Wainwright-7). The production well W-OCP (AOGCC Wainwright-3, lat $70^{\circ} 38^{\prime} 39.6^{\prime \prime} \mathrm{N}$., long $160^{\circ} 01^{\prime} 20.8^{\prime \prime} \mathrm{W}$.) was drilled between July $20-28$ to a depth of 1,234 $\mathrm{ft}$. To retain the full thickness of the Wainwright coalbed for testing purposes and to protect the coal reservoir from drilling fluid or cement contamination, it was decided to set production casing $8 \mathrm{ft}$ above, rather than into the coalbed itself. A $65 / 8$-in.-diameter steel casing string was set and cemented to $1,234 \mathrm{ft}$ on July $30-31$ and a 6 -in.-diameter hole was drilled below the casing to a depth of $1,260 \mathrm{ft}$ on Aug. 1. The Wainwright coalbed was penetrated from 1,242 to $1,249.5 \mathrm{ft}$. The coal zone below the casing was underreamed to a 10 -in. diameter from 1,241 to $1,252 \mathrm{ft}$, and the well was cleaned and filled with antifreeze on Aug. 2.

Due to the time spent drilling and installing the production-test-well array, the drilling of the landfill delineation well was delayed until the 2009 field season. The location of drilled wells W-OC1, W-OC2, W-OC3, W-OC4, and W-OCP are shown in figure 22. 


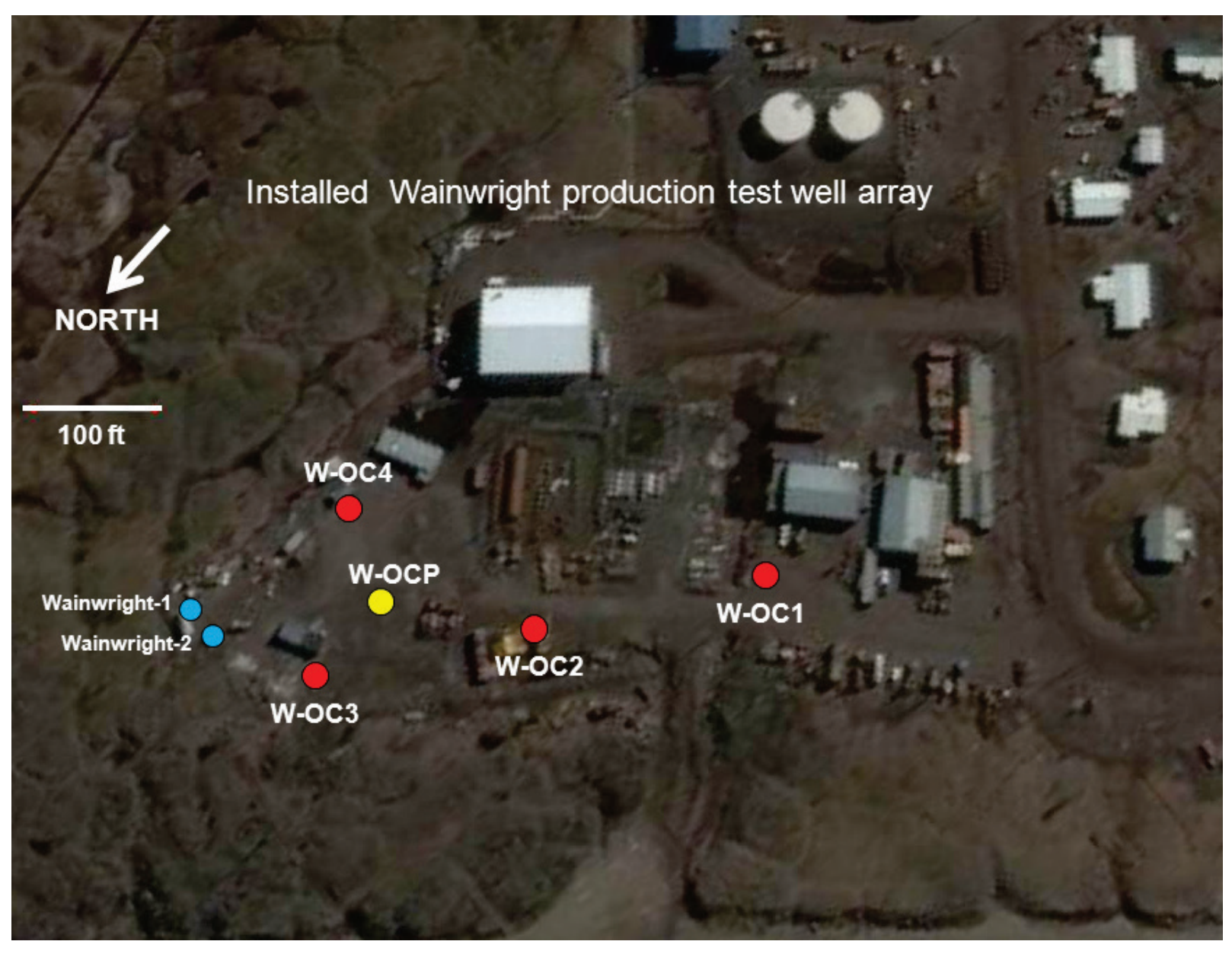

Figure 22. Wainwright Olgoonik Corporation ware-yard production-test-well array.

On Aug. 3, a Grunfos ${ }^{\mathrm{TM}} 4$-in. submersible pump designed for $\mathrm{CBNG}$ use was set to a depth of 1,200 ft, an In Situ ${ }^{\mathrm{TM}}$ pressure transducer was set at $950 \mathrm{ft}$, a gas-water separation tank and flow meters were installed, and a Yaskawa ${ }^{\mathrm{TM}}$ VFD (variablefrequency drive) pump control was connected, programmed, and tested (fig. 22). After programming the monitor-well transducers, a step-drawdown pump test was started on Aug. 4 and continued through the evening of Aug. 8, during which small volumes of methane gas were produced from the well. At that time, a flat-line in recorded pressure suggested that ice might be forming in the wellbore annulus somewhere between the pressure transducer and the submersible pump. To eliminate the possibility of freezing the pump in place, the test was terminated, the well was flooded with water to purge it of methane, and all equipment was removed from the well. The well was then topped with water and allowed to freeze for the winter. 


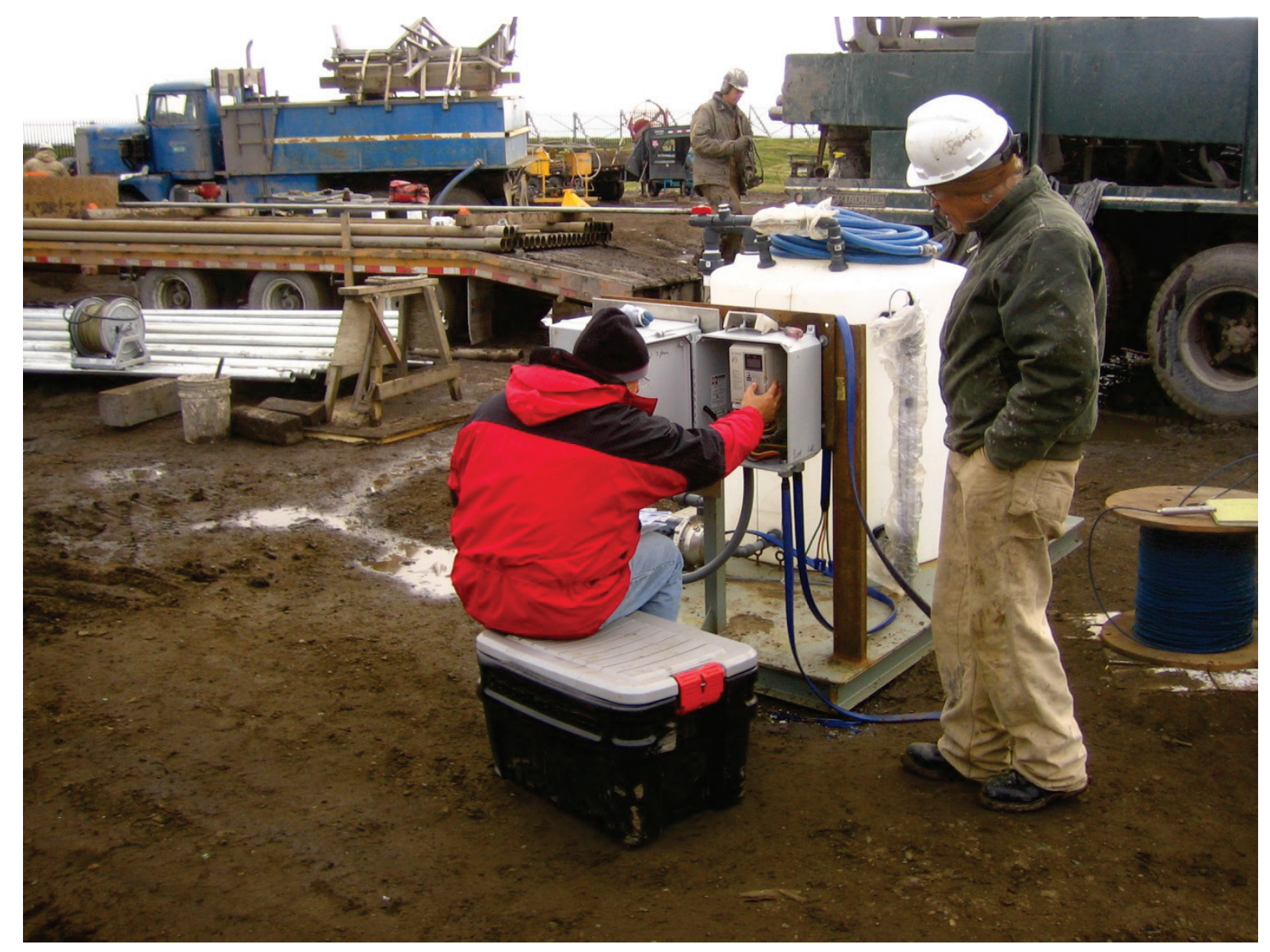

Figure 23. Installing the variable-frequency-drive pump control for production testing of the WOCP (Wainwright-3) well.

After winterizing and packing equipment and installing protective well covers, drill-crew personnel departed Wainwright on Aug. 10. After retrieving monitor-well recovery data, the scientific crew left Wainwright on Aug. 11. Final monitor-well recovery data was retrieved during a trip to Wainwright on Sept. 19.

\section{Project Results}

The majority of the 2008 project focused on drilling and installing the productiontest-well array. However, drilling the 2,450-ft-deep W-OC1 well revealed that no coalbeds of significant thickness underlie the Wainwright coalbed and demonstrated that virtually all gas encountered in the well was related to the penetration of coalbeds. The W-OC1 geophysical and mud-gas logs are shown in plate 2. Mud-gas samples were collected at approximately 100 -ft intervals and sent to Isotech Laboratories, Inc., for compositional and isotopic analyses. Analytical results are shown in table 6 .

Although produced-gas volumes were small, the 4-day production test did confirm that gas could be produced from the Wainwright coalbed and that changes in reservoir pressure could be monitored using the monitor-well array. 
Table 6. Mud-gas analysis from Wainwright well W-OC1 (Wainwright-8).

[Abbreviation: ppm, parts per million; \%, per mil]

\begin{tabular}{|c|c|c|c|c|c|c|c|c|c|c|c|c|c|c|c|c|c|c|c|}
\hline $\begin{array}{l}\text { Well } \\
\text { name }\end{array}$ & $\begin{array}{l}\text { Sample } \\
\text { date }\end{array}$ & $\begin{array}{c}\text { Sample } \\
\text { time }\end{array}$ & $\begin{array}{l}\text { Depth } \\
\text { feet }\end{array}$ & $\begin{array}{c}\text { Gas } \\
\text { units }\end{array}$ & $\begin{array}{c}\mathrm{O}_{2}+\mathrm{Ar} \\
\mathrm{ppm}\end{array}$ & $\begin{array}{l}\mathrm{CO}_{2} \\
\mathrm{ppm}\end{array}$ & $\begin{array}{c}\mathrm{N}_{2} \\
\mathrm{ppm}\end{array}$ & $\begin{array}{c}\text { CO } \\
\text { ppm }\end{array}$ & $\begin{array}{c}\mathrm{C}_{1} \\
\mathrm{ppm}\end{array}$ & $\begin{array}{c}\mathrm{C}_{2} \\
\mathrm{ppm}\end{array}$ & $\begin{array}{l}\mathrm{C}_{2} \mathrm{H}_{4} \\
\mathrm{ppm}\end{array}$ & $\begin{array}{c}\mathrm{C}_{3} \\
\mathrm{ppm}\end{array}$ & $\begin{array}{l}\mathrm{C}_{3} \mathrm{H}_{6} \\
\mathrm{ppm}\end{array}$ & $\begin{array}{c}\mathrm{iC}_{4} \\
\mathrm{ppm}\end{array}$ & $\begin{array}{l}\mathrm{nC}_{4} \\
\mathrm{ppm}\end{array}$ & $\begin{array}{l}\mathrm{iC}_{5} \\
\mathrm{ppm}\end{array}$ & $\begin{array}{l}\mathrm{nC}_{5} \\
\mathrm{ppm}\end{array}$ & $\begin{array}{l}\mathrm{C}_{6+}+ \\
\mathrm{ppm}\end{array}$ & $\begin{array}{c}\delta^{13} \mathrm{C}_{1} \\
\% 0\end{array}$ \\
\hline W-OC1 & $06 / 07 / 08$ & $16: 24$ & 120 & 6 & 219200 & 1300 & 779400 & 0 & 87 & 1 & 0 & 2 & 0 & 0 & 0 & 0 & 0 & 0 & \\
\hline W-OC1 & $06 / 07 / 08$ & $20: 40$ & 200 & 50 & 215700 & 1000 & 778200 & 0 & 5130 & 1 & 0 & 0 & 0 & 0 & 0 & 0 & 0 & 0 & -60.6 \\
\hline W-OC1 & $06 / 08 / 08$ & $14: 55$ & 300 & 40 & 220700 & 500 & 775300 & 0 & 3480 & 1 & 0 & 0 & 0 & 0 & 0 & 0 & 0 & 0 & -59.6 \\
\hline W-OC1 & 06/08/08 & $17: 50$ & 400 & 40 & 218300 & 540 & 776300 & 0 & 4860 & 1 & 1 & 0 & 0 & 0 & 0 & 0 & 0 & 0 & -58.1 \\
\hline W-OC1 & $06 / 08 / 08$ & 22:05 & 510 & 930 & 193800 & 500 & 729100 & 0 & 76600 & 3 & 0 & 0 & 0 & 0 & 0 & 0 & 0 & 0 & -58.5 \\
\hline W-OC1 & 06/09/08 & $2: 13$ & 640 & 105 & 211200 & 510 & 776900 & 0 & 11400 & 2 & 0 & 0 & 0 & 0 & 0 & 0 & 0 & 0 & -56.4 \\
\hline W-OC1 & $06 / 09 / 08$ & $3: 30$ & 700 & 290 & 214500 & 520 & 759500 & 0 & 25500 & 3 & 0 & 0 & 0 & 0 & 0 & 0 & 0 & 0 & -57.4 \\
\hline W-OC1 & 06/09/08 & $6: 23$ & 800 & 90 & 212700 & 540 & 778400 & 0 & 8320 & 2 & 0 & 0 & 0 & 0 & 0 & 0 & 0 & 0 & -55.4 \\
\hline W-OC1 & 06/09/08 & $11: 45$ & 900 & 76 & 211900 & 630 & 779900 & 0 & 7580 & 3 & 0 & 1 & 0 & 0 & 0 & 0 & 0 & 0 & -54.8 \\
\hline W-OC1 & $06 / 09 / 08$ & $21: 51$ & 1000 & 159 & 211200 & 450 & 774800 & 0 & 13500 & 1 & 0 & 0 & 0 & 0 & 0 & 0 & 0 & 0 & -53.4 \\
\hline W-OC1 & $06 / 10 / 08$ & $0: 40$ & 1100 & 173 & 203800 & 450 & 778800 & 0 & 16900 & 5 & 0 & 1 & 0 & 0 & 0 & 0 & 0 & 0 & -54.6 \\
\hline W-OC1 & $06 / 10 / 08$ & $3: 53$ & 1200 & 125 & 207900 & 500 & 779700 & 0 & 11900 & 5 & 0 & 1 & 0 & 0 & 0 & 0 & 0 & 0 & -54.2 \\
\hline W-OC1 & $06 / 10 / 08$ & $7: 47$ & 1300 & 192 & 205300 & 500 & 775800 & 0 & 18400 & 7 & 0 & 2 & 0 & 1 & 1 & 0 & 0 & 0 & -53.9 \\
\hline W-OC1 & $06 / 10 / 08$ & $14: 00$ & 1400 & 120 & 207400 & 620 & 780900 & 0 & 11100 & 4 & 0 & 1 & 0 & 0 & 0 & 0 & 0 & 0 & -53.2 \\
\hline W-OC1 & 06/10/08 & $20: 30$ & 1500 & 194 & 206800 & 480 & 775300 & 0 & 17400 & 4 & 0 & 0 & 0 & 0 & 0 & 0 & 0 & 0 & -53.4 \\
\hline W-OC1 & $06 / 11 / 08$ & $1: 44$ & 1600 & 127 & 208700 & 540 & 778800 & 0 & 12000 & 6 & 0 & 1 & 0 & 0 & 0 & 0 & 0 & 0 & -52.8 \\
\hline W-OC1 & 06/11/08 & $8: 22$ & 1700 & 174 & 205400 & 600 & 778200 & 0 & 15800 & 13 & 0 & 1 & 1 & 0 & 0 & 0 & 0 & 0 & -52.3 \\
\hline W-OC1 & $06 / 12 / 08$ & $15: 00$ & 180 & 180 & 2129 & 440 & 770800 & 0 & 15800 & 81 & 0 & 1 & 0 & 1 & 1 & 1 & 0 & 1 & -49.4 \\
\hline W-OC1 & $06 / 12 / 08$ & $21: 00$ & 19 & 13 & 208 & 480 & 778 & 0 & 122 & 183 & 0 & 33 & 0 & 6 & 4 & 3 & 1 & 4 & -48.3 \\
\hline W-OC1 & 06/13/08 & $1: 39$ & 2000 & 125 & 207800 & 500 & 780100 & 0 & 11400 & 143 & 0 & 35 & 0 & 6 & 4 & 2 & 1 & 3 & -47.6 \\
\hline W-OC1 & $06 / 13 / 08$ & $7: 17$ & 2100 & 154 & 205500 & 310 & 779700 & 0 & 14400 & 75 & 0 & 20 & 0 & 4 & 3 & 2 & 1 & 3 & -47.3 \\
\hline W-OC1 & $06 / 13 / 08$ & $13: 05$ & 2200 & 153 & 207900 & 340 & 778800 & 0 & 12900 & 55 & 0 & 12 & 0 & 3 & 2 & 1 & 0 & 3 & -48.3 \\
\hline W-OC1 & $06 / 13 / 08$ & $19: 18$ & 2300 & 119 & 207300 & 410 & 781800 & 0 & 10400 & 76 & 0 & 11 & 0 & 6 & 5 & 3 & 1 & 4 & -48.1 \\
\hline W-OC1 & $06 / 14 / 08$ & $4: 32$ & 2400 & 113 & 210500 & 280 & 778600 & 0 & 10500 & 82 & 0 & 11 & 0 & 5 & 4 & 3 & 1 & 5 & -47.4 \\
\hline W-OC1 & 06/14/08 & 8:00 & 2450 & 102 & 210800 & 310 & 779300 & 0 & 9480 & 67 & 0 & 11 & 0 & 6 & 5 & 3 & 2 & 18 & -47.0 \\
\hline
\end{tabular}




\section{Plans}

The 2009 field season objectives were to conduct an extended reservoir production test using the installed well array and to drill one or two delineation wells to define the geometry and confirm the gas content of the Wainwright coalbed. In addition to the previously permitted landfill site, two potential delineation sites were chosen approximately $0.5 \mathrm{mi}$ to the southwest of the OC ware-yard wells and permitted through the AOGCC as Wainwright-10 (primary) and Wainwright-11 (alternate).

\section{Project Activities}

\section{Production Testing I}

In an effort to maximize borehole gas-water separation in the 2009 production test, it was recommended by commercial vendors that the submersible pump be placed below, rather than above the Wainwright coalbed as was done in 2008. This would, in theory, allow produced water to flow downward from the coal to the pump while allowing produced gas to separate and flow upward into the production casing. The initial 2009 plan, therefore, was modified to set the drill rig over the W-OCP production well, drill out the ice, and deepen the well to 1,295 ft. Once down-hole equipment was in place and the test started, the rig would be moved from the well and used to drill the delineation wells.

Project personnel arrived in Wainwright on June 7, positioned equipment over WOCP, and cleaned the hole to well bottom on June 8-9. The well was deepened to 1,295 $\mathrm{ft}$ on June 10, and the pump was set at a depth of 1,265 ft on June 11. After installing the pump-control box, pressure transducers, and the water- and gas-flow meters, the production test was started on June 12. On June 13, however, ice began forming in the fluid-production pipe, and slushy ice began to discharge at land surface (fig. 24).

Although produced-fluid icing did not occur during the 4-day test in 2008 (likely due to drilling-induced near-bore warming), it became obvious that a down-hole heat source would be required to prevent ice from forming in the fluid-production pipe. In order to research options and purchase and transport supplies, the test was stopped and the WOCP well was flooded with water. After removing equipment from the well, the drill rig was moved to the Wainwright landfill to drill delineation well Wainwright-9. 


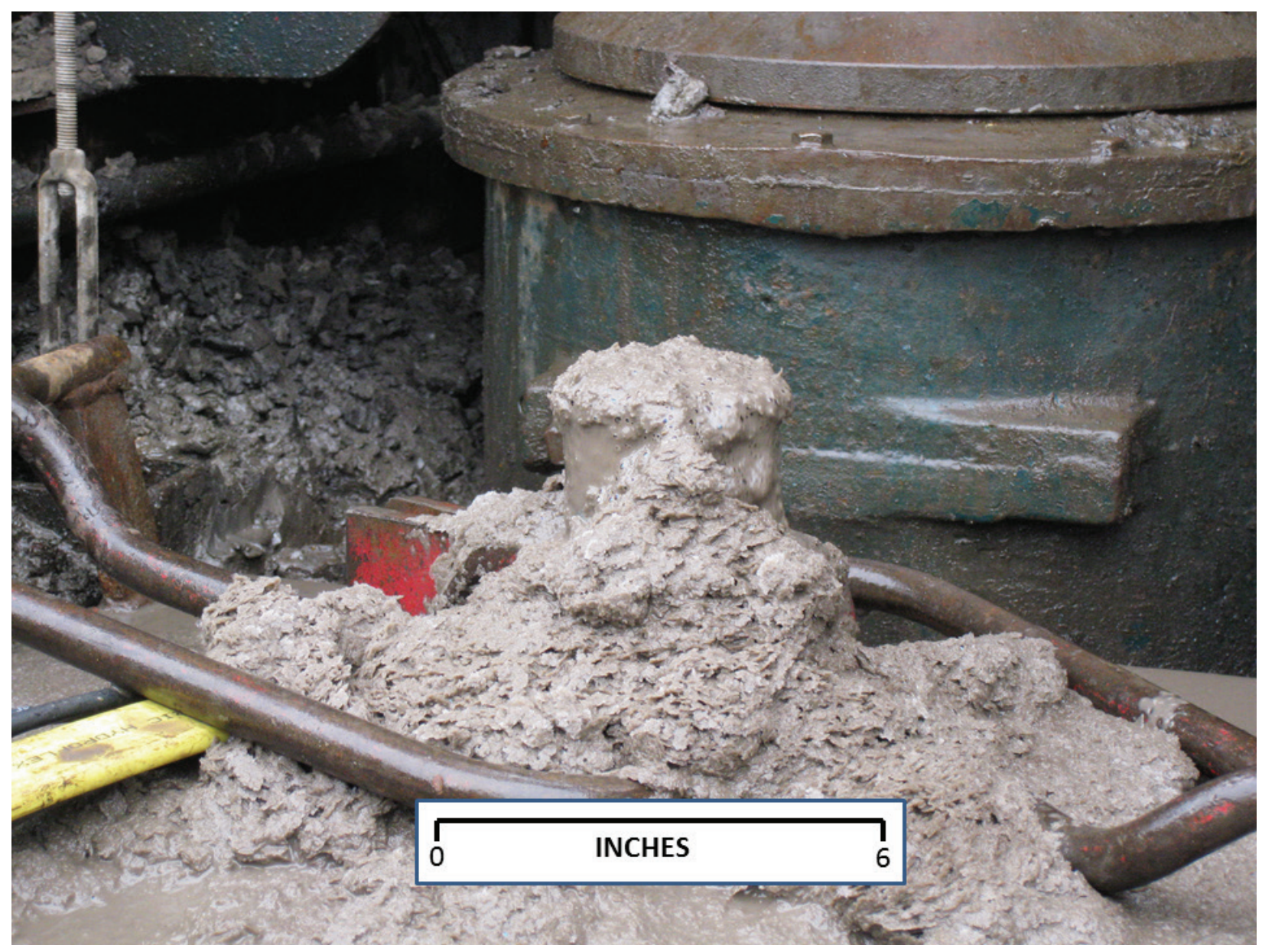

Figure 24. Fluid and ice produced from submersible-pump discharge pipe during initial 2009 production testing in Wainwright well W-OCP.

\section{Well Wainwright-9}

The Wainwright-9 well (lat $70^{\circ} 39^{\prime} 58.6^{\prime \prime} \mathrm{N}$., long $159^{\circ} 58^{\prime} 13.0^{\prime \prime} \mathrm{W}$.) was located near the Wainwright landfill approximately 1.9 mi northeast of the OC ware-yard (for location photo see plate 2). The drilling equipment was moved to the site on June 14, surface casing was set, and open-hole drilling began on June 15. As drilling progressed, correlation of strata between the OC ware-yard and landfill sites suggested that the Wainwright coalbed would be penetrated at a depth of approximately $1,180 \mathrm{ft}$ in the Wainwright-9 well. After open-hole drilling to $1,161 \mathrm{ft}$, continuous core was collected from 1,161 to 1,286 ft on June 18 and 19. The hole was deepened to 1,300 ft and geophysical logs (gamma, caliper, and resistivity) were collected on June 20. Density logs were not collected from the well due to a delay in the transport of the required radioactive source. The Wainwright coalbed was penetrated from 1,167 to $1,174 \mathrm{ft}$ in depth and $6 \mathrm{ft}$ of the coalbed was desorbed for gas content. The well was plugged and abandoned on June 21. 


\section{Wainwright-9 Results}

The geophysical logs for the Wainwright -9 well are shown in plate 2 . The well demonstrated that all coalbeds $>1 \mathrm{ft}$ thick, including the Wainwright coalbed, extend laterally and maintain their general thickness between the OC ware-yard and the Wainwright landfill sites. The apparent dip between the two locations is approximately $40 \mathrm{ft} / \mathrm{mi}$ to the southwest.

Coal cores were desorbed using the methods used at well Wainwright-1 and described in the 2007 project results. Raw desorption values from Wainwright coalbed cores averaged $130 \mathrm{scf} /$ ton, which is slightly less than the $134 \mathrm{scf} /$ ton average of the four Wainwright coalbed cores desorbed from the Wainwright-1 well. Desorption values for the Wainwright-9 coal cores are shown in table 7. Geochemical analyses were conducted on two of the six cores and are shown in table 8 . The average DAF desorption value for these two samples is $191.1 \mathrm{scf} /$ ton (table 9).

Table 7. Raw desorption results from Wainwright-9 coal core samples.

[Abbreviations: ft, foot; g, gram; cc, cubic centimeter; scf/ton, standard cubic feet per ton; DAF, dry-ash-free]

\begin{tabular}{cccccccc}
\hline Well ID & Canister & $\begin{array}{c}\text { Top } \\
\text { (ft) }\end{array}$ & $\begin{array}{c}\text { Bottom } \\
\text { (ft) }\end{array}$ & $\begin{array}{c}\text { Raw } \\
\text { mass } \\
\text { (g) }\end{array}$ & $\begin{array}{c}\text { Lost } \\
\text { gas } \\
\text { (cc) }\end{array}$ & $\begin{array}{c}\text { Measured } \\
\text { gas } \\
\text { (cc) }\end{array}$ & $\begin{array}{c}\text { Raw total } \\
\text { gas } \\
\text { (scf/ton) }\end{array}$ \\
\hline Wainwright-9 & W9-1 & 1168.0 & 1169.3 & 1145 & 409 & 3159 & 99.7 \\
Wainwright-9 & W9-2 & 1169.3 & 1170.5 & 1223 & 572 & 3932 & 117.9 \\
Wainwright-9 & W9-3 & 1170.5 & 1171.5 & 1035 & 386 & 4113 & 139.1 \\
Wainwright-9 & W9-4 & 1171.5 & 1172.5 & 1101 & 781 & 3494 & 124.2 \\
Wainwright-9 & W9-5 & 1172.5 & 1173.5 & 914 & 310 & 3934 & 148.6 \\
Wainwright-9 & W9-6 & 1173.5 & 1174.0 & 623 & 291 & 2638 & 150.4 \\
Wainwright-9 & W9-7 & 1233.4 & 1234.2 & 1580 & 456 & 3855 & 87.3 \\
Wainwright-9 & W9-8 & 1228.0 & 1228.6 & 706 & 191 & 1579 & 80.2 \\
\hline
\end{tabular}


Table 8. As-received geochemical analysis of well Wainwright-9 desorbed-coal samples.

[Abbreviations: ft, feet; wt.-\%, weight percent; lb, pound; Btu, British thermal units; Sub A, subbituminous A]

\begin{tabular}{lrr}
\hline \multicolumn{3}{c}{ Wainwright-9 } \\
\multicolumn{1}{c}{ Sample name } & W9-3 & W9-5 \\
\multicolumn{1}{c}{ Sample depth (ft) } & \multicolumn{1}{c}{1170.5} & \multicolumn{1}{c}{1172.5} \\
\hline Air Dry Loss (wt.-\%) & 16.70 & 16.72 \\
Residual Moisture (wt.-\%) & 2.28 & 1.97 \\
Moisture: (wt-\%) & 18.60 & 18.36 \\
Ash: (wt.-\%) & 8.85 & 3.61 \\
Volatile Matter: (wt.-\%) & 31.69 & 35.18 \\
Fixed Carbon: (wt.-\%) & 40.86 & 42.85 \\
Hydrogen: (wt.-\%) & 6.01 & 6.38 \\
Carbon: (wt.-\%) & 55.44 & 59.45 \\
Nitrogen: (wt.-\%) & 1.60 & 1.63 \\
Sulfur: (wt.-\%) & 0.21 & 0.25 \\
Oxygen (wt.-\%) & 27.89 & 28.68 \\
Heating Value (BTU/lb) & 9839 & 10674 \\
Sulfate Sulfur (wt.-\%) & 0.01 & 0.01 \\
Pyritic Sulfur (wt.-\%) & 0.01 & 0.01 \\
Organic Sulfur (wt.-\%) & 0.19 & 0.23 \\
Equilibrium Moisture (wt.-\%) & 17.18 & 15.62 \\
Lbs Sulfur/Million BTU & 0.21 & 0.23 \\
True Specific Gravity & 1.47 & 1.42 \\
MoistMineralMatterFree BTU & 10881 & 11110 \\
Apparent Rank: & Sub A & Sub A \\
\hline
\end{tabular}


Table 9. Dry-ash-free (DAF) desorption results from two Wainwright-9 coal samples.

[Abbreviations: ft, foot; g, gram; wt-\%, weight percentage; cc, cubic centimeter; scf/ton, standard cubic feet per ton]

\begin{tabular}{cccccccccccc}
\hline Well ID & Canister & $\begin{array}{c}\text { Top } \\
\text { (ft) }\end{array}$ & $\begin{array}{c}\text { Bottom } \\
\text { (ft) }\end{array}$ & $\begin{array}{c}\text { Raw } \\
\text { mass } \\
(\mathbf{g})\end{array}$ & $\begin{array}{c}\text { Moisture } \\
(\text { wt-\%) }\end{array}$ & $\begin{array}{c}\text { Ash } \\
\text { content } \\
(\text { wt-\%) }\end{array}$ & $\begin{array}{c}\text { DAF } \\
\text { mass } \\
\text { (g) }\end{array}$ & $\begin{array}{c}\text { Measured } \\
\text { gas } \\
\text { (cc) }\end{array}$ & $\begin{array}{c}\text { Lost } \\
\text { gas } \\
\text { (cc) }\end{array}$ & $\begin{array}{c}\text { Raw total } \\
\text { gas } \\
\text { (scf/ton) }\end{array}$ & $\begin{array}{c}\text { DAF total } \\
\text { gas } \\
\text { (scf/ton) }\end{array}$ \\
\hline Wainwright-9 & W9-3 & 1170.5 & 1171.5 & 1035 & 18.6 & 8.85 & 751 & 4113 & 386 & 139.1 \\
Wainwright-9 & W9-5 & 1172.5 & 1173.5 & 914 & 18.36 & 3.61 & 713 & 3934 & 310 & 148.6 & 190.4 \\
\hline
\end{tabular}




\section{Production Testing II}

After completing activities at the Wainwright-9 well site, the drilling equipment was moved back to the OC ware-yard on June 22 and reset over the W-OCP production well. On June 23 ice was drilled and flushed from the well, and a 220-volt (V) power supply was installed to run two 1,100-ft heat-trace lines flown in from Anchorage to mitigate produced-fluid icing problems. After filling the well with the propylene glycolwater antifreeze, the submersible pump was set to a depth of 1,254 ft. Both heat-trace lines were attached to the fluid-production pipe and extended to a depth of 1,050 ft. Transducers were programmed on June 24, and the heat-trace lines were powered to allow the well to warm overnight. On June 25, after making final equipment checks, the submersible pump was turned on. Immediately, communication between the productionwell transducers and the surface-control computer was lost and the electronic gas- and water-flow meters quit functioning. Although partial communication was ultimately reestablished with the transducer set directly above the submersible pump, the simultaneous operation of the $480-\mathrm{V}$ submersible pump and the $220-\mathrm{V}$ heat lines had apparently created an electrical surge that irreparably damaged most of the productionwell downhole components. Rather than pull the equipment from the well, the test was restarted using the semioperable bottom transducer to monitor borehole-fluid level and a 5-gal bucket to measure water production rates. After several hours of pumping sediment-laden water and gas, production rates began to slow and eventually ceased altogether. It was now clear that the uncased portion of the hole, which consisted of thin interbeds of sand, silt, and clay (based on unpublished core descriptions from David LePain of the Alaska Division of Geological and Geophysical Surveys and Paul Decker of the Alaska Division of Oil and Gas) had become unstable and was beginning to cave resulting in fine-grained sediment entering and plugging the pump. A decision was made to raise the pump into the production casing with the hope that the caving sediment would separate from the produced water and settle to the bottom of the well. The pump was lifted to a depth of 1,224 ft, but subsequent attempts to restart the pump failed. After filling the well with antifreeze and pulling the pump, it was found that the pump had plugged with sediment to where the motor had overheated and become inoperable. On June 27 , while modifying plans and procuring equipment and supplies, the drill rig was moved approximately $0.5 \mathrm{mi}$ southwest from the OC yard to drill the Wainwright -10 delineation well (lat $70^{\circ} 38^{\prime} 05.8^{\prime \prime} \mathrm{N}$., long $160^{\circ} 01^{\prime} 45.6^{\prime \prime} \mathrm{W}$.; for location see plate 2 ).

\section{Well Wainwright-10}

Based on the geophysical logs from wells Wainwright-1, W-OC1, and Wainwright-9, it was estimated that the Wainwright coalbed would be penetrated at a depth of about $1,300 \mathrm{ft}$ at the Wainwright-10 site. The equipment was set up and, after setting surface casing to $92 \mathrm{ft}$, an open hole was drilled to 1,300 ft on June 29-30 (fig. 25). Cores were collected from 1,300 to 1,320 ft on July 1, the hole was deepened to $1,360 \mathrm{ft}$, and geophysical logs (gamma, density, caliper, and resistivity) were run on July 2. A water sample was collected at a depth of 1,160 ft from a 100-ft-thick sandstone on July 3, and the well was plugged and abandoned on July 4. A total of $8.5 \mathrm{ft}$ of coal core, all of which was desorbed, was collected from Wainwright-10. The Wainwright coalbed was penetrated at depths between 1,300.4 and 1,307.8 ft. 


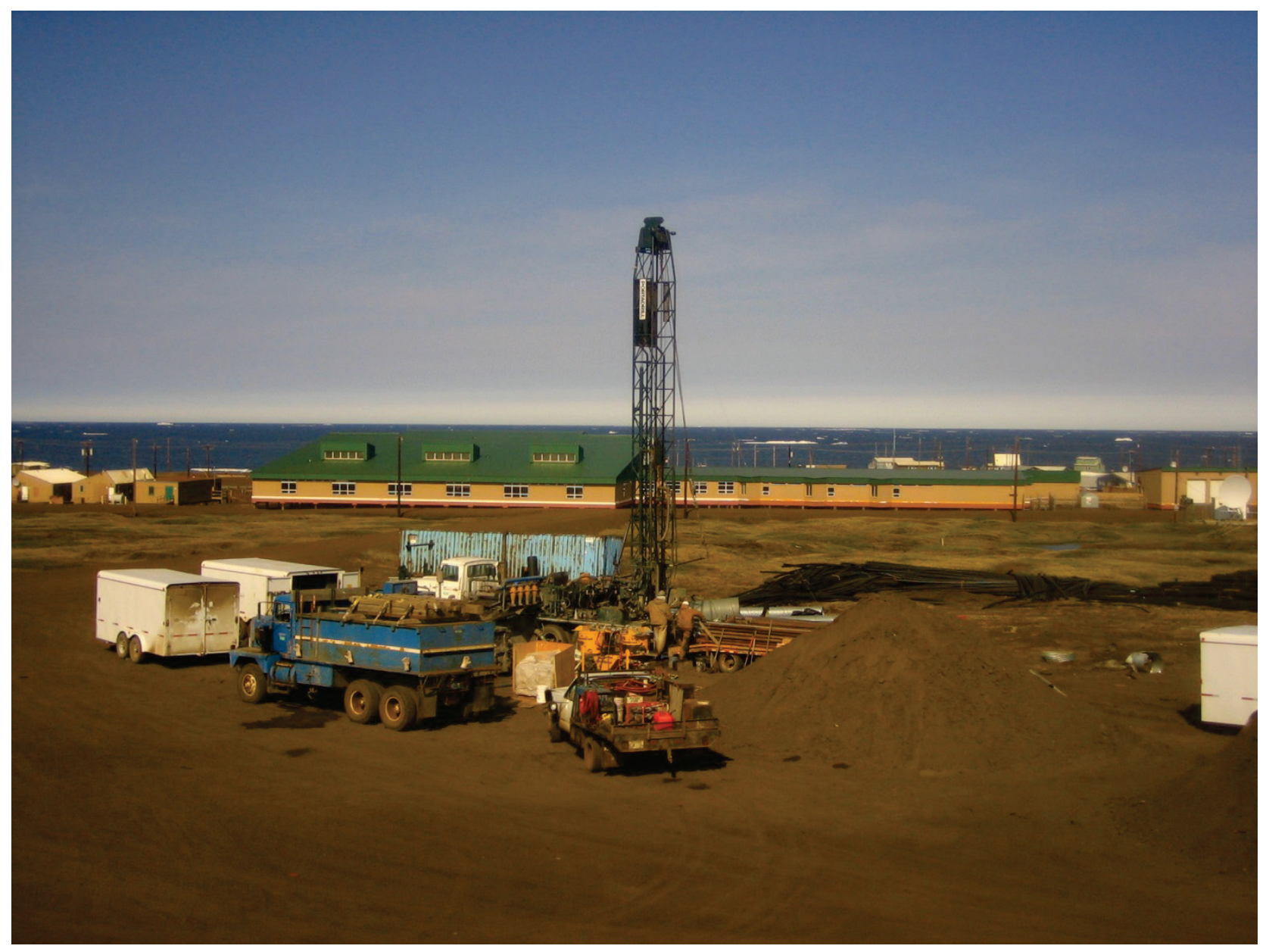

Figure 25. Drilling the Wainwright-10 delineation well.

\section{Wainwright-10 Results}

The geophysical logs from Wainwright-10 are shown in plate 2 . The well demonstrated that the Wainwright coalbed extends and maintains its general thickness to this location and that coalbeds $>1 \mathrm{ft}$ thick and major sand bodies are continuous between the three well sites (for stratigraphic correlations between well sites see Clark and others, 2010). Triangulating the elevation of the top of the Wainwright coalbed in wells Wainwright-1, Wainwright-9, and Wainwright-10 indicates a structural dip of about 100 $\mathrm{ft} / \mathrm{mi}$ to the southeast.

Coal cores were desorbed using the methods as described earlier. Raw desorption values for the $7.4 \mathrm{ft}$ of coal collected from the Wainwright coalbed (table 9) averaged $145.9 \mathrm{scf} /$ ton, which is about 10 percent higher than the bed's average raw desorption values from wells Wainwright-1 (134.7 scf/ton) and Wainwright-9 (130.0 scf/ton). Geochemical analyses were conducted on two of the seven core samples and are shown in table 11. The average DAF desorption values for these two samples (table 12) is 192.2 scf/ton, which is very similar to the DAF values from the Wainwright-1 (189.9 scf/ton) and Wainwright-9 (191.1 scf/ton) cores. 
Table 10. Raw desorption results from Wainwright-10 coal core samples.

[Abbreviations: ft, foot; g, grams; cc, cubic centimeters; scf/ton, standard cubic feet per ton]

\begin{tabular}{cccccccc}
\hline Well ID & Canister & $\begin{array}{c}\text { Top } \\
\text { (ft) }\end{array}$ & $\begin{array}{c}\text { Bottom } \\
\text { (ft) }\end{array}$ & $\begin{array}{c}\text { Raw } \\
\text { mass } \\
\text { (g) }\end{array}$ & $\begin{array}{c}\text { Lost } \\
\text { gas } \\
\text { (cc) }\end{array}$ & $\begin{array}{c}\text { Measured } \\
\text { gas } \\
\text { (cc) }\end{array}$ & $\begin{array}{c}\text { Raw total } \\
\text { gas } \\
\text { (scf/ton) }\end{array}$ \\
\hline Wainwright-10 & W10-1 & 1300.4 & 1301.5 & 1087 & 301 & 4843 & 151.42 \\
Wainwright-10 & W10-2 & 1301.5 & 1302.5 & 1063 & 248 & 4751 & 150.49 \\
Wainwright-10 & W10-3 & 1302.5 & 1303.6 & 1058 & 234 & 4178 & 133.45 \\
Wainwright-10 & W10-4 & 1303.6 & 1304.6 & 1092 & 390 & 4503 & 143.38 \\
Wainwright-10 & W10-5 & 1304.6 & 1305.7 & 1191 & 381 & 4835 & 140.14 \\
Wainwright-10 & W10-6 & 1305.7 & 1306.8 & 1105 & 281 & 4929 & 150.86 \\
Wainwright-10 & W10-7 & 1306.8 & 1307.8 & 1026 & 239 & 4616 & 151.42 \\
Wainwright-10 & W10-8 & 1311.0 & 1312.1 & 1354 & 476 & 3787 & 100.76 \\
\hline
\end{tabular}

Table 11. As-received geochemical analysis for two Wainwright-10 desorbed-coal samples.

[Abbreviations: ft, feet; wt.-\%, weight percent, lb, pound; Btu, British thermal units; Sub A, subbituminous A]

\begin{tabular}{lcc}
\hline \multicolumn{3}{c}{ Wainwright-10 } \\
\hline \multicolumn{1}{c}{ Sample name } & W10-2 & W10-6 \\
Sample depth (ft) & $\mathbf{1 3 0 1 . 5}$ & \multicolumn{1}{c}{1305.7} \\
\hline Air Dry Loss & 16.43 & 16.12 \\
Residual Moisture & 2.17 & 2.11 \\
Moisture & 18.24 & 17.89 \\
Ash & 1.43 & 5.54 \\
Volatile Matter & 36.58 & 34.63 \\
Fixed Carbon & 43.75 & 41.94 \\
Hydrogen & 6.45 & 6.08 \\
Carbon & 61.76 & 58.67 \\
Nitrogen & 1.58 & 1.54 \\
Sulfur & 0.23 & 0.19 \\
Oxygen & 28.55 & 27.98 \\
Heating Value (Btu/lb) & 11080 & 10357 \\
Sulfate Sulfur & 0.01 & 0.01 \\
Pyritic Sulfur & 0.01 & 0.02 \\
Organic Sulfur & 0.21 & 0.16 \\
Free Swelling Index & 0.0 & 0.0 \\
Equilibrium Moisture & 15.72 & 15.37 \\
Lbs Sulfur/Million Btu & 0.21 & 0.18 \\
True Specific Gravity & 1.44 & 1.43 \\
MoistMineralMatterFreeBtu & 11256.6 & 11018.3 \\
Apparent Rank: & Sub A & Sub A \\
\hline
\end{tabular}


Table 12. Dry-ash-free (DAF) desorption results from two Wainwright-10 coal samples.

[Abbreviations: ft, foot; g, gram; wt-\%, weight percentage; cc, cubic centimeter; scf/ton, standard cubic feet per ton]

\begin{tabular}{|c|c|c|c|c|c|c|c|c|c|c|c|c|}
\hline Well ID & Canister & $\begin{array}{c}\text { Report } \\
\text { name }\end{array}$ & $\begin{array}{c}\text { Top } \\
\text { (ft) }\end{array}$ & $\begin{array}{c}\text { Bottom } \\
\text { (ft) }\end{array}$ & $\begin{array}{c}\text { Raw } \\
\text { mass } \\
(\mathrm{g})\end{array}$ & $\begin{array}{c}\text { Moisture } \\
\text { (wt-\%) }\end{array}$ & $\begin{array}{c}\text { Ash } \\
\text { content } \\
\text { (wt-\%) }\end{array}$ & $\begin{array}{l}\text { DAF } \\
\text { mass } \\
(\mathrm{g})\end{array}$ & $\begin{array}{c}\text { Measured } \\
\text { gas } \\
\text { (cc) }\end{array}$ & $\begin{array}{l}\text { Lost } \\
\text { gas } \\
\text { (cc) }\end{array}$ & $\begin{array}{c}\text { Raw total } \\
\text { gas } \\
\text { (scf/ton) }\end{array}$ & $\begin{array}{c}\text { DAF total } \\
\text { gas } \\
\text { (scf/ton) }\end{array}$ \\
\hline Wainwright-10 & W10-2 & W10-2 & 1301.5 & 1302.5 & 1063 & 18.24 & 1.43 & 854 & 4751 & 248 & 150.49 & 187.35 \\
\hline Wainwright-10 & W10-6 & W10-6 & 1305.7 & 1306.8 & 1105 & 17.89 & 5.54 & 846 & 4929 & 281 & 150.86 & 197.03 \\
\hline
\end{tabular}




\section{Production Testing III}

After completing work at the Wainwright-10 site, drilling equipment was moved back to the OC yard and repositioned over the $\mathrm{W}-\mathrm{OCP}$ production well. Before resetting the pump, an attempt was made to run a caliper log in the bottom of the well to determine the extent of sloughing that had occurred. However, the caliper probe could not be lowered below the bottom of the 6-in. casing $(1,234 \mathrm{ft})$ due to borehole blockage, thus confirming that the uncased portion of the well had caved. Flushing and stabilizing the uncased portion of the well with drilling mud was again possible, but it was now clear that the unstable sediments would continue caving once drill mud was removed from the well and production testing resumed. Although a coarse-sand or gravel filter pack might have been placed in the uncased portion of the well in an attempt to stabilize the bore and filter sediment from the produced water, there were inadequate quantities of such material available in Wainwright. Therefore, the decision was made to set the pump inside the casing rather than in the uncased portion of the well and attempt to produce gas and water through the sloughed sediment.

The submersible pump was set to a depth of 1,220 ft on July 5. The two heat-trace lines were turned on to warm the well prior to test startup, but after an hour, one of the lines shorted out. After monitoring borehole temperature for 16 hours, it was evident that a single heat line was not capable of keeping borehole temperatures elevated above the fluid-freezing point. A replacement heat line was therefore ordered and shipped from Anchorage, arriving on July 7. On July 8, after installing the replacement line, the production test was restarted. Testing continued through July 9 with small amounts of water and gas produced; however, the fluid remained silt-laden and production rates slowly diminished. On July 10 the test was stopped, the well was filled with antifreeze, and the pump was again pulled from the well and cleaned.

In a final attempt to remove sediment from the produced water, approximately 3 cubic feet of gravel was sifted from a nearby sediment pile and mixed with 3 cubic feet of silica sand remaining from 2008 project activities. This material was then placed on top of the caved silt through a 2 -in. tremie pipe. Although much of the material bridged in the pipe during installation, the material that was placed provided a filter inside the 6-in. casing from a depth of 1,225 to $1,235 \mathrm{ft}$. The submersible pump was set to a depth of $1,094 \mathrm{ft}$ on July 11, and a transducer was set below the pump to monitor borehole-fluid level. After repairing another heat-line short, the production test was restarted on July 12. Drill-crew personnel departed Wainwright on July 14 leaving a two-person scientific crew to monitor the test. Although complications were encountered, testing continued from July 12 through Aug. 18 during which produced-water and gas samples were collected. Production testing was terminated on Aug. 19, and the well was filled with antifreeze. Drill-crew personnel returned to Wainwright on Aug. 23 to remove equipment from the well and to clean, winterize, and store it for 2010 project use.

\section{Gas Analyses}

A desorbed-gas sample was collected for compositional and isotopic analysis from one desorption canister from both the Wainwright-9 and Wainwright-10 coal cores. As with the gas samples desorbed in 2007, the $\mathrm{O}_{2}$ present in the as-received samples is assumed to be the result of atmospheric contamination, and the analytical results have 
therefore been normalized to an air-free basis. Additionally, a produced-gas sample was collected from the $\mathrm{W}-\mathrm{OCP}$ well during production testing. This sample was collected directly from the gas-production line where gas was allowed to flow through the sample canister for several minutes before the sample was taken. The resulting as-received sample contained only 0.24 percent $\mathrm{O}_{2}$ confirming that the vast majority, if not all, $\mathrm{O}_{2}$ present in the desorbed-gas samples was, in fact, due to atmospheric contamination. Both the as-received and normalized gas composition analyses are shown in table 13. The isotopic analysis from the three as-received samples is shown in table 14. 
Table 13. Compositional analysis of gas samples collected during 2009 season.

[Abbreviations: \%o, per mil, BTU, British thermal units]

\begin{tabular}{|c|c|c|c|c|c|c|c|c|c|c|c|c|c|c|c|c|c|}
\hline \multicolumn{18}{|c|}{ Gas composition as-received } \\
\hline Well name & Sample name & Sample depth & $\begin{array}{c}\mathrm{He} \\
\%\end{array}$ & $\begin{array}{l}\mathrm{H}_{2} \\
\%\end{array}$ & $\begin{array}{l}\mathrm{Ar} \\
\%\end{array}$ & $\begin{array}{l}\mathrm{O}_{2} \\
\%\end{array}$ & $\begin{array}{c}\mathrm{CO}_{2} \\
\%\end{array}$ & $\begin{array}{l}\mathrm{N}_{2} \\
\%\end{array}$ & $\begin{array}{l}\mathrm{CO} \\
\%\end{array}$ & $\begin{array}{l}\mathrm{C}_{1} \\
\%\end{array}$ & $\begin{array}{l}\mathrm{C}_{2} \\
\%\end{array}$ & $\begin{array}{l}\mathrm{C}_{3} \\
\%\end{array}$ & $\begin{array}{c}\mathrm{iC}_{4} \\
\%\end{array}$ & $\begin{array}{c}\mathrm{nC}_{4} \\
\%\end{array}$ & $\begin{array}{l}\mathrm{iC}_{5} \\
\%\end{array}$ & $\begin{array}{c}\mathrm{nC}_{5} \\
\%\end{array}$ & $\begin{array}{c}\mathrm{C}_{6}+ \\
\%\end{array}$ \\
\hline Wainwright-9 & W9-4 & 1171.5 & 0 & 0.00 & 0.34 & 7.57 & 0.05 & 28.28 & 0.00 & 63.75 & 0.01 & 0.00 & 0.00 & 0.00 & 0.00 & 0.00 & 0.00 \\
\hline Wainwright-10 & W10-3 & 1302.5 & 0 & 0.00 & 0.07 & 1.53 & 0.08 & 5.73 & 0.00 & 92.58 & 0.01 & 0.00 & 0.00 & 0.00 & 0.00 & 0.00 & 0.00 \\
\hline W-OCP & W-OCP & 1250.0 & 0.0037 & 0.06 & 0.01 & 0.24 & 1.54 & 0.95 & 0.00 & 97.18 & 0.01 & 0.00 & 0.00 & 0.00 & 0.00 & 0.00 & 0.00 \\
\hline \multicolumn{18}{|c|}{ Gas composition with atmospheric contamination removed } \\
\hline Well Name & Sample name & Sample depth & $\begin{array}{c}\mathrm{He} \\
\%\end{array}$ & $\begin{array}{l}\mathrm{H}_{2} \\
\%\end{array}$ & $\begin{array}{l}\mathrm{Ar} \\
\%\end{array}$ & $\begin{array}{l}\mathrm{O}_{2} \\
\%\end{array}$ & $\begin{array}{c}\mathrm{CO}_{2} \\
\%\end{array}$ & $\begin{array}{l}\mathrm{N}_{2} \\
\%\end{array}$ & $\begin{array}{c}\mathrm{CO} \\
\%\end{array}$ & $\begin{array}{l}\mathbf{C}_{1} \\
\%\end{array}$ & $\begin{array}{l}\mathrm{C}_{2} \\
\%\end{array}$ & $\begin{array}{l}\mathrm{C}_{3} \\
\%\end{array}$ & $\begin{array}{c}\mathrm{iC}_{4} \\
\%\end{array}$ & $\begin{array}{c}\mathrm{nC}_{4} \\
\%\end{array}$ & $\begin{array}{l}\mathrm{iC}_{5} \\
\%\end{array}$ & $\begin{array}{c}\mathrm{nC}_{5} \\
\%\end{array}$ & $\begin{array}{c}\mathrm{C}_{6}+ \\
\%\end{array}$ \\
\hline Wainwright-9 & W9-4 & 1171.5 & 0 & 0.00 & 0.00 & 0.00 & 0.04 & 0.07 & 0.00 & 99.90 & 0.01 & 0.00 & 0.00 & 0.00 & 0.00 & 0.00 & 0.00 \\
\hline Wainwright-10 & W10-3 & 1302.5 & 0 & 0.00 & 0.00 & 0.00 & 0.07 & 0.03 & 0.00 & 99.90 & 0.01 & 0.00 & 0.00 & 0.00 & 0.00 & 0.00 & 0.00 \\
\hline W-OCP & W-OCP & 1250.0 & 0.0037 & 0.06 & 0.00 & 0.00 & 1.54 & 0.05 & 0.00 & 98.35 & 0.01 & 0.00 & 0.00 & 0.00 & 0.00 & 0.00 & 0.00 \\
\hline \multicolumn{18}{|c|}{ Gas composition with atmospheric contamination removed } \\
\hline Well Name & Sample name & Sample depth & $\begin{array}{c}\delta^{13} C_{1} \\
\% 0\end{array}$ & $\begin{array}{c}\delta \mathrm{DC}_{1} \\
\% 0\end{array}$ & & $\begin{array}{l}\text { ecific } \\
\text { avity }\end{array}$ & BTU & & & & & & & & & & \\
\hline Wainwright-9 & W9-4 & 1171.5 & -53.53 & -256.7 & & .716 & 645 & & & & & & & & & & \\
\hline Wainwright-10 & W10-3 & 1302.5 & -53.18 & -258.3 & & .588 & 938 & & & & & & & & & & \\
\hline W-OCP & W-OCP & 1250.0 & -52.20 & -254.2 & & .574 & 986 & & & & & & & & & & \\
\hline
\end{tabular}


Table 14. As-received isotopic analyses of gas samples collected during 2009 season.

[Abbreviations: Btu, British thermal units; \%o, per mil]

\begin{tabular}{llccccc}
\hline \multicolumn{1}{c}{ Well name } & Sample name & Sample depth & $\begin{array}{c}\boldsymbol{\delta}^{13} \mathbf{C}_{1} \\
\% 0\end{array}$ & $\begin{array}{c}\delta \mathbf{\delta C}_{1} \\
\% 0\end{array}$ & $\begin{array}{c}\text { Specific } \\
\text { Gravity }\end{array}$ & BTU \\
\hline Wainwright-9 & W9-4 & 1171.5 & -53.53 & -256.7 & 0.716 & 645 \\
Wainwright-10 & W10-3 & 1302.5 & -53.18 & -258.3 & 0.588 & 938 \\
W-OCP & W-OCP & 1250 & -52.20 & -254.2 & 0.574 & 986 \\
\hline
\end{tabular}

\section{Water Chemistry}

Water-quality samples were collected on July 3 from the Wainwright-10 well in a 100-ft-thick sandstone located $200 \mathrm{ft}$ above the Wainwright coalbed, and on Aug. 1 from the Wainwright coalbed during production testing of the W-OCP well. The Wainwright10 sandstone was isolated by straddle packers placed at 1,140 and 1,180 ft and a slotted screen placed between the packers. After developing the well for 10 hours using air-lift methods and allowing it to recover for an additional 4 hours, water samples were collected from the bottom of the well using a Teflon bailer. The Wainwright coalbedwater sample was collected from the W-OCP well directly from the production-fluid discharge line after several days of continuous fluid production. Filtered samples were refrigerated and sent to the University of Arizona for anion and cation analysis. Analytical results show typical biogenic CBNG-water characteristics in which sodium $(\mathrm{Na})$, chloride $(\mathrm{Cl})$, and bicarbonate $\left(\mathrm{HCO}_{3}\right)$ levels are elevated and sulfate $\left(\mathrm{SO}_{4}\right)$, calcium $(\mathrm{Ca})$, and magnesium $(\mathrm{Mg})$ levels are depleted (Van Vost, 2003). Total dissolved solids (TDS), which are a measure of the total dissolved ions in solution, were determined by summing the measured individual ion concentrations. Doing so gave TDS values of 10,027 milligrams/liter (mg/L) for the Wainwright-10 sandstone sample and $16,304 \mathrm{mg} / \mathrm{L}$ for the Wainwright coalbed sample. According to the Water Quality Association "Glossary of Salt Water" (www.wqa.org), these values would be considered highly brackish $(5,000-15,000 \mathrm{mg} / \mathrm{L})$ to saline $(15,000-30,000 \mathrm{mg} / \mathrm{L})$ in nature. For comparison, sea water has a typical TDS value of between 30,000 and 40,000 mg/L according to the same glossary. Water chemistry analytical results are shown in table 15 .

Table 15. Chemical analysis of water samples collected from a sandstone in well Wainwright-10 and the Wainwright coalbed in well W-OCP.

[Abbreviations: mg/L, milligrams per liter; TDS, total dissolved solids]

\begin{tabular}{lccccccccc}
\hline & $\begin{array}{c}\mathrm{TDS} \\
\mathrm{mg} / \mathrm{L}\end{array}$ & $\begin{array}{c}\mathrm{HCO}_{3} \\
\mathrm{mg} / \mathrm{L}\end{array}$ & $\begin{array}{c}\mathrm{Cl} \\
\mathrm{mg} / \mathrm{L}\end{array}$ & $\begin{array}{c}\mathrm{Br} \\
\mathrm{mg} / \mathrm{L}\end{array}$ & $\begin{array}{c}\mathrm{NO}_{3} \\
\mathrm{mg} / \mathrm{L}\end{array}$ & $\begin{array}{c}\mathrm{SO}_{4} \\
\mathrm{mg} / \mathrm{L}\end{array}$ & $\begin{array}{c}\mathrm{Ca} \\
\mathrm{mg} / \mathrm{L}\end{array}$ & $\begin{array}{c}\mathrm{Mg} \\
\mathrm{mg} / \mathrm{L}\end{array}$ & $\begin{array}{c}\mathrm{Na} \\
\mathrm{mg} / \mathrm{L}\end{array}$ \\
\hline W-0CP & 16304 & 1890 & 8350.80 & 0.00 & 37.69 & 0.00 & 24.50 & 21.02 & 5912.58 \\
Wainwright-10 & 10027 & 1631 & 4638.09 & 0.74 & 20.20 & 10.62 & 12.12 & 10.45 & 3668.08 \\
\hline & $\mathrm{K}$ & $\mathrm{Sr}$ & $\mathrm{Si}$ & $\mathbf{B}$ & $\mathrm{Mn}$ & $\mathrm{Fe}$ & $\mathrm{Zn}$ & $\mathrm{Ba}$ & $\mathrm{Li}$ \\
& $\mathrm{mg} / \mathrm{L}$ & $\mathrm{mg} / \mathrm{L}$ & $\mathrm{mg} / \mathrm{L}$ & $\mathrm{mg} / \mathrm{L}$ & $\mathrm{mg} / \mathrm{L}$ & $\mathrm{mg} / \mathrm{L}$ & $\mathrm{mg} / \mathrm{L}$ & $\mathrm{mg} / \mathrm{L}$ & $\mathrm{mg} / \mathrm{L}$ \\
\hline W-0CP & 49.07 & 2.97 & 1.92 & 0.76 & 0.01 & $\mathrm{bmdl}$ & 0.15 & 11.23 & 1.31 \\
Wainwright-10 & 10.19 & 0.64 & 1.32 & 0.62 & 0.48 & 0.01 & 20.56 & 1.48 & 0.89 \\
\hline
\end{tabular}




\section{Results and 2010 Plans}

The drilling of the Wainwright-9 and -10 delineation wells established that the Wainwright coalbed is continuous beneath the Wainwright area, that it maintains its general thickness and gas content throughout, and that it is dipping to the southeast at approximately $100 \mathrm{ft} / \mathrm{mi}$. At the end of the 2009 field season, the AREP proposed that an additional well be drilled at the abandoned Air Force radar site approximately $4 \mathrm{mi}$ southeast of Wainwright to further expand the delineation of the coal reservoir.

Although production testing confirmed that water and gas could be produced from the Wainwright coalbed, the fine sediment emanating from the uncased siltstone immediately above the coal consistently plugged the submersible pump and packed in the well, thus impeding water and gas flow from the coal reservoir. This made implementation of a meaningful extended production test virtually impossible. Testing also made clear that, barring an external heat source, water pumped through the permafrost zone to land surface would freeze in the fluid-discharge pipe. The installation of two heat-trace lines in the well through the permafrost zone effectively mitigated this problem.

By the conclusion of 2009 testing activities, it became clear that the Wainwright coalbed would need to be isolated from the uncased, overlying siltstone before a meaningful pump test could be accomplished using the W-OCP production well. During the winter of 2009-2010, NSB contracted Wood Group Production Services, a petroleum engineering firm based in Houston, Tex., to formulate and oversee implementation of a 2010 production and well-stimulation plan. Discussions were held between the USGS and Wood Group during which plans were developed for the upcoming field season. The agreed-upon plan called for the following:

1. Flushing the existing gravel, sand, and sediment from the production well to well bottom $(1,295 \mathrm{ft})$.

2. Filling the uncased portion of the well with Halliburton ${ }^{\mathrm{TM}}$ quick-set cement.

3. Drilling a 6-in. hole through the cement from the base of the production casing $(1,234 \mathrm{ft})$ to $1,260 \mathrm{ft}$.

4. Underreaming the Wainwright coalbed to a 12-in. diameter.

5. Stimulate/fracturing the Wainwright coalbed using a GasGun ${ }^{\mathrm{TM}}$ solid propellant stimulation tool.

6. Installing a surface-drive 20 -horsepower Weatherford ${ }^{\mathrm{TM}}$ progressive cavity (PC) pump better suited for sediment-laden fluid production.

7. Conducting a 2-month pump test.

In addition, the Wood Group requested that all OC ware-yard monitor wells be plugged and abandoned prior to production test startup due to reservoir interference concerns.

\section{Project Activities}

Drill-crew personnel arrived in Wainwright on June 1 and spent the next several days removing snow from the work area and preparing for the arrival of the C-130 air transport carrying the Wood Group production equipment. Equipment to be backhauled 
on the return flight to Anchorage was also prepared and packed. The C-130 arrived and was unloaded on June 6. After plugging monitor well W-OC2 per AOGCC oversight on June 10, operations were halted by the AOGCC because the fluid diverter system utilized during drilling operations had inadvertently been shipped to Anchorage on the June 6 backhaul flight. Since, by AOGCC rules, installation of the diverter was required for all well-plugging and reentry activities, drill-crew personnel had to fly to Anchorage and ship the diverter back to Wainwright. Due to the time lost, rather than abandoning the remaining monitor wells prior to production-test startup, the drill rig was repositioned over the W-OCP production well on June 16. The well was cleaned and flushed to a depth of 1,280 ft, and on June 19, cement was mixed and pumped from the bottom of the well to a depth of 1,200 ft. However, when the cement was drilled from the well the following day, it was apparent that the cement had not fully cured. The bottom of the hole was subsequently recemented with heavier cement and allowed to cure for an additional 24 hours. The cement was drilled out to $1,260 \mathrm{ft}$, and the Wainwright coalbed was underreamed from 1,242 to 1,250 ft on June 21-22. On June 23, the coalbed was fractured with the GasGun stimulation tool and the well was cleaned, flushed, and filled with the antifreeze mixture. The PC pump was set below the coal zone to a depth of 1,253 ft, the drive rods and drive head were installed (fig. 26), flow meters were hooked up, the pump was turned on, and production testing began on the afternoon of June 25. However, after several hours of high-fluid discharge and rapid well-drawdown, flow from the fluiddischarge pipe slowed to a trickle. After "stroking" the pump by lifting the drive rods and pump rotor from the pump housing and back flushing fluid through the pump, pumping continued for another few hours before again slowing to a trickle. After several more pump and back-flush cycles, the pump was raised to $1,245 \mathrm{ft}$, and the test was restarted. Although the pump ran from this depth through June 30, and small volumes of gas were produced from the production casing, the discharge fluid remained silt-laden and repeated back flushing was required to keep the pump operating. On June 30, the well was filled with antifreeze and the pump was raised inside the 6-in. casing to a depth of 1,212 $\mathrm{ft}$. The pump was operating from this depth without back flushing for several days but, the produced fluid remained silt-laden. On July 3 the PC pump bound up and tripped the control-panel circuit breaker. At that point, the well was flooded with antifreeze, and the pump was removed from the well. 


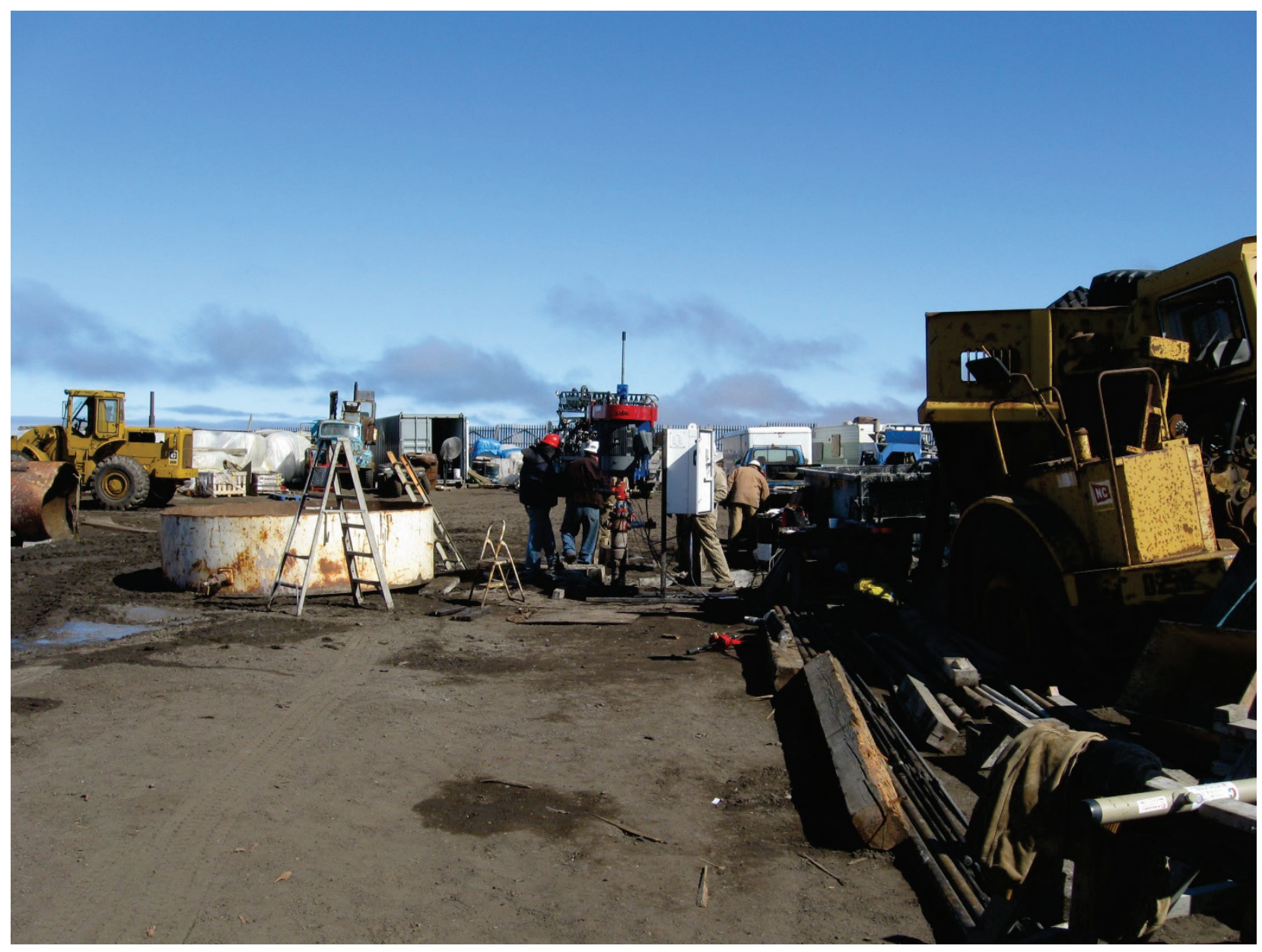

Figure 26. Surface-drive head assembly installed on W-OCP production well.

It became clear that the cement pumped into the well had failed to stabilize the uncased sediments and isolate the Wainwright coalbed as intended and that a new test plan was needed. After discussion, two options were presented to the NSB for consideration: (1) plug and abandon the W-OCP well and drill a new production well with production casing set into the top of the Wainwright coalbed or (2) clean and flush the $\mathrm{W}-\mathrm{OCP}$ well to 1,260 ft, fill the uncased portion of the well with a pea-gravel filter material, and continue production testing with the pump set above the gravel and inside the production casing. Due to time and financial constraints and after consultation with the Wood Group and other project partners, the NSB decided to clean, filter-pack, and continue testing the W-OCP well as per option 2 rather than drill a new well. As no gravel or other coarse filter material was available in Wainwright, and all available wellbore antifreeze had been used, drill-crew personnel left Wainwright on July 7 to allow for acquisition of required supplies and transport to Wainwright.

Drill-crew personnel returned to Wainwright on July 26 and cleaned, flushed, and stabilized the production well to $1,260 \mathrm{ft}$ on July 27-28. A caliper log was run below the production casing on July 29 confirming that the cement had not properly set and that the uncased portion of the well, in particular a 1-ft zone from a depth of 1,238 to 1,239 ft, had caved to a considerable diameter (fig. 27). On July 30, after placing a pea-gravel 
filter pack from 1,260 to $1,220 \mathrm{ft}$, the well was filled with antifreeze and the PC pump was set inside the casing to a depth of 1,215 ft. Production testing resumed on July 31 . Although there was a significant decrease in the amount of silt produced, the volume of produced water and gas was constant but remained low. Once production values stabilized on Aug. 2, the drilling rig was pulled off the W-OCP well and used to plug and abandon wells Wainwright-1, Wainwright-2, W-OC1, W-OC3, and W-OC4 per AOGCC guidelines and oversight. After repositioning the rig over the production well, the test was terminated on Aug. 22, and the well was filled with antifreeze. The PC pump was pulled from the well, all production equipment was removed from the site, and the W-OCP well was plugged and abandoned per AOGCC guidelines and oversight on Aug. 25. After cleaning and packing the equipment for barge shipment, drill-crew personnel left Wainwright on Aug. 28. Personnel contracted from Olgoonik Energy Services loaded the equipment on the yearly southbound barge that departed Wainwright in midSeptember and arrived in Seattle in early October. In Seattle, the equipment was transferred to highway transport vehicles and driven to Denver, arriving in late October. 


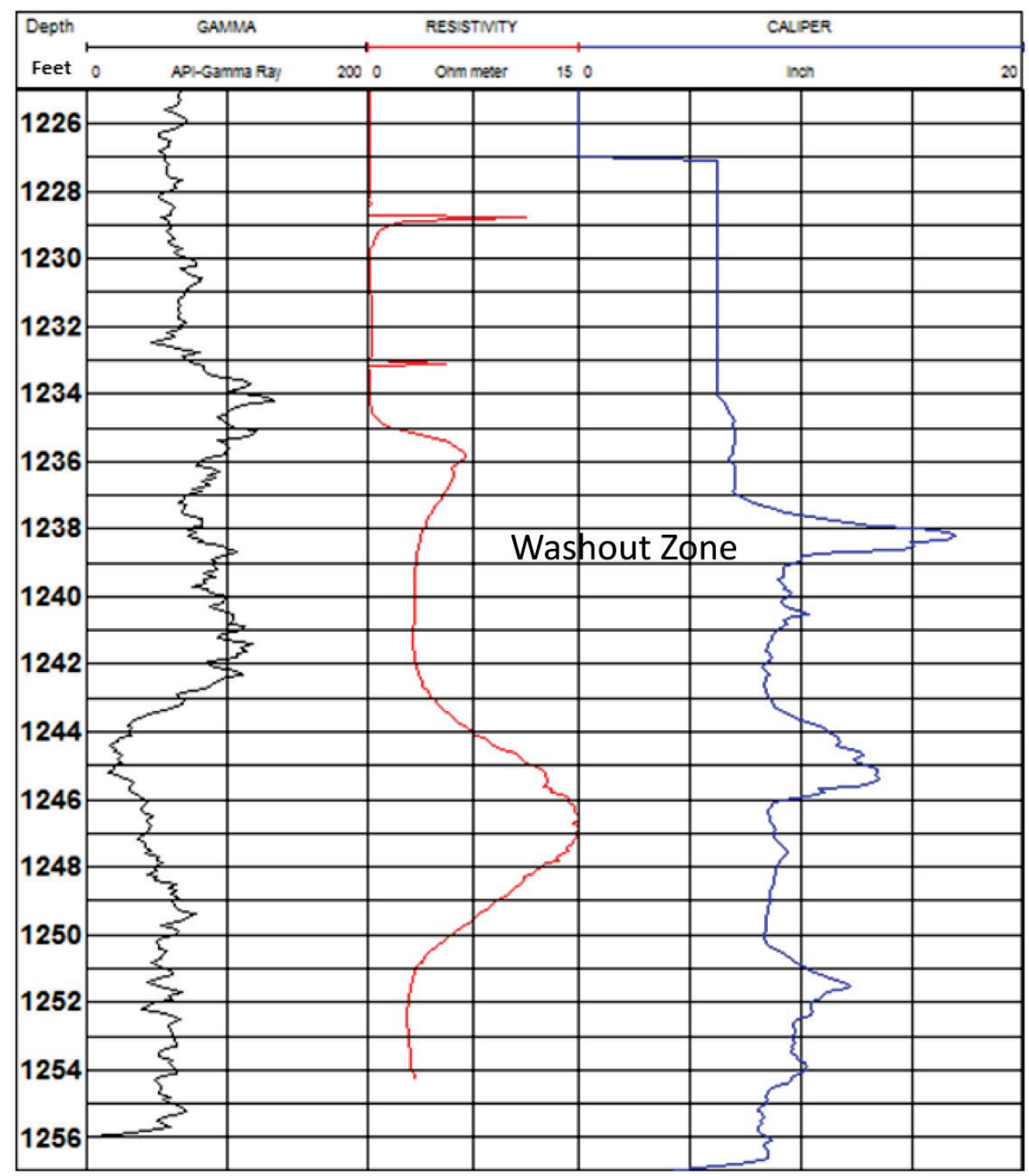

Figure 27. Caliper log collected from uncased portion of the W-OCP well on July 29, 2010.

\section{Results}

Due to the inability to stabilize the uncased portion of the $\mathrm{W}-\mathrm{OCP}$ production well and isolate the Wainwright coalbed from the overlying siltstone, the 2010 production test results were disappointingly similar to those obtained in 2009. Although a 21-day production test was conducted in which water and gas were continuously produced from the Wainwright coalbed, well-bore plugging with fine silt continued to be a major problem and produced-gas volumes were considerably less than anticipated. Although the installation of a coarse filter-pack successfully removed most sediment from the produced water, test results suggest that the filter material itself became clogged with fine sediment over time. 
All 2010 production data was collected and analyzed by Wood Group personnel. A confidential report titled "Wainwright CBNG Feasibility Study" was submitted by the Wood Group to the NSB in September 2011.

\section{Project Summary}

The Wainwright, Alaska, coalbed natural gas study discovered and delineated a substantial CBNG resource beneath Wainwright and vicinity with the potential to serve as a long-term energy supply for the community. The primary coalbed of interest, informally named the Wainwright coalbed, maintained an approximate 7.5-ft thickness throughout the study area and contained an average of $137.6 \mathrm{scf} /$ ton gas on a raw basis as desorbed from seventeen $1-\mathrm{ft}$ core samples collected from three drill locations. The eight Wainwright coalbed cores from which ash content was analyzed averaged $190.7 \mathrm{scf} /$ ton on a DAF basis. Analysis of desorbed- and produced-gas samples indicate that the gas contained within the bed is $>98$ percent methane. Isotherm analysis of coal core samples, though conflicting, suggests that the coalbed is saturated with respect to methane gas and that gas desorption from the coal could start as soon as reservoir pressure is reduced. The top of the Wainwright coalbed lies in the subpermafrost at depths ranging from 1,167 to $1,300 \mathrm{ft}$ in the three drilled wells and is dipping to the southeast at approximately 100 $\mathrm{ft} / \mathrm{mi}$. The horizontal bedding and a general lack of structural features in the subsurface beneath Wainwright suggest that the coalbed likely deepens and extends for an unknown but potentially considerable distance in a southeasterly direction. A well drilled at the abandoned Air Force radar site approximately 4 mi southeast of the community would better define the Wainwright coalbed geometry and substantially increase the delineated potential resource area.

Given the 7.5-ft-bed thickness, the average raw desorption value, and a subbituminous coal specific gravity of $1.4 \mathrm{~g} / \mathrm{cm}^{3}$, the volume of methane gas contained within the Wainwright coalbed calculates to $1.26 \mathrm{bscf} / \mathrm{mi}^{2}$ of the bed. Based on an energy balance of 1,000 standard cubic feet of natural gas equaling 7.2 gal of diesel fuel, replacing the annual community usage of approximately 1,000,000 gal of diesel fuel would require 0.14 bscf of natural gas. Assuming that somewhere between 25 and 75 percent of the in-place gas could be produced, the gas contained within each square mile of the Wainwright coalbed represents between a 2- and 6-year energy supply for the community. Given the apparent continuity of the coalbed and the expansive area surrounding Wainwright, the Wainwright coalbed could, given the proper production techniques, serve as a clean, local community power source for the foreseeable future.

Production testing of the Wainwright coalbed proved challenging. The problems related to producing gas and water through 1,000 ft of permafrost were numerous and effectively addressing these issues was difficult, expensive, and time consuming. The potential for borehole freeze up and subsequent loss of the production well and equipment was a constant concern. To prevent freezing in the well-bore annulus, a propylene glycol/water antifreeze mixture was the only fluid maintained in the top 1,000 $\mathrm{ft}$ of the annular area for appreciable lengths of time. During testing, attempts were made to keep the annular-fluid level below the permafrost zone where freezing was not an issue. However, when problems were encountered and pumping was suspended or interrupted for extended periods, the annular area had to be flooded with the antifreeze mixture to prevent formation water from rising to its equilibrium level and freezing in the 
well. Given the number of interruptions that occurred during the two testing seasons, the amount of propylene glycol used during the project was considerable. The freezing of fluid in the water-discharge pipe was a problem solved by attaching two 1,100-ft lengths of heat trace to the pipe. Although the hydrostatic pressure exerted on the down-hole end of the trace lines resulted in several electrical shorts, line-end protection methods were developed that effectively mitigated the problem. Although most permafrost-related production issues were successfully addressed for this project, effective production of the resource would likely require that wells be engineered so that produced fluids could be disposed directly into suitable subpermafrost zones rather than pumping them to land surface. Although potential fluid-disposal zones were not specifically identified or evaluated during the study, the $2,450-\mathrm{ft} \mathrm{W}-\mathrm{OC} 1$ well penetrated numerous sand bodies beneath the Wainwright coalbed with the potential to serve this purpose. A thick subpermafrost sandstone penetrated approximately $200 \mathrm{ft}$ above the Wainwright coalbed in all drilled wells may have fluid-disposal potential as well.

Although small quantities of gas and water were produced on a continuous basis, produced volumes did not increase over time as anticipated and commercial quantities of gas were not produced. The lack of production appeared to primarily relate to the method in which the production well was completed. In an effort to retain the full coalbed thickness for testing and to protect the coal from possible cement damage, production casing was set and cemented $8 \mathrm{ft}$ above the coalbed rather than directly into the bed itself. Because the uncased sediments between the base of the casing and the top of the coalbed proved less stable than originally evaluated, the sediment ultimately began to cave and slough when exposed to formation fluid over an extended period of time. The resulting fine-grained sediment load continually plugged the production pump and settled in the uncased portion of the well, greatly impeding water and gas flow from the coal. Efforts to stabilize these sediments and isolate the coalbed for production testing proved unsuccessful and a definitive, uncompromised production test could therefore not be conducted. This leaves the true production potential of the resource in question. However, given the relatively high volumes of gas contained within the Wainwright coalbed and the relatively low volumes of gas required to power the community, it seems likely that, with proper well-field design and by utilizing currently available drilling and reservoir stimulation techniques, this CBNG resource could be developed as a long-term alternative energy source for Wainwright.

\section{Acknowledgments}

The Wainwright CBNG exploration project was a collaborative effort that could not have been accomplished without the participation and assistance of numerous individuals and project partners. Special thanks go to Marvin Olsen, Matthew Dunn, Bernadette Suvlu, and the entire North Slope Borough for their funding and support as well as to Teresa Imm of the Arctic Slope Regional Corporation for her backing, assistance, and insight. The assistance of Lucille Mayer and other individuals from the Olgoonik Corporation and Olgoonik Energy Services helped keep the project moving forward. A special thanks goes to Robert Fisk of the Alaska Bureau of Land Management for his backing and support as well as to Greg Noble, Beth Maclean, and others from within the Alaska BLM for their financial and technical assistance. Invaluable scientific and technical support was provided by numerous current and former employees of the 
USGS including Stephen Roberts, Edwin Weeks, Charles Barker, Peter Warwick, Ralph Wilcox, Margo Corum, Jon Haacke, John Earle, Greg Clark, Steve Harris, and Barbara Corland. The project could not have been accomplished without the extraordinary efforts put forth by Jeffrey Eman and the entire USGS research drilling crew. Lastly, a heartfelt thank you goes to the citizens of Wainwright whose warmth and friendship made project members feel welcome throughout.

\section{References Cited}

American Society for Testing and Materials (ASTM), 1981, Annual book of ASTM standards; part 26, gaseous fuels; coal and coke; atmosphere analysis: Philadelphia, Pa., American Society for Testing and Materials, p. 181-399.

American Society for Testing and Materials (ASTM), 2011, Standard practice for determination of gas content of coal—Direct desorption method: Philadelphia, Pa., American Society for Testing and Materials, $12 \mathrm{p}$.

Ahlbrandt, T.S., Huffman, A.C., Fox, J.E., and Pasternack, I., 1979, Depositional framework and reservoir-quality studies of selected Nanushuk Group outcrops, North Slope, Alaska, in Ahlbrandt, T.S., ed., Preliminary geologic, petrologic, and paleontologic results of the study of Nanushuk Group rocks, North Slope, Alaska: U.S. Geological Survey Circular 794, p. 14-31.

Barker, C.E., and Dallegge, T.A., 2005, Zero-headspace coal-core gas desorption canister, revised desorption data analysis spreadsheets and a dry canister heating system: U.S. Geological Survey Open-File Report 2005-1177, 8 p.

Barker, C.E., Dallegge, T.A., and Clark, A.C., 2002, USGS coal desorption equipment and a spreadsheet for analysis of lost and total gas from canister desorption measurements: U.S. Geological Survey Open-File Report 2002-496, 13 p. plus spreadsheet.

Callahan, J.E., and Sloan, E.G., 1978, Preliminary report on analyses of Cretaceous coals from northwestern Alaska: U.S. Geological Survey Open-File Report 78-319, 29 p., $1 \mathrm{pl}$.

Chapman R.M., and Sable, E.G., 1960, Geology of the Utukok-Corwin region, northwestern Alaska: U.S. Geological Survey Professional Paper 303-C, p. 47-174.

Clark, A., Barker, C.E., and Weeks, E.P., 2009, Drilling and testing the DOI-04-1A coalbed methane well, Fort Yukon, Alaska: U.S. Geological Survey Open-File Report 2009-1064, 69 p.

Clark, A.C., Roberts, S.B., Warwick, P.D., 2010, Geologic cross section, gas desorption, and other data from four wells drilled for Alaska Rural Energy Project, Wainwright, Alaska, Coalbed Methane Project, 2007-2009: U.S. Geological Survey Open-File Report 2010-1210, 1 pl.

Diamond, W.P., and Levine, J.R., 1981, Direct method determination of the gas content of coal-Procedures and results: U.S. Bureau of Mines Report of Investigations 8515, $36 \mathrm{p}$.

Diamond, W.P., and Schatzel, S.J., 1998, Measuring the gas content of coal-A review, in Flores, R.M., ed., Coalbed methane-From coal-mine outbursts to a gas resource: International Journal of Coal Geology, v. 35, p. 311-331.

Flores, R.M., Stricker, G.D., and Kinney, S.A., 2004, Alaska coal geology, resources, and coalbed methane potential: U.S. Geological Survey Digital Data Series 77, 125 p. 
Mavor, M., and Nelson, C.R., 1997, Coalbed reservoir gas-in-place analysis: Gas Research Institute Report no. GRI-97/0263, 134 p.

Moore, T.E., Wallace, W.K., Bird, K.J., Karl, S.M., Mull, C.G., and Dillon, J.T., 1994, Geology of northern Alaska, in Plafker, George, and Berg, H.C., eds., The geology of Alaska: Geological Society of America, Geology of North America, v. G-1, p. 49-140.

Molenaar, C.M., 1985, Subsurface correlations and depositional history of Nanushuk Group and related strata, North Slope, Alaska, in Huffman, A.C., Jr., ed., Geology of the Nanushuk Group and related rocks, North Slope, Alaska: U.S. Geological Survey Bulletin 1614, p. 37-59.

Mull, C.G., Houseknecht, D.W., and Bird, K.J., 2003, Revised Cretaceous and Tertiary stratigraphic nomenclature in the Colville Basin, northern Alaska: U.S. Geological Survey Professional Paper 1673, 22 p.

Roberts, S.B., Bird, K.J., Charpentier, R.R., Cook, T.A., Houseknecht, D.W., Klett, T.R., Pollastro, R.M., and Schenk, C.J., 2008, Executive summary-Geologic assessment of undiscovered, technically recoverable coalbed-gas resources in Cretaceous and Tertiary rocks, North Slope and adjacent State waters, Alaska: U.S. Geological Survey Digital Data Series DDS-69-S, chap. 1, 4 p.

Roberts, S.B., 2008, Geologic assessment of undiscovered, technically recoverable coalbed-gas resources in Cretaceous and Tertiary rocks, North Slope and adjacent State waters, Alaska, in Roberts, S.B., comp., Geologic assessment of undiscovered, technically recoverable coalbed-gas resources in Cretaceous and Tertiary rocks, North Slope and adjacent State waters, Alaska: U.S. Geological Survey Digital Data Series DDS-69-S, chap. 2, 95 p., 2 pls.

Sable, E.G., and Stricker, G.D., 1987, Coal in the National Petroleum Reserve in Alaska (NPRA) - Framework geology and resources, in Tailleur, I.L., and Weimer, Paul, eds., Alaskan North Slope geology: Bakersfield, Pacific Section, Society of Economic Paleontologists and Mineralogists SEPM Book No. 50, p. 195-215.

Tyler, Roger, Scott, A.R., and Clough, J.G., 2000, Coalbed methane potential and exploration targets for rural Alaskan communities: Alaska Division of Geological and Geophysical Surveys Preliminary Interpretive Report 2000-2, 177 p.

Van Vost, W.A., 2003, Geochemical signature of formation waters associated with coalbed methane: American Association of Petroleum Geologists Bulletin, v. 87, p. 667-676.

Wahrhaftig, Clyde, Bartsch-Winkler, Susan, and Stricker, G.D., 1994, Coal in Alaska, in Plafker, George, and Berg, H.C., eds., The geology of Alaska: Geological Society of America, The Geology of North America, v. G-1, p. 937-978.

Whiticar, M.J., 1999, Carbon and hydrogen isotope systematics of bacterial formation and oxidation of methane: Chemical Geology, v. 161, p. 291-314. 